\title{
University of Cologne
}

\section{Spatio-temporal analysis of architecture and growth in bacterial colonies}

\author{
-Inaugural-Dissertation- \\ zur Erlangung des Doktorgrades \\ der Mathematisch-Naturwissenschaftlichen Fakultät \\ der Universität zu Köln
}

vorgelegt von

Anton Welker

aus Omsk 
Berichterstatter:

(Gutachter)
Prof. Dr. Berenike Maier

Prof. Dr. Tobias Bollenbach

Tag der mündlichen Prüfung: 31.08.2021 




\section{Abbreviations}

\begin{tabular}{ll} 
CCW & Counterclockwise \\
CLSM & Confocal laser scanning microscopy \\
CW & Centre of mass \\
OD & Clockwise \\
PI & Optical density \\
RDF & Propidium iodide \\
RNA & Radial distribution function \\
MIC & Ribonucleic acid \\
SNR & Minimal inhibitory concentration \\
ppGpp & Signal to noise ratio \\
pppGpp & Tetraphosphate guanosine \\
T4P & Pentaphospate guanosine \\
wt & Type 4 pilus \\
\hline
\end{tabular}




\section{Contents}

Abbreviations ।

1 Abstract 1

2 Zusammenfassung 3

3 Introduction $\quad 5$

3.1 Bacterial surface motility . . . . . . . . . . . 7

3.1.1 Bacterial surface sensing and attachment . . . . . . . 7

3.1 .2 Twitching motility $\ldots \ldots \ldots \ldots \ldots \ldots$

3.1 .3 T4P machinery $\ldots \ldots \ldots \ldots \ldots \ldots \ldots$

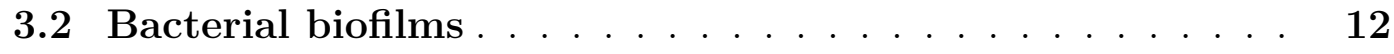

3.2 .1 Development of biofilms . . . . . . . . . . . 12

3.2 .2 Structure and dynamics of biofilms _ . . . . . . . . 13

3.2.3 Phenotypic heterogeneity and stress response in biofilms . 17

3.3 Cell and particle tracking methods . . . . . . . . . . 21

3.3 .1 Image segmentation . . . . . . . . . . . . . . . 21

3.3 .2 Particle localization . . . . . . . . . . . . . . . . 22

3.3 .3 Particle tracking . . . . . . . . . . . . . . . 23

3.3.4 Alternative particle tracking tools . . . . . . . . . . 24

4 Aims of this study $\quad 25$

5 Material and Methods 27

5.1 Cultivation and Growth Media . . . . . . . . . . . 27 
5.2 Bacterial Strains . . . . . . . . . . . . . . . . . . . . . 28

5.3 Choosing the experimental design . . . . . . . . . . . . . 31

5.3.1 Experiments to study the colony structure . . . . . . . 31

5.3.2 Experiments to study the spatio-temporal growth dynamics 32

5.4 General tracking procedures . . . . . . . . . . . . . . 34

5.4.1 Procedures for image segmentation . . . . . . . . . . . 34

5.4.2 Segmentation of images acquired by spinning disk confocal microscopy . . . . . . . . . . . . . . . 35

5.4.3 Procedures for localizing individual cells . . . . . . . . 38

5.4.4 Procedures for tracking cells . . . . . . . . . . . . . . 39

5.5 Effects of parameter variation on the RDF . . . . . . . 40

5.6 Implementation of particle tracking . . . . . . . . . . . . . 42

5.7 Limitations in particle localization and tracking . . . . . . 44

5.7.1 Pixel-biasing and SNR influence particle localization . . . 44

5.7.2 Motility and density influence particle tracking . . . . . . . 45

5.8 Data analysis . . . . . . . . . . . . . . . . . . 48

5.8.1 Characterization of colony structure . . . . . . . . . 48

5.8.2 Growth rate determination . . . . . . . . . . . . 49

5.8.3 Determination of fraction of dead cells . . . . . . . . 52

6 Results 53

6.1 Structural properties of gonococcal colonies . . . . . . . . . 54

6.1.1 Gonococcal colonies show liquid-like order . . . . . . . . 54

6.1.2 Motor activity accelerates local-ordering . . . . . . . . 54

6.1.3 Gonococcal colonies show defects in their structure . . . . 60

6.2 Dynamics and growth within bacterial colonies . . . . . . . 62

6.2.1 Colony growth shows deviations from exponential growth after two hours . . . . . . . . . . . . 62

6.2.2 Lineage tracking within gonococcal colonies reveals development of growth profiles . . . . . . . . . . . . . . 63

6.2.3 Increasing nutrient supply does not prevent growth inhibition at the center of the colony . . . . . . . . . . 72 
6.2.4 Lack of stringent response accelerates heterogeneity in spatiotemporal growth dynamics . . . . . . . . . . . . 74

6.2.5 Effect of antibiotic treatment on growth and death dynamics of gonococcal colonies . . . . . . . . . . . . . 77

7 Discussion $\quad 80$

7.1 Gonococcal colonies are reminiscent of non-living colloidal systems . . . . . . . . . . . . . . . . . . . . 80

7.2 Deletion of the T4P retraction motor severely slows down the process of local ordering . . . . . . . . . . . . . . 81

7.3 Determining growth rates with spatial and temporal resolution in spherical colonies . . . . . . . . . . . .

7.4 Growth rate gradients emerge within a few generations and stringent response retards the development of spatially heterogeneous growth rates . . . . . . . . . . . . . 84

7.5 Azithromycin is more efficient against fast growing cells . 86

8 Outlook $\quad 88$

9 Appendix $\quad 92$

9.1 Particle tracking software . . . . . . . . . . . . . . 92

$\begin{array}{lr}\text { List of Figures } & 94\end{array}$

$\begin{array}{ll}\text { List of Tables } & 96\end{array}$

$\begin{array}{ll}\text { Bibliography } & 97\end{array}$ 


\section{Abstract}

Most microorganisms prefer to live in surface associated communities called biofilms, where their lifestyle differs considerably compared to their planktonic counterpart. The cell shape, as well as physical interactions determine the structure of bacterial biofilms. Due to cell growth, the size of the biofilm increases with time and the structure changes during biofilm maturation. This work aims at characterizing the structure and growth dynamics of dense colonies formed by gonococci.

The first part of this thesis focuses on the characterization of the spatial structure of gonococcal colonies. Image analysis tools were developed that allowed determining the positions of single cells within the spherical colonies. Using the position data, the radial distribution function (RDF) was calculated. The RDF showed short-ranged order but not long range order, reminiscent of liquids. Neisseria gonorrhoeae interact via their type 4 pilus (T4P) with surfaces and cells. T4P$\mathrm{T} 4 \mathrm{P}$ binding between adjacent cells generates attractive force that controls colony formation. We investigated the effect of T4P retraction of the local and mesoscopic structure of gonococcal colonies using strains with varying T4P retraction phenotypes. Reducing speed and frequency of T4P retraction reduced cell density and increased order in $6 \mathrm{~h}$ old colonies. Deleting T4P retraction results in loss of local order. After $24 \mathrm{~h}$, density and local order increase for all strains, and larger holes inside the structures of gonococcal colonies emerged, which were independent of T4P motor activity. In conclusion, we show that gonococcal T4P active force generation is not necessary for development of local order, but it accelerates the process of achieving higher densities and local ordering inside spherical colonies. 
In the second part of this thesis, we developed methods for measuring growth rates of colony-bound bacteria with spatial and temporal resolution. Growing gonococci generated a radial velocity field inside colonies that pointed from the centre of mass (COM) of colonies to its periphery. Close to the colony centres, velocities were minimal and increased towards the periphery of colonies. We showed that by characterizing the velocity field within the colony, the local growth rates can be measured. Independently, growth rates were determined by counting the offspring of single fluorescent cells that were distributed homogeneously inside the colonies. Both methods complement each other, because they have different advantages and disadvantages. Unexpectedly, heterogeneous growth profiles inside small gonococcal colonies emerged after $2 \mathrm{~h}$ of growth. To assess the hypothesis that nutrient limitation causes growth heterogeneity, we optimized the nutrient supply with a higher flow rate. Even though gonococcal growth improved slightly, growth profiles were still heterogeneous, indicating different limitations like mechanical constraints. Surprisingly, colonies that could not activate the stringent response developed heterogeneity in spatial and temporal growth even earlier. We suggest that stringent response is important for gonococcal biofilm maturation. Finally, the effect of azithromycin treatment on colony growth dynamics was investigated. We observed that after two generations times, growth rates dropped to low values throughout the colony indicating that azithromycin diffuses quickly through the whole colony and effects the majority of cells. In summary, we established tools for characterizing growth and death within dense spherical colonies at spatial and temporal resolution. This method will be useful to study the mechanisms of development of heterogeneity inside gonococcal colonies and their response to environmental changes like antimicrobial treatment. 


\section{Zusammenfassung}

Die meisten Mikroorganismen bevorzugen es in an Oberflächen gebundenen Gemeinschaften zu leben, welche als Biofilme bekannt sind. Dabei unterscheidet sich ihr Lebensstil wesentlich von planktonischen Zellen. Die Zellform und die physikalischen Wechselwirkungen bestimmen die Struktur des Biofilms. Durch Zellwachstum nimmt die Größe des Biofilms mit der Zeit zu und die Struktur des Biofilms ändert sich dabei parallel zum Entwicklungsstand des Biofilms. Diese Arbeit beschäftigt sich mit der Struktur und Wachstumsdynamik von dichten Kolonien, welche von kugelförmigen Zellen geformt worden sind.

Der erste Teil dieser Arbeit beschäftigt sich mit der Charakterisierung der räumlichen Struktur von durch Gonokokken geformten Kolonien. Programme zur Bildauswertung wurden entwickelt, welche die Bestimmung der räumlichen Position einzelner Zellen in den Kolonien ermöglicht. Mit den Positionskoordinaten wurde die radiale Verteilungsfunktion berechnet. In der radialen Verteilungsfunktion zeigt sich eine kurzreichweitige Ordnung der Zellen, die an die Ordnung von Molekülen in einer Flüssigkeit erinnert. Neisseria gonorrhoeae wechselwirkt mit dem Typ 4 Pilus (T4P) mit Oberflächen und anderen Zellen. T4P-T4P Bindungen zwischen benachbarten Zellen erzeugt eine anziehende Kraft, welche die Kolonie-Entstehung beeinflusst. Wir haben den Effekt der T4P Retraktion auf die lokale und mesoskopische Struktur von Kolonien untersucht, wobei wir bakterielle Stämme mit unterschiedlichen T4P Retraktionseigenschaften verwendet haben. Eine reduzierte Geschwindigkeit und Häufigkeit der T4P Retraktion bewirkte eine geringere Zelldichte und eine höhere lokale Ordnung von 6 Stunden alten Kolonien. Kolonien, deren Zellen ihre T4P nicht retrahieren konnten, wiesen nach 6 Stunden keine lokale Ordnung auf. Nach 24 Stunden, wiesen jedoch alle verwendeten Stämme eine höhere Zelldichte und lokale Ordnung in den jeweiligen Kolonien 
auf. Außerdem bildeten sich Löcher in allen Kolonien, welche unabhängig von der T4P Motor Aktivität waren. Zusammenfassend, konnten wir zeigen, dass T4P Retraktion zur Entstehung von lokaler Ordnung nicht erforderlich sind, sie aber den Prozess zur Entwicklung höherer Zelldichten und lokaler Ordnung beschleunigen.

Im zweiten Teil entwickelten wir Methoden zur Bestimmung von Wachstumraten für Kolonie-gebundene Bakterien mit räumlicher und zeitlicher Auslösung. Wachsende Gonokokken entwickelten ein radiales Geschwindigkeitsfeld, welches vom Kolonie-Zentrum zur dessen Peripherie zeigte. In der Nähe des Kolonie-Zentrums waren die Geschwindigkeiten einzelner Zellen minimal and nahmen Richtung Peripherie zu. Wir zeigten, dass lokale Wachstumsraten durch Charakterisierung des Geschwindigkeitsfeldes bestimmt werden können. Unabhängig dazu, wurden Wachstumsraten durch das Zählen der Nachkommen von einzelnen fluoreszierenden Zellen bestimmt, welche innerhalb der Kolonie homogen verteilt waren. Beide verwendeten Methoden ergänzen sich, weil sie verschieden Vor- und Nachteile aufweisen. Unerwarteterweise konnten wir heterogene Wachstumsraten in kleinen Kolonien nach 2 Stunden Wachstumszeit bestimmen. Um die Hypothese zu belegen, dass heterogenen Wachstumsraten durch Nährstoffmangel hervorgerufen werden, optimierten wir die Nährstoffversorgung Erhöhung der Zufuhr von Nährstoffen. Obwohl sich das Wachstum verbesserte, waren die Wachstumsraten immer noch heterogen verteilt, sodass wir andere limitierende Faktoren wir mechanische Einschränkungen vermuten. Überraschenderweise, zeigten Kolonien dessen Zellen keine ,Stringent'Response aktivieren können, eine frühzeitige Entstehung von heterogenen Wachstumsraten. Daher vermuten wir, dass die ,Stringent'Response ein wichtiger Bestandteil der Biofilm-Entwicklung ist. Zusammenfassend, entwickelten wir Methoden zur Charakterisierung von Wachstum und Tod in dichten Kolonien mit räumlicher und zeitlicher Auflösung. Diese Methoden können verwendet werden um die Entstehung von Heterogenität in Kolonien und deren Reaktion auf wechseln Umweltbedingungen wie Antibiotika Behandlung zu untersuchen. 


\section{Introduction}

The dominant form of bacterial life was reported to be in in a surface associated biofilm, where many bacteria are organized in close proximity and interact mechanically [1]. Organization into a biofilm gives rise to different physiological behaviors $[2,3]$ and a higher stress resistance $[4,5,6,7,8]$, which cannot be understood by studying planktonic bacteria $[9,10]$.

Recently, laser scanning microscopy (CLSM) has been used to study the architecture of fixed bacterial colonies. Using particle tracking tools from colloidal physics, three dimensional positions of individual spherical cells inside colonies were determined with sub-pixel accuracy. The bacterial structure was analysed by the cell density and the radial distribution function (RDF) that gives the probability of finding bacteria at a certain distance [11]. This work revealed short-ranged order inside colonies and reported that the environmental stress influences the colony structure considerably. Furthermore, it was shown that rod-shaped Vibrio cholerae forms dome-shaped three dimensional colonies with a local liquid-crystalline order [12]. Its biofilm structure also responded to environmental conditions like starvation, shear flow and osmotic stress $[12,13]$. During biofilm development, bacteria employ various mechanical interaction mechanisms that generate attractive and repulsive forces by using for example extracellular appendages like type 4 pili (T4P) or secretion of extracellular matrix components [1]. Still, little is known about how physical interactions determine the structure of bacterial biofilms. In this study, the effect of T4P motor activity on the colony structure is investigated. 
Availability of new fluorescent proteins like superfolder GFP and progress in design of confocal microscopes allow to study the dynamical process of biofilm development with cellular resolution. Following single cell lineages inside biofilms is a difficult task due to the small size of cells $(\approx 1 \mu \mathrm{m})$ and the limited time resolution of approximately $10 \mathrm{~min}$ due to phototoxicity of the laser light. However, cell velocities and growth rates were successfully determined for $V$. cholerae biofilms. Surprisingly, growth rates were homogeneous in time and position, and velocities increased slightly for larger biofilms [12]. The used image segmentation and particle tracking algorithms are specialized on characterization of rod-shaped cells inside biofilms with a low density. So far, information about growth rates of spherical-shaped cells inside dense colonies are lacking. In this study, we develop novel methods to characterize growth dynamics inside gonococcal colonies.

In the first section of this chapter, we focus on the interaction of single cells with surfaces. In particular, we describe surface sensing and motility of cells, and introduce the T4P system. In the second section, bacterial biofilms are described in detail. In the last section, we introduce important concepts of image segmentation and particle tracking that can be useful to study bacterial biofilms. 


\subsection{Bacterial surface motility}

Due to their small size bacteria live in an environment, where viscous forces dominate the cell movement and inertial forces are negligible. Consequently, the velocity of cells depends mostly on forces that are applied at the moment and not on forces that have been applied previously. In order to be supplied with nutrients, bacteria do not have to be motile, because the transport of food is locally controlled by diffusion. However, to find places with more or better nutrients, bacteria have to outrun diffusion [14].

To this end, bacteria have evolved different molecular motors that enable them to move through fluids and on surfaces. In particular, flagella enable bacterial swimming. They are long filaments that emerge from the cell body and are powered by the flagella motor. This rotary motor converts mechanical rotation into displacement and spins clockwise (CW) or counterclockwise (CCW) [15]. While CCW rotation pushes the cell forward (run), CW rotation causes the cell to change its course (tumble) [16]. The alternation of runs and tumbles allows movement along nutrient gradients [14].

In the following sections, surface motility, which requires bacterial surface attachment, will be introduced.

\subsubsection{Bacterial surface sensing and attachment}

To attach to surfaces for surface motility and colonization, bacteria evolved various strategies like employing fimbriae, T4P or secreting extracellular polymeric substances (EPS) [17]. During the first surface contact, bacteria are able to use appendages like flagella and T4P to sense surfaces. When flagella attach to a surface, rotation of the flagella motor is obstructed and the load on the motor increases. The difference in the load on the motor causes structural changes of the stator complex, which can be seen as a mechanosensory property of the flagella motor $[18,19]$.

In contrast, T4P are typically bound to surfaces where the first contact mediates surface sensing. T4P are polymeric filaments that emerge from the cell body and 
mediate surface attachment [20]. This length of the pilus filament is dynamic. It elongates by polymerization and retracts by depolymerization [21]. Looking at the intracellular level, studies of Pseudomas aerugnisoa indicate that surface sensing via pilus occurs during pilus retraction through both, sensing of pilus tension and detection of depolymerized pilins, which has been reincooperated inside the membrane after retraction [22, 23]. Studies of Caulobacter crescentus revealed that cycles of pilus elongation and retraction cease after the first surface contact. Simultaneously the synthesis of an adhesive holdfast is promoted, starting the transition to an irreversible surface attachment [24]. Interestingly, surface sensing is reported to be a heterogeneous process, allowing bacteria to attach and detach multiple times from surfaces before they stimulate cell differentiation and attach irreversible by expressing EPS [25, 26].

\subsubsection{Twitching motility}

After surface attachment, bacteria can coordinate surface motility by a twodimensional tug-of-war mechanism. In particular, cycles of pilus elongations, binding and retraction enable cell displacement to the direction, where the pilus is bound [27]. Forces are shared when multiple attached pili retract at the same time, causing biased movement to the direction where the majority of pili are attached or this highest force is applied (Fig. 3.1). While many species are able to interact with their environment via different kinds of cell appendages, $N$. gonorrhoeae produces mainly T4P to mechanically interact with surfaces or other cells [27]. In contrast to the rod-shaped bacterium $P$. aeruginosa, who distributes pili at one of its poles [28, 29], spherical shaped bacteria like $N$. gonorrhoeae distribute pili homogeneously on their cell surface [30], leading to a random walk on longer time scales [31]. On shorter time scales, experimental and theoretical approaches show that two-dimensional tug-of-war mechanism mediate mediate bacterial persistent movement. This directional memory increases the surface area that can e explored by cells and facilitates bacterial colonization [21]. 
a

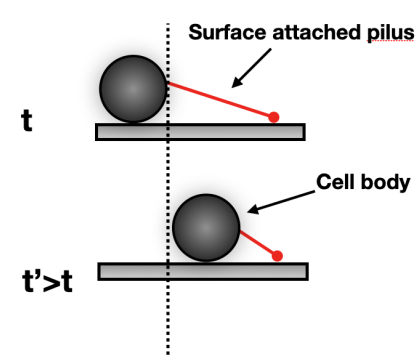

b

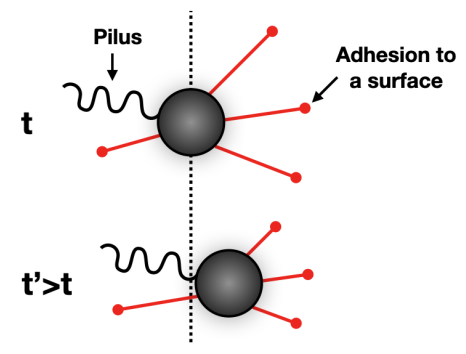

Figure 3.1: Scheme of surface motility driven by T4P. Multiple pili cooperate by a tug-of-war mechanism to induce surface movement.

\subsubsection{T4P machinery}

T4P are dynamical extracellular filaments that many bacterial species use for twitching motility [32], DNA uptake [33, 34] and microcolony formation [35, 36, $37,38]$. In this section, we will focus on the T4P architecture of $N$. gonorrhoeae and its role in microcolony formation.

\section{T4P architecture}

The main building blocks of the T4P machinery are the pilus fiber, an inner membrane platform protein, an outer membrane secretin channel and two ATPases that power pilus elongation and retraction [39], (Fig. 3.2). The pilus fiber is a long elastic polymer with a width of 5-8 nm and a length of several micrometers [40, 41]. The polymer mainly consists of pilins, whose assembly starts at the inner membrane platform protein. Through the secretin channel inside the outer membrane, the pilus can grow to the outside of the cell [42]. The pilus length is dynamical and pilus elongation and retraction is powered by two different hexameric ATPases that bind to the inner membrane platform protein [43]. 


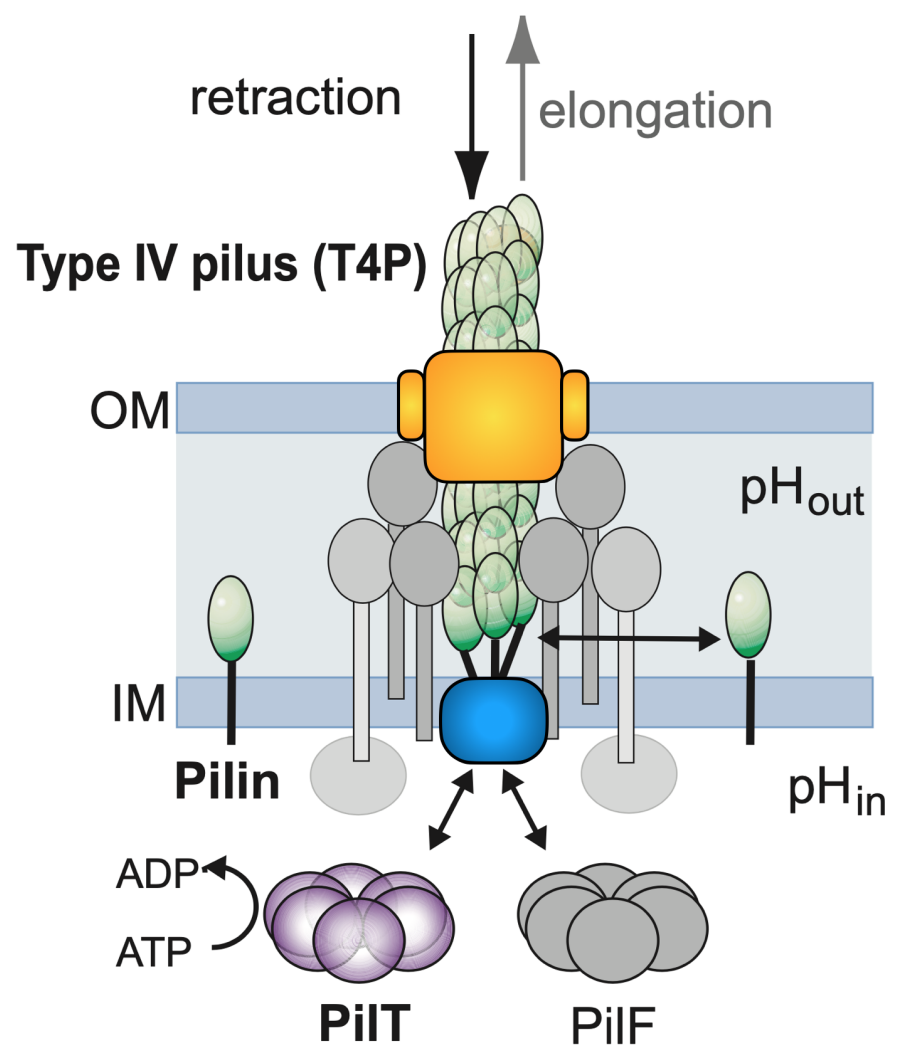

Figure 3.2: Molecular model of T4P. T4P consists of a polymeric fiber that is built mainly of the major pilins (green). It is anchored at platform protein (blue) located in the inner membrane (IM) and exits the surface of the cell though a secretin channel (orange) in the outer membrane (OM). The length of the pilus fiber is dynamic, where PilF elongates the fiber by polymerization and PilT causes retraction by depolymerization. Image adapted from [44] and reproduced with permission.

\section{T4P dynamics}

The pilus fiber actively elongate and retract, where PilF and PilT most likely add or remove pilins from the fibre. The current model states that they generate conformational changes in the platform complex which somehow supports assembly and disassembly. Possibly the platform complex rotates in opposite directions when PilT or PilF are bound [40]. The T4P machinery is one of the strongest molecular motors $[45,46]$ that generates forces up to $150 \mathrm{pN}$ during pilus retraction 
$[45,33,46,47,48]$. In order to generate those forces, the retraction ATPase PilT is essential. PilT converts chemical energy from ATP binding and hydrolysis to mechanical energy [49]. Deletion of PilT allows pilus biogenesis and colony formation, but active force generation is inhibited [27]. Even though pilus retraction was reported in the absence of PilT, the measured forces were only the order of $5 \mathrm{pN}$.

T4P activity can be fine-tuned by co-expressing functional and non-functional pilT. When non-functional PilT is produced inside the cell, hexamers of functional and non-functional PilT are formed that exert a negative effect on T4P motor activity. While retraction velocity, the frequencies of pilus retraction and T4P-T4P bond rupture decreased, the frequency for pilus elongation increased [44]. The respective mutant strains will be used in chapter 6.1.2 to investigate the effects of T4P dynamics on the architecture of gonococcal colonies. Besides manipulating T4P motor activity, T4P-T4P interaction can be influenced by altering pilus density [50], posttranslational modifications [51] or environmental changes like oxygen-concentrations [52] and proton motive force (PMF) [53]. 


\subsection{Bacterial biofilms}

Bacteria are reported to be the most successful form of life on earth with respect to biomass and diversity of colonized habitats [3]. The key to this success is phenotypical plasticity [54] that allows rapid adaptation to environmental changes. Individual motile bacteria can attach to surfaces and aggregate in large communities, called biofilms [55,3]. Typically, biofilms are embedded in an extracellular matrix that consists of extracellular polymeric substances (EPS) like extracellular DNA, polysaccharide or proteins [17]. The biofilm matrix immobilizes cells, provides mechanical stability, facilitates cell-cell interactions by keeping cells in close proximity and increases resilience against environmental stress $[17,56,57]$. Within biofilms, the production of extracellular matrix can be inhomogeneous distributed and for some species solely specialized cells contribute to the production [58].

\subsubsection{Development of biofilms}

Experimental approaches using microscopy enabled visualization of biofilm development. These observations suggest that biofilms develop in four distinct stages: (i) bacterial attachment to a surface, (ii) formation of micro-colonies, (iii) maturation of micro-colonies to colonies/ biofilms and (iv) biofilm dispersal (Fig. 3.3). Between each stage, the expression level of detectable proteins changes considerably [59].

The transition from a planktonic cell to a surface associated community involves physiological changes of individual cells and a spatiotemporal organization [60]. During surface attachment (Chap. 3.1.1), production of adhesions and extracellular matrix components is induced [61, 62]. After a successful attachment, microcolonies can be formed by clonal growth of the attached cells or aggregation with neighboring cells via bacterial surface motility (Chap. 3.1.2). Further bacterial growth and matrix production proceeds biofilm maturation. During this process, changes in biofilm size and architecture, extracellular matrix composition, and the physiological state of cells can be observed $[17,63]$. Finally, bacteria can detach from the mature biofilm and continue as planktonic cells until they attach again to a surface and repeat the biofilm development cycle [62]. 


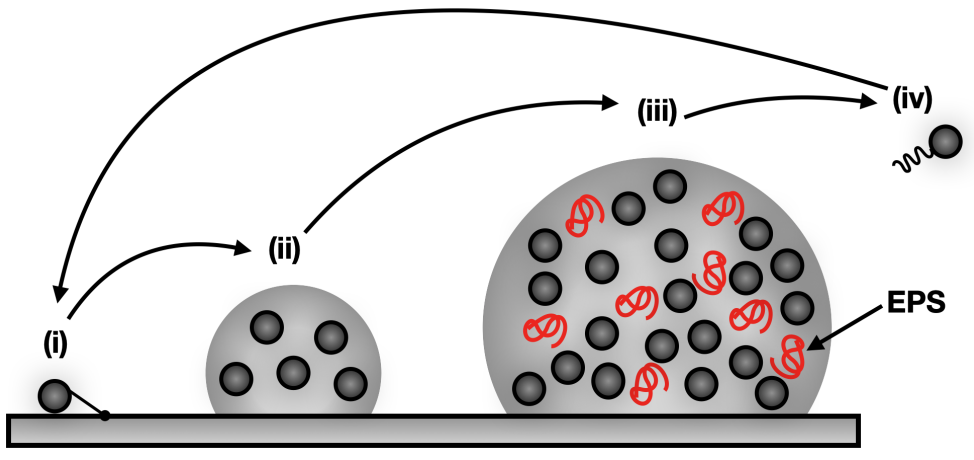

Figure 3.3: Stages of biofilm development. (i) Planktonic cells attach to a surface. (ii) Micro-colonies are formed due to cell growth or aggregation via cell motility. (iii) Biofilms matures due to further cell growth and expression of extracellular matrix components. (iv) Single cells can disperse from the biofilm and potentially start biofilm development at a new location.

\subsubsection{Structure and dynamics of biofilms}

A variety of physical parameters affect the structure of bacterial colonies and biofilms on a microscopical and mesoscopical scale. In order to study the structure and dynamics of biofilms, parameters like cell growth, cell-surface interactions and cell-cell interactions are important to access. Importantly, bacteria are able to adapt their biofilm structure to environmental stresses by tuning their physical interactions $[64,65,66,67,68]$. These interaction can be of repulsive or attractive nature (Fig 3.4). Attractive interactions can be mediated by extracellular appendages or molecules that rely on electrostatic attraction, polar attraction and hydrogen bonding (Fig 3.4a). Moreover, the presence of small, non-adhering extracellular matrix components inside biofilms can cause excluded volume effects. In order to minimize the excluded volume and maximize the entropy, bacteria tend to aggregate (Fig 3.4b). In contrast, bacteria are similar to hard objects that repel each other (Fig 3.4a). During cell growth, bacteria generate pressure that pushes their neighbors away. Additionally, a higher concentration of extracellular matrix components increases the osmotic pressure between the biofilm and its surrounding medium, causing biofilm swelling due to a higher medium influx (Fig 3.4d) [1]. 

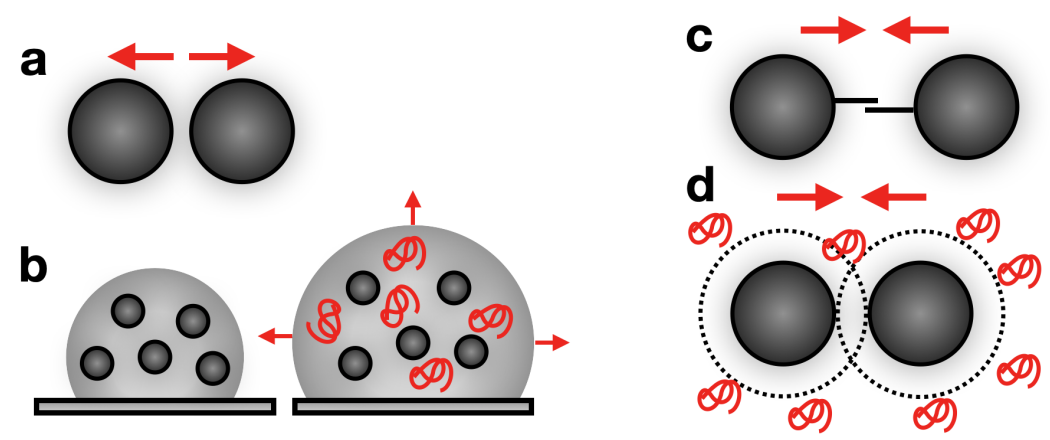

Figure 3.4: Repulsive and attractive interactions between bacteria. (a) Steric repulsion. (b) Osmotic pressure. (c) Bridging attraction. (d) Depletion attraction.

The cell shape influences the structure and growth dynamics of bacterial biofilms. Recently, bacterial biofilms formed by rod-shaped bacteria were intensely investigated $[69,63]$. At early stages of biofilm formation, E. coli and V. cholerae form a two-dimensional layer at surfaces that form patterns of nematic domains. The size of these nematic domains is influenced by the aspect ratio of cells and their growth rate (Fig. 3.5 a) [70, 69]. After a critical aggregate size, which depends on cell-surface interaction and cell length [71], the transition from two dimensional to three dimensional colonies occurs. To this end, the growth pressure has to overcome the cell-surface interaction [71].

For $V$. cholerae the growth of individual cells to a colony containing up to 10.000 cells was reconstructed using time resolved three dimensional images acquired by spinning disk confocal microscopy. Repulsive interactions in $V$. cholerae are mediated by osmotic pressure and steric repulsion (Fig. 3.4a,b), whereas attractive interactions are mediated by the adhesive protein RbmA that binds cells together $[56,72,73,74]$. The balance between repulsive and attractive interaction and their strength determine the colony structure, which shows local liquid-crystalline order [12]. Reducing cell-cell interaction though deletion of RbmA increases nematic order and space between cells [12]. Interestingly, growth rates were spatially homogeneous, even though cell growth depends on nutrient availability, which is reported to be spatially heterogeneous inside colonies $[12,7]$ (Sec. 3.2.3). The 
observed velocity of individual cells is determined by passive cell displacement during the fountain-like biofilm expansion (Fig. 3.5 b) and is spatially homogenous. For larger biofilms, the velocity increases slightly [12].

a

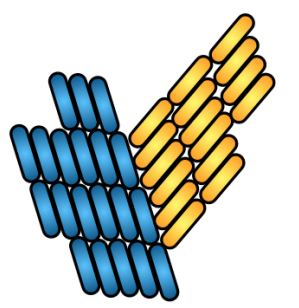

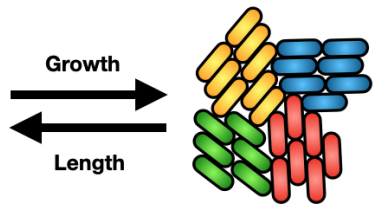

b

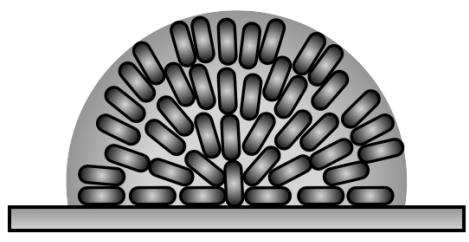

Figure 3.5: Biofilm structure of rod-shaped cells. (a) Domain size depends on growth rate and cell length. Higher growth rates decrease the domains size and a higher length increases them. (b) Fountain-like arrangement of rod-shaped cells.

In contrast to $V$. cholerae, the cell shape of $N$. gonorrhoeae is spherical. During growth, dumbbells are formed. In $N$. gonorrhoeae attractive interactions between cells are mediated by T4P [40]. Similar to twitching motility, cycles of pilus elongation, binding and retraction facilitates rapid three dimensional aggregation into spherical shaped colonies. To this end, pili of adjacent cells can bind to each other and pull the cell bodies closer together during pilus retraction. Due to the fact that the average force needed to rupture a pilus bond $F_{\text {rupture }} \approx 50 p N$ [44] is smaller than the force a single pilus can generate $F_{\text {pilus }} \approx 150 p N[45,33,46,47$, 48], it is likely that T4P-T4P bonds are transient (Fig. 3.6a). When many bacteria are in close proximity, a pilus network emerges that holds the cells together. The previously mentioned cycle of pilus elongation, binding, retraction and unbinding facilitates cell motility and cell reorganization in dense colonies as shown in silico [75]. 


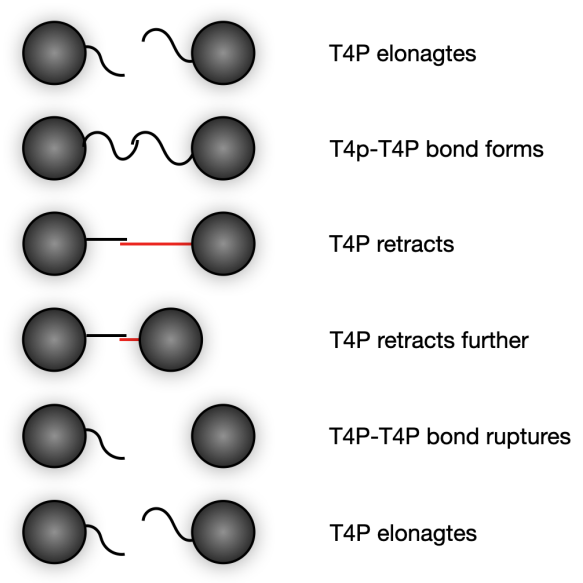

Figure 3.6: T4P-T4P interactions. Bridging attraction mediated by T4P. Cycles of $\mathrm{T} 4 \mathrm{P}$ elongation, binding, retraction and unbinding generate $\mathrm{T} 4 \mathrm{P}-\mathrm{T} 4 \mathrm{P}$ interaction patterns that facilitate motility and organization inside bacterial colonies.

Simulations computed repulsive interactions of dumbbell-shaped cells by volume exclusion and attractive interactions by T4P mediated forces. T4P were modeled as dynamics springs that can elongate, retract, bind and unbind. Similar to experiments, the simulated cells aggregate into spherical shaped colonies. The colony shape depends on the cooperation of cell-surface and cell-cell interactions. For stronger cell-surface interactions, the contact area to the surface increases. Stronger cell-surface interactions and a larger colony size increase the number of pili that interact with the surface and consequently, decrease surface-motility of the colony. Especially for small colonies, surface-motility enables colonies to move at surface, approach neighboring colonies, and coalesce with them into larger colonies $[76,44]$. Additionally, the simulations predicted a liquid-like behavior of gonococcal colonies by analysing local order and coalescence dynamics of two colonies. In order to maximize the number of interacting pili, outer cells aligned tangentially to the surface of colonies. However, cell orientations inside the colonies were randomly distributed and did not show nematic order. The computed RDF, which is used to measure order, showed local order similar to a liquid-like structure [75].

This simulation did not implement bacterial growth and lineage tracking of 
densely packed spherical cells that exceed the limit of optical resolution is complicated. Consequently, little is known about the spatio-temporal growth dynamics of colonies formed by spherical bacteria and how growth and physical interactions influence the structure of the colony.

\subsubsection{Phenotypic heterogeneity and stress response in biofilms}

Spatially heterogeneous regulation of cellular physiology maximizes the community fitness and allows better adaptation to global environmental changes [55, 77, 78, 79, 80, 81, 82]. During stress exposure, cells inside biofilms act collectively and adapt their biofilm structure [39, 83, 84]. In the following, molecular causes underlying phenotypical heterogeneity inside biofilms will be introduced and examples for different kinds of stress responses will be described.

\section{Nutrient starvation and stringent response}

Bacteria living in a dense community, experience time and position dependent local environments due to accumulation of waste products and emergence of gradients of nutrient accessibility (Fig. 3.7) [85, 7]. Cells located close to the periphery of biofilms, consume oxygen and nutrients, such that a smaller fractions can diffuse deeper into the aggregate. Especially, steep oxygen gradients were reported to cause hypoxic zones $[86,87,88,89,90,91]$, which reduces the metabolic activity and causes the cells to differentiate into a state comparable to the stationary phase [87, 91, 89, 90, 91]. The reduction in metabolic activity is assumed to contribute to higher antimicrobial tolerances, because antimicrobial agents are more efficient against fast growing cells compared to slow growing ones [92, 54, 93, 94, 95]. This assumption is additionally supported by an experiment that shows a higher antimicrobial tolerance of biofilms under global anaerobic conditions in comparison to the aerobic controls [89]. Additionally, bacteria in deeper layers of the biofilm are starving for carbon and nitrogen $[7,96,97]$ that also reduces metabolic activity $[98,99]$.

Different strategies evolved to enable or increase metabolic activity in the inner periphery. Bacillus subtilis biofilms are able to counteract against nutrient 
gradients by metabolic cooperation. They coordinate their metabolic activity to ensure a better nutrient supply for cells deep inside the biofilm, where nutrients are limited. Cells, which experience nutrient limitation, send spatially propagating waves of depolarization [100]. This electrical signal reduces the metabolic activity of cells in the outer periphery, such that more nutrients can diffuse into deeper layers $[101,100]$. Alternatively, cell autolysis is induced and provides nutrients and important matrix components [102].

If the costs of sustaining a biofilm outweighs its benefits, certain species act by initiation of a biofilm breakdown and cell dispersal [103]. Depletion of medium compounds like carbon, iron or oxygen were reported to be capable of inducing biofilm dispersal $[104,81,105,106,107]$. To transition efficiently from the surface associated biofilm to motile individuals, cells initiate matrix degrading to access additional nutrients and form dispersal units that should detach from the biofilm surface [103]. For example, Staphylococcus aureus actively triggers biofilm dispersion through production of matrix degradation enzymes, which are triggered by a collective cell level response to a global signal via quorum sensing $[108,109]$. $V$. cholerae responds both at the level of an individual cell and on a multicellular level via quorum sensing to make a robust decision regarding biofilm size and nutrient availability to start biofilm dispersal [110].

Another strategy to deal with nutrient limitation is the stringent response, which is triggered during amino acid, carbon or iron starvation [111, 112]. relA and spo $T$ are the key players in regulation of pentaphospate guanosine (pppGpp) and tetraphosphate guanosine (ppGpp). Both effector molecules can activate the stringent response [113] and are summarized to (p)ppGpp. The accumulation of (p)ppGpp supports the survival during starvation stress by downregulation of macromolecular synthesis (e.g. nucleic acid and protein synthesis) and upregulation of survival systems (e.g. systems involved in protein degradation) [111, 114]. Interestingly, independent studies show that stringent response contributes to the biofilm tolerance during antimicrobial treatment $[115,116,116]$. Nutrient supplementation increased biofilm susceptibility to antibiotics in comparison to the nutrient starving control $[117,116]$. Additionally, a reduced antimicrobial 
tolerance was obtained in $P$. aeruginosa biofilms formed by $\Delta$ rel $A \Delta s p o T$ mutants, where the stringent response was deactivated $[116,116]$.
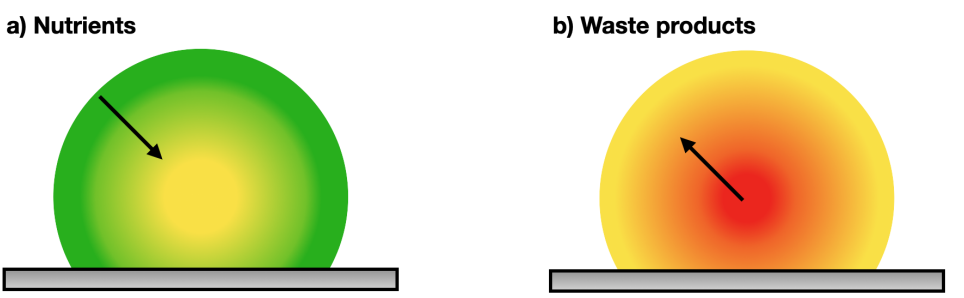

Figure 3.7: Chemical heterogeneity in biofilms. (a) Reaction-diffusion interactions leads to nutrient gradients (green). Concentrations of Oxygen or any nutrient that is consumed decreases with penetration depth into the biofilm. (b) Waste products are more concentrated inside the biofilm (red).

\section{Antimicrobial treatment}

Even though it has been known for decades that planktonic cells are more susceptible to antimicrobial treatment than genetically identical cells inside biofilms [2], the mechanisms behind the higher antimicrobial tolerance of biofilms have to be investigated further. Generally, several mechanism can cause an increased antibiotic tolerance that is reported to be the 1000-fold between P. aeruginosa biofilms and their planktonic counterpart [118].

The surrounding biofilm matrix was reported to be a diffusion limiting barrier that gives protection from penetration of bacteriophages [6] and some antibiotics like oxacillin, cefotaxime and vancomycin $[119,120,121]$. Interestingly, the production of curli amyloid fibers of E. coli biofilms is limited to cells located on the biofilm surface, but gives protection to the whole biofilm [6]. However, various antibiotics are able to penetrate different biofilm matrices without causing significant cell death $[8,122,123,124,125,126]$. The penetration of antibiotics through the biofilm is delayed due to the matrix. For example, the delay of tetracycline reaching all cells inside E. coli biofilms was measured to be $10 \mathrm{~min}$ [127]. It is assumed that the delay of antimicrobial penetration increases the time for a phenotypic response 
and consequently increases the antibiotic tolerance $[91,128]$.

Nonetheless, antimicrobial treatment of maturated biofilm can cause lethal damage. Currently, $V$. cholerae infections are typically treated with tetracycline [129], which inhibits protein translation and diffuses quickly into the biofilm. Prior emergence of significant cell death or a physiological response to translational inhibition, the biofilm architecture was modified during tetracycline exposure. On the single cell level, cells were still metabolically active and grew in size, but they could not divide due to missing divisome proteins [130]. On the multicellular level, attractive interaction that were mediated by an extracellular matrix compound (RbmA) were decreased during the treatment, causing a reduction in cell-packing density. As a result, treated biofilms showed a higher susceptibility to phages or invasion of other species. After $6 \mathrm{~h}$ of biofilm growth, cell death became significant [131].

The human pathogen $N$. gonorrhoeae causes gonorrhea and conjunctivitis. Due to a rapid development of antibiotic tolerances, the treatment of gonorrhea is hampered [15]. Currently, gonorrhea is treated with a combination of azithromycin and ceftriaxone [132]. Cronenberg et al. found that azithromycin treatment increases motility inside gonococcal colonies and the efficiency of a sequential ceftriaxone treatment. Simultaneous treatment of both antibiotics did not show any synergistic killing effect [5]. Even though the sequential treatment with azithromycin and ceftriaxone seems to be promising, the first resistant isolates with a combined resistance to azithromycin and ceftriaxone were reported in 2018 [133, 134]. Despite new promising antimicrobial treatments for gonorrhea like zoliflodacin are in sight [135], the number of possible antimicrobials is limited and the efficiency of future antimicrobials has to be optimized or alternative treatments have to be found. For example, Kim et al. found that commensal Neisseria strains have the ability to kill $N$. gonorrhoeae [136]. In particular, the killing effect is mediated when $N$. gonorrhoeae takes up the commensal external DNA and does not recognize its methylation pattern. It has been proposed that any DNA that has a different methylation pattern, homology to the pathogen genome, and can enter the pathogen, could be used as a potential microbicide [136]. 


\subsection{Cell and particle tracking methods}

Time resolved imaging is important to characterize bacterial growth dynamics and biofilm maturation. Due to progress in development of fluorescent proteins and optical microscopy technology, particle localization and tracking is possible inside bacterial biofilms. In order to analyse the acquired datasets, sophisticated automated particle tracking algorithms are needed. Particle tracking algorithms have been developed for the analysis of colloidal systems and can be adapted to localize single cells inside bacterial biofilms. In the following, different tracking approaches will be introduced, as well as their limitations.

In general, spinning disk confocal microcopy has proven to be useful for the analysis of short-time motility inside biofilms [50] and growth rates on longer time scales [12]. High temporal resolution can be achieved by acquisition of two dimensional image slices [50]. For three dimensional image acquisition the time resolution is limited to approximately $10 \mathrm{~min}$ [12]. In the near future light sheet microscopy will be a promising alternative to spinning disk confocal microscopy, allowing smaller time resolutions below 10 min due to its reduced phototoxicity and photobleaching technology [137]. Alternatively, microfluidic cultivation chambers are promising in cultivation and imaging two dimensional bacterial layers. Due to the restricted height, bacteria cannot grow into the third dimension. Bright-field imaging allows a high spatial and temporal resolution and analysis of motility and growth dynamics [138].

\subsubsection{Image segmentation}

Localization and tracking of particles in time-lapse confocal microscopy has been used to understand mechanical properties and dynamics of colloidal model systems of crystals [139, 140] or glass transition [141]. Later, particle tracking algorithms were adapted to track single cells inside biofilms to analyse cell motility and spatiotemporal growth dynamics $[12,50]$.

J. Crocker and D. Grier pioneered particle tracking by automatically finding features in two dimensional systems with sub-pixel accuracy using the programming 
language IDL [142]. Later their code was adapted for MATLAB. D. Blair and E. Dufresne simplified the original code for particle localization in two dimensions, where Y. Gao and M. Kilfoil optimized the code and implemented it also for three dimensional particle localization and tracking [143]. Particle tracking can be structured into four stages: image segmentation, particle localization, refinement of positions and linking time resolved positions of particles into trajectories.

The easiest way to segment colloids or cells from the background is to set a manually or automatically defined threshold that removes the background noise. This approach was applied successfully in many particle tracking algorithms [144, 145, 146, 147]. Prior to threshold definition, it can be necessary to increase the image signal to noise ratio (SNR) by image processing. To this end, J. Crocker and D. Grier used a real-space bandpass filter consisting of an boxcar average filter and a Gaussian-filter. The boxcar average filter models the background signal and the Gaussian-filter deals with the digitization noise. Both filters can be computed in a single convolution step by the kernel $K(i, j)=\frac{1}{K_{0}}\left[\frac{1}{B} \exp \left(-\frac{i^{2}+j^{2}}{4}\right)-\frac{1}{(2 w+1)^{2}}\right]$, where $K_{0}$ and $B$ are normalization factors and $w$ is the radius from the object of interest [142]. Alternatively, a watershed transform can be applied to segment the background noise from particle intensities [148, 149, 150, 151, 152, 153, 12]. The image is considered as a topographic relief, which is flooded from the local minima. As a result, the contours of objects (watersheds) are returned and the background noise is removed. When this algorithm is used, over-sensitivity to noise and over-segmentation can be a problem [149].

\subsubsection{Particle localization}

After image segmentation, the positions of colloids or cells have to be determined. Particle localization is typically achieved by applying a local maximum operator followed by a further refinement. J. Crocker and D. Grier calculated the offset $\epsilon$ between the locally brightest pixel $(x, y)$ and the brightness-weighted centroid $\left(x+\epsilon_{x}, y+\epsilon_{y}\right)$ with

$$
\left(\begin{array}{l}
\epsilon_{x} \\
\epsilon_{y}
\end{array}\right)=\frac{1}{m_{0}} \sum_{i^{2}+j^{2} \leq w^{2}}\left(\begin{array}{l}
i \\
j
\end{array}\right) A(x+i, y+j)
$$


where $m_{0}$ is the integrated brightness of the particle.

\subsubsection{Particle tracking}

The probability of finding single Brownian particle with a self-diffusion coefficient $D$ that diffused a distance $\delta$ between two frames is calculated by

$$
P(\delta \mid \tau)=\frac{1}{4 \pi D \tau} \exp \left(-\frac{\delta^{2}}{4 D \tau}\right) .
$$

Consequently, the probability distribution of $N$ identical non-interacting particles is

$$
P\left(\left\{\delta_{i}\right\} \mid \tau\right)=\left(\frac{1}{4 \pi D \tau}\right)^{N} \exp \left(-\sum_{i=1}^{N} \frac{\delta^{2}}{4 D \tau}\right) .
$$

In general, particle tracking algorithms link positions of successive frames into trajectories. They tend to find trajectory-sets that maximize the probability distribution $P\left(\left\{\delta_{i}\right\} \mid t\right)$ or the sum of displacements $\sum_{i=1}^{N} \delta_{i}^{2}$. Even though the tracking algorithm from J. Crocker and D. Grier assumes a non-interacting system, the algorithm is suggested to work well for weak-interacting spheres [142]. In order to limit the number of possible combinations of $P\left(\left\{\delta_{i}\right\} \mid t\right)$, only displacements that are shorter than a given distance $L$ are considered. As a result, the tracking network is divided into smaller sub-networks and the number of possible combinations reduces from $O(N$ !) to $O(M !)$, where $M$ is the number of linked particles inside a sub-network. The optimal cutoff distance for $L$ is in the range of $\delta<L<a / 2$, where $a$ is the distance between two neighboring particles. Y. Gao and M. Kilfoil use the base algorithm developed by J. Crocker and D. Grier [142], but they include a multipass strategy. In a homogeneous scenario, where particles have a small displacement compared to their neighbor distance, all particles can be tracked by one cutoff distance. In a dynamical heterogeneous scenario, where particles have different velocities, one cutoff is not sufficient anymore. Many tracks are lost, when the cutoff is too small. If the cutoff is too large, tracks get unreliable. To this end, Y. Gao and M. Kilfoil use their multipass strategy [143], which is described in chapter 5.4.4. 


\subsubsection{Alternative particle tracking tools}

The previously described localization algorithms are written for systems with identical spherical particles. They fail to access information about the size of individual particles. To address this shortcoming, Leocomach et al. developed a novel method to localize spherical particles of arbitrary sizes in either 2D or 3D images [154]. They found that very small particles are expelled from crystals and accumulated on the growth front of them [154]. This information was not accessible with standard particle tracking tools. In order to determine the position and orientation of rod-like colloids from confocal microscopy, Besseling et al. developed a new particle tracking algorithm, which should also work for ellipsoids and dumbbells with diameters down to $300 \mathrm{~nm}$. This algorithm is reported to be also applicable for active colloids like self-proppeled particles or bacteria [155].

In contrast to the previously mentioned tools that were primarily developed to analyse colloidal systems, the aim of COMSTAT is to characterize biofilm morphology. In particular, this tool can analyse quantitative parameters like biomass, roughness, surface area, volume and several more [156]. Even though this software was an important tool to quantify three dimensional biofilm structures [11], it cannot analyse internal properties of biofilms with cellular resolution.

Recently, the software tool BiofilmQ was published and allows the analysis of spatio-temporal dynamics inside biofilms with a focus on cell cytometry, data analysis and data visualization [157]. Hartmann et al., who developed BiofilmQ, successfully tracked single lineages of $V$. cholerae as they grew from a founder cell to 10.000 cells [12]. They tracked single rod-shaped cells by minimizing deviations in cell orientation and maximizing volume overlap. Assuming a constant cell width and a constant cell length between birth and division, growth rates were determined from cell tracking by measuring the cell length. 


\section{Aims of this study}

$99 \%$ of bacteria are estimated to live in surface associated biofilms and most infectious diseases are related to this sessile state $[158,159]$. Due to high tolerances of biofilms against disinfectants, cleaning and antimicrobial agents, new strategies have to be developed to treat unwanted and harmful biofilms that are responsible for industrial problems or chronic infections efficiently. To this end, different strategies have been developed, including prevention of biofilm formation [160], interfering or disrupting the biofilm structure to increase the sensitivity to the host defense system $[161,162,163,164]$ and killing biofilm cells through synergistic approaches, where the biofilm structure is dispersed and subsequently treated with conventional antibiotics [165]. In order to develop these strategies, information about the cellular structure and spatio-temporal dynamics of bacterial biofilms are required that are largely unknown for $N$. gonorrhoeae. Through attractive interactions mediated by their type 4 pilus system (T4P), gonococci rapidly organize in spherical microcolonies that grow with time, while maintaining their colony shape.

In the first part, we hypothesized that gonococcal colonies show liquid like properties that are influenced by T4P motor activity. Especially the spherical shape of gonococcal colonies and the fusion process of two adjacent colonies are reminiscent of liquid drops. The positions of single cells were determined and by calculating the radial and remoteness distribution function, the gonococcal colony structure was analysed. Additionally, we modified the motor activity of the gonococcal T4P machinery to study the effect on the colony structure.

In the second part, we hypothesized that cells inside gonococcal colonies show heterogeneous growth rates depending on their position. We aimed at characterizing the spatio-temporal growth dynamics inside gonococcal colonies.

Therefore, we combined two strategies with complementary advantages and 
disadvantages. The first strategy was to develop a lineage tracking method to estimate growth rates by counting the offspring of individual cells. The second strategy was to use the growth-induced flow field for determining growth rates. After successful implementation of these methods, we investigated to study spatiotemporal growth dynamics at different growth conditions. 


\section{Material and Methods}

\subsection{Cultivation and Growth Media}

In this study, the gram-negative bacterium Neisseria gonorrhoeae was used as a model organism. Cells were cultivated for approximately 15 hours at $5 \% \mathrm{CO}_{2}$ and $37^{\circ} \mathrm{C}$ on GC-agar plates (table 5.1). Using a plastic loop, cells were resuspended from GC-agar plates to liquid GC-medium (table 5.2). To generate bacterial stocks, cells were resuspended in Mili-Q water containing 10\% milk powder (Carl Roth, GC-Freeze) and rapidly frozen in liquid nitrogen. These stocks were stored at $-80^{\circ} \mathrm{C}$.

\begin{tabular}{|c|c|}
\hline $\mathrm{NaCl}_{\text {(Roth) }}$ & $5 \mathrm{~g} / \mathrm{l}$ \\
\hline $\mathrm{K}_{2} \mathrm{HPO}_{4}$ (Roth) & $4 \mathrm{~g} / \mathrm{l}$ \\
\hline $\mathrm{KH}_{2} \mathrm{PO}_{4}$ (Roth) & $1 \mathrm{~g} / \mathrm{l}$ \\
\hline Proteose Peptone No. 3 (BD) & $15 \mathrm{~g} / 1$ \\
\hline soluble starch (Sigma Aldrich) & $0.5 \mathrm{~g} / 1$ \\
\hline Bacto Agar (BD) & $10 \mathrm{~g} / 1$ \\
\hline Isovitalex & $10 \mathrm{ml} / 1$ \\
\hline
\end{tabular}

Table 5.1: GC-Agar was autoclaved and cooled to $55^{\circ} \mathrm{C}$ before $1 \%$ Isovitalex (table 5.3) was added. 


\begin{tabular}{|c|c|}
\hline $\mathrm{NaCl}$ (Roth) & $5 \mathrm{~g} / \mathrm{l}$ \\
\hline $\mathrm{K}_{2} \mathrm{HPO}_{4}$ (Roth) & $4 \mathrm{~g} / \mathrm{l}$ \\
\hline $\mathrm{KH}_{2} \mathrm{PO}_{4}$ (Roth) & $1 \mathrm{~g} / \mathrm{l}$ \\
\hline Proteose Peptone No. 3 (BD) & $15 \mathrm{~g} / \mathrm{l}$ \\
\hline Isovitalex & $10 \mathrm{ml} / \mathrm{l}$ \\
\hline
\end{tabular}

Table 5.2: GC-Medium was autoclaved and stored at $4{ }^{\circ} \mathrm{C}$. Prior usage, $1 \%$ Isovitalex (table 5.3) was added.

\begin{tabular}{|c|c|}
\hline $\mathrm{D}(+)$-Glucose (Roth) & $100 \mathrm{~g} / 1$ \\
\hline L-Glutamine (Roth) & $10 \mathrm{~g} / \mathrm{l}$ \\
\hline L-Cystein $\cdot \mathrm{HCl} \cdot \mathrm{H}_{2} \mathrm{O}$ (Roth) & $28.9 \mathrm{~g} / 1$ \\
\hline Cocarboxylase (Thiamine Pyrophosphate) (Sigma-Aldrich) & $0.1 \mathrm{~g} / 1$ \\
\hline Ferric Nitrate $\left(\mathrm{Fe}(\mathrm{NO} 3)_{3} \cdot 9 \mathrm{H}_{2} \mathrm{O}\right)($ Sigma-Aldrich $)$ & $0.02 \mathrm{~g} / \mathrm{l}$ \\
\hline Thiamine $\cdot \mathrm{HCl}$ (Roth) & $0.003 \mathrm{~g} / 1$ \\
\hline 4-Aminobenzoic Acid (PABA) (Sigma-Aldrich) & $0.013 \mathrm{~g} / 1$ \\
\hline$\beta$-Nicotinamide Adenine Dinucleotide (NAD) (Roth) & $0.25 \mathrm{~g} / 1$ \\
\hline Cyanocobalamin (Vitamin B12) (Sigma-Aldrich) & $0.01 \mathrm{~g} / 1$ \\
\hline
\end{tabular}

Table 5.3: Isovitalex aliquots were stored at $-20^{\circ} \mathrm{C}$

\subsection{Bacterial Strains}

All $N$. gonorrhoeae strains used in this study (Tab. 5.4) were supplied by co-workers and are based on wt ( $\mathrm{Ng} 150)$.

$\boldsymbol{w t}$ G4 motif was deleted by replacing it with the aac gene, which confers resistance against apramycin. The G4 motif is responsible for antigenic variation of the major pilin subunit pilE [166] and may generate heterogeneity by affecting pilus density and rupture force. To exclude the effect of antigenic varaition on colony dynamics and T4P-T4P interactions [167], the G4 motif was deleted.

wt green For the strain construction, the sfgfp gene was fused with the pilE promoter region $\left(P_{p i l E}\right)$. Subsequently, the fusion product PpilE-sfgfp was 
transformed into the $w t(\mathrm{Ng} 150)$ strain between the $\operatorname{lct} P$ and aspC loci.

$\boldsymbol{p i l T}_{\boldsymbol{W B 1}}$ The goal was to generate a strain with a reduced T4P retraction dynamics. To this end, pilT $T_{W B 1}$ produces a non-functional form of the retraction protein PilT. Due to the fact that PilT forms hexameric rings [168], we assumed to get hexameric rings with a mixture of functional and non-functional PilT, causing a negative effect on $\mathrm{T} 4 \mathrm{P}$ retraction dynamics. The activity of the ATPase PilT depends on the Walker A box for ATP binding and on the Walker B box for ATP hydrolysis. Non-functional pilT $T_{W B}$ was generated by replacing glutamate within the Walker $\mathrm{B}$ box by alanine. The relative level of functional and non-functional PilT depends on the promoter strength of the $p_{i l T_{W B}}$ genes. The pilT $T_{W B 1}$ strain expresses the $p_{i l T_{W B}}$ gene under the control of the IPTG-inducible lac promoter.

$\boldsymbol{p i l T}_{\boldsymbol{W B 2}}$ The $\operatorname{pilT}_{W B 2}$ strain expresses the $\operatorname{pilT}_{W B}$ gene under the control of the strong pilE promoter.

$\Delta$ pilT Without the retraction ATPase pilT, pilus polymers are still formed [169], but T4P loose their ability to retract and generate forces. Chromosomal DNA from strain GT17 was transformed into $w t$.

$\Delta \boldsymbol{r e l} \boldsymbol{A} \boldsymbol{\Delta} \boldsymbol{s p o T}$ The goal was to generate a strain that does not respond to nutrient starvation via stringent response, which is activated by the gene products of $\triangle \operatorname{rel} A$ and $\Delta s p o T$. This gene products adjust the level of the alarmone (p)ppGpp [113]. The production of (p)ppGpp is suppressed by the deletion of relA. To compensate the growth rate reduction that is cause by deletion solely relA, spoT is additionally deleted [170].

$\Delta$ relAsspoTgreen The fusion product PpilE-sfgfp was transformed into the $\Delta \operatorname{rel} A \Delta \operatorname{spoT}(\mathrm{Ng} 198)$ strain between the lctP and asp $C$ loci. 


\begin{tabular}{|c|c|c|}
\hline Strain & Relevant genotype & Source/Reference \\
\hline$w t(\mathrm{Ng} 150)$ & $G_{4}:: a a c$ & {$[171]$} \\
\hline wt green $(\mathrm{Ng} 194)$ & $\begin{array}{c}l c t p:: P_{\text {pile }} s f g f p s p e R:: a s p C \\
G 4:: a a c\end{array}$ & {$[172]$} \\
\hline $\operatorname{pilT}_{W B 1}(\mathrm{Ng} 171)$ & $\begin{array}{c}l c t p: P_{l a c P} p i l T W B e r m C: a s p C \\
G 4:: a a c\end{array}$ & {$[44]$} \\
\hline $\operatorname{pilT}_{W B 2}(\mathrm{Ng} 176)$ & $\begin{array}{c}\text { iga }:: P_{\text {pile }} \text { pilTW BermC } \\
\text { G4 }:: a a c\end{array}$ & {$[44]$} \\
\hline$\Delta p i l T(\mathrm{Ng} 178)$ & $\begin{array}{c}\text { pilT }:: m-T n 3 c m \\
\text { G4 :: aac }\end{array}$ & {$[173,44]$} \\
\hline$\Delta \operatorname{rel} A \Delta \operatorname{spoT}(\mathrm{Ng} 198)$ & $\begin{array}{c}\operatorname{rel} A:: \text { nptIIspoT }:: \text { erm } C \\
G 4:: a a c\end{array}$ & {$[172]$} \\
\hline$\Delta$ rel $A \Delta$ spoT green $(\mathrm{Ng} 224)$ & $\begin{array}{c}\text { rel } A:: \text { nptIIspoT }:: \text { erm C } \\
\text { lctp }:: P_{p i l E} s f g \text { fpspe } R:: \text { aspC } \\
G 4:: \text { aac }\end{array}$ & {$[172]$} \\
\hline
\end{tabular}

Table 5.4: List of used strains in this study 


\subsection{Choosing the experimental design}

A major challenge of this project was the detection and tracking of single bacterial cells within three-dimensional colonies. While tracking methods are well established for eukaryotic tissues whose cells are about ten-fold larger than bacterial cells, tracking of single bacteria remains difficult. Here, we developed experimental methods and software to overcome these difficulties by taking the advantages of CLSM and spinning disk confocal microscopy.

\subsubsection{Experiments to study the colony structure}

In order to study the structure of gonococcal colonies, we used a Leica TCS SP8 CLSM (CECAD Imaging Facility) with a 63x, 1.4 NA, oil immersion objective lens and an argon laser with an excitation wave length of $488 \mathrm{~nm}$. The software of the microscope allowed to increase the laser power as a function of the acquisition height. This was a critical feature, because cells below the focal plane absorb the laser light and and cause a decreasing intensity signal at larger heights. The optical resolution was maximized by decreasing the pinhole diameter of the microscope, leading to an automatically adjusted voxel size of $35 \mathrm{~nm}$ in $\mathrm{x}-\mathrm{y}$-direction and 130 $\mathrm{nm}$ in z-direction, and a total view of $59.65 \times 59.65 \mu \mathrm{m}$. Due to the long image acquisition time of about $20 \mathrm{~min}$, the sample needed to be fixated. For this experiment, photodamage was irrelevant and bleaching effects were negligible. As a result. high laser power were used that saturated the image intensity signal. Samples were prepared as follows.

Cells were grown on GC + IsoVitaleX plates over-night at $37^{\circ} \mathrm{C}, 5 \% \mathrm{CO}_{2}$ and re-suspended in liquid GC-medium, to an optical density of 0.01 at a wavelength of $600 \mathrm{~nm} .300 \mathrm{\mu l}$ of cell suspension was inoculated in our flow chamber setup, which consisted of an ibidi $\mu$-Slide $\mathrm{I}^{0.8}$ Luer flow chamber with a glass bottom. The outlet of flow chambers was connected via Elbow luer connector (Ibidi) and silicon tubing to a waste flask and a peristaltic pump (model 205U; Watson Marlow, Falmouth, United Kingdom) for constant nutrient supply. The inlet of flow chamber was connected to a growth-medium reservoir via a self-build bubble trap. To build a bubble trap, the lower part of an $1 \mathrm{ml}$ syringe was closed with a $500 \mathrm{\mu l}$ reaction 
tube lid and a hole was drilled to connect the silicon tubing. This bubble trap contained a reservoir directly above the inlet of the flow chamber. Instead of entering the channel of the flow chamber, bubbles moved to the top of the bubble trap.

The flow chamber setup was filled with GC-medium and $200 \mu \mathrm{l}$ of cell suspension were injected into the flow chamber using a syringe. Prior to switching the flow on, cells were left for $1 \mathrm{~h}$ at $37^{\circ} \mathrm{C}$ for attachment to the glass surface. After 6 or $24 \mathrm{~h}$ of growth, biofilms were stained with $10 \mu \mathrm{M}$ Syto9 (Thermo Fisher Scientific) for $15 \mathrm{~min}$ at $37^{\circ} \mathrm{C}$. Subsequently, the biofilms were fixed with $4 \%$ paraformaldehyde in $1 \mathrm{x}$ PBS for 15 min at room temperature and washed with $1 \mathrm{x}$ PBS. Staining, fixation and mounting procedures were performed as following: the peristaltic pump was turned off, a silicon tubing between growth reservoir and flow chamber was pinched off, the lid of the bubble trap opened and the preferred solution filled into the bubble trap. Finally, the peristaltic pump was turned on again, such that the solution was injected automatically into the flow chamber in a well controlled manner. In the end, the bubble trap was closed and the clamp removed.

To prepare $30 \mathrm{ml}$ of $4 \%$ paraformaldehyde, $1.2 \mathrm{~g}$ paraformaldehyd was added to $19 \mathrm{ml}$ of $\mathrm{MQ}$ water and heated to $60^{\circ} \mathrm{C}$. In a fume hood, $10 \mu \mathrm{l}$ of $5 \mathrm{M} \mathrm{NaOH}$ was added. Adding $\mathrm{NaOH}$ to the solution was continued until the paraformaldehyd was dissolved. $10 \mathrm{ml}$ of $3 \mathrm{x}$ PBS was added to the solution and the $\mathrm{pH}$ was set to 7.2 by adding small aliquots of $5 \mathrm{M} \mathrm{HCl}$. The solution was filled with MQ water to the final volume of $30 \mathrm{ml}$. Finally, the solution was filtered through a 0.45 micron filter and stored at $-20^{\circ} \mathrm{C}$. To reduce fluctuations during image acquisition the samples were mounted with Mowiol (Table 5.5).

\subsubsection{Experiments to study the spatio-temporal growth dynamics}

In contrast to the experiments that were performed to study colony structure, the samples used to study growth dynamics needed to be alive. To increase acquisition speed and reduce photodamage of individual cells an inverted microscope (Ti-E, Nikon, Minato, Japan) equipped with a spinning disc confocal unit (CSU-X1, 


\begin{tabular}{|c|c|}
\hline Mowiol 4-88 & $2.4 \mathrm{~g}$ \\
\hline Glycerol & $6 \mathrm{~g}$ \\
\hline MQ water & $6 \mathrm{~mL}$ \\
\hline 0.2 M Tris-Cl $(\mathrm{pH} 8.5)$ & $12 \mathrm{~mL}$ \\
\hline DABCO & $2.5 \%$ \\
\hline
\end{tabular}

Table 5.5: Mowiol 4-88 was mixed with Glycerol and MQ water. The solution was mixed using a magnetic stir bar for several hours at room temperature. After adding Tris- $\mathrm{Cl}(\mathrm{pH} 8.5)$ the solution was heated to $50{ }^{\circ} \mathrm{C}$ for $10 \mathrm{~min}$ with occasional mixing. When Mowiol was dissolved, it was centrifuged at $5000 \mathrm{~g}$ for $15 \mathrm{~min}$. The supernatant was carefully removed and DABCO was added to $2.5 \%$ to reduce bleaching. Stocks were stored in $-20^{\circ} \mathrm{C}$ freezer.

Yokogawa, Tokyo, Japan) and a 100x CFI Apo Tirf objective (Nikon, Minato, Japan) was used. This spinning disk confocal microscope reduced the acquisition time to several seconds. By minimizing the laser power and exposure time a time resolution of 15 min between image stacks was achieved. The software of the microscope recommended a voxel size of $80 \mathrm{~nm}$ in $\mathrm{x}$-y-direction and $200 \mathrm{~nm}$ in z-direction. To reduce the photodamage by the laser further, we increased the voxel size in z-direction to $400 \mathrm{~nm}$. For each time-point, three channels were acquired. The signal of sfgfp expressing cells was captured in green channel with the excitation laser wavelength of $488 \mathrm{~nm}$. Dead cells were stained by PI and were fluorescent in the red channel with the excitation laser wavelength of 561 nm. Information about positions and sizes of colonies could not be extracted from image stacks taken in the green channel, because we mixed green fluorescent and non-fluorescent cells. To this end, we captured the states of whole colonies with bright-field images of the home-position (bottom of flow chambers). The preparation of samples deviates from experiments that were performed to study colony structure. Experiments to study growth and death within bacterial colonies were performed as follows.

Fluorescent ( $s f g f p$ ) and non-fluorescent cells were grown on GC + IsoVitaleX plates over-night at $37^{\circ} \mathrm{C}, 5 \% \mathrm{CO}_{2}$ and re-suspended to liquid GC-medium. In 5 ml GC-medium, the optical density of fluorescent and non-fluorescent cells was set to 0.1 with a ratio of 1 to 100 . To ensure a proper cell mixture, the solution 
was supplemented with $100 \mu \mathrm{l}$ pure water and incubated for $30 \mathrm{~min}$ in the shakingincubator at $37^{\circ} \mathrm{C}, 5 \% \mathrm{CO}_{2}$. Supplementation of small quantities of water induced colony disassembly for an unknown reason. The long incubation time allowed assembly of new aggregates. Subsequently, $500 \mu \mathrm{l}$ of this solution was diluted with $500 \mu \mathrm{l}$ GC-medium, prior to injecting $250 \mu \mathrm{l}$ into the flow chamber-setup.

To enable time resolved image acquisition, it was necessary to disable or reduce the motility of colonies that can be observed on glass-surfaces. For this purpose, we used tissue culture treated ibidi $\mu$-Slides $\mathrm{I}^{0.8}$ Luer flow chambers with a polymer coverslip. In order to coat the flow chambers, they were filled with $200 \mu$ Poly-Llysine (Sigma, stock solution $50 \mathrm{\mu g} / \mathrm{ml}$ ) at least one day before the experiment. After one hour Poly-L-lysine was rinsed out of the flow chamber and dried for several hours at room temperature.

The flow chamber setup was assembled by connecting the outlet via Elbow luer connector (Ibidi) and silicon tubing to a waste flask. Then, the inlet was connected via a self-build bubble trap to a $90 \mathrm{ml}$ growth-medium reservoir, containing $4 \mathrm{\mu l}$ propidium iodide (PI). To build the bubble trap, the lower part of a $2 \mathrm{ml}$ syringe was sealed with the lid of a $2 \mathrm{ml}$ reaction tube and a hole was drilled to connect the silicon tubing. This bubble trap contained a small reservoir directly above the inlet of flow chambers. Instead of entering the channel of flow chambers, bubbles moved to the top of the bubble trap. Fresh-GC-medium was pumped constantly at $1 \mathrm{rpm}$ by a peristaltic pump (model 205U; Watson Marlow, Falmouth, United Kingdom).

\subsection{General tracking procedures}

\subsubsection{Procedures for image segmentation}

The CLSM and the spinning disk confocal microscope generated very different data sets in respect to image composition, SNR, resolution and size. Before particle localization algorithms can be applied, all image stacks have to be segmented to provide a reliable particle localization. 


\section{Segmentation of images acquired by CLSM}

Images acquired by a CLSM showed a low background signal. All image stacks were filtered using a three dimensional spatial bandpass filter to reduce the pixel noise and the background signal, while retaining the information of the characteristic feature size. We used the bandpass filter that was written by Y. Gao and M. Kilfoi. The algorithm is based on the IDL code from J. Crocker and D. Grier (Chap. 3.3.1) [142]. In order to reduce computation time, the convolutions were computed on the GPU.

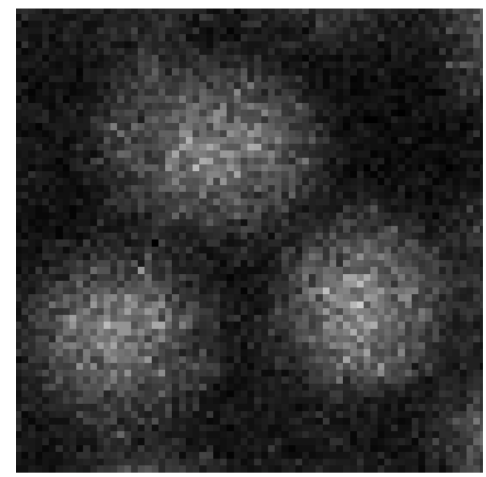

(a)

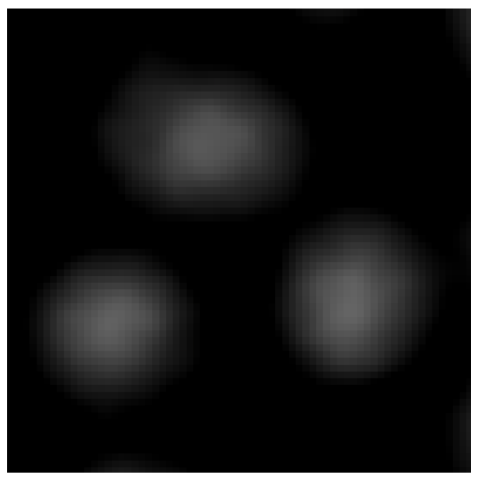

(b)

Figure 5.1: Effect of a spatial bandpass filter applied on image stacks acquired with a CLSM. (a) Section from original image. (b) Same image section after bandpass filter application.

\subsubsection{Segmentation of images acquired by spinning disk confocal microscopy}

Images acquired by a spinning disk confocal microscope showed a reduced image SNR and resolution compared to images acquired by a CLSM. Consequently, the spatial bandpass filter was not sufficient anymore to provide a reliable particle localization. We developed a novel image segmentation algorithm that reduces the background noise effectively (Fig. 5.2). Additionally, the algorithm enables to distinguish between the peaks of dumbbell-shaped cells. This is a crucial property that enables a reliable particle localization and to count the offspring of individual 
cells.

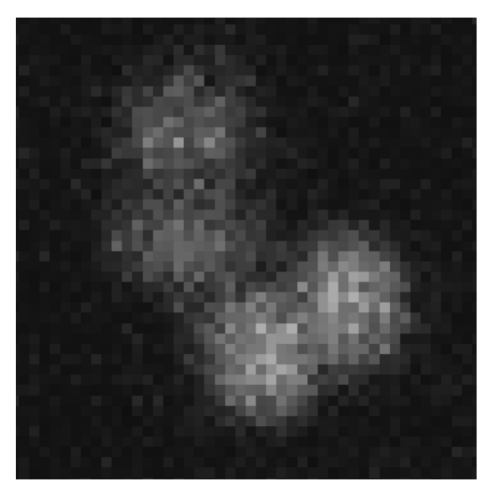

(a)

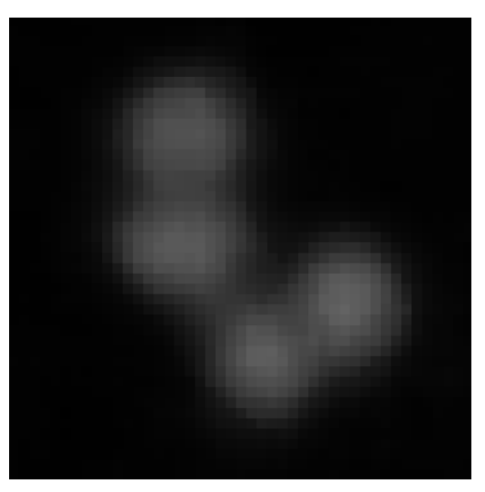

(b)

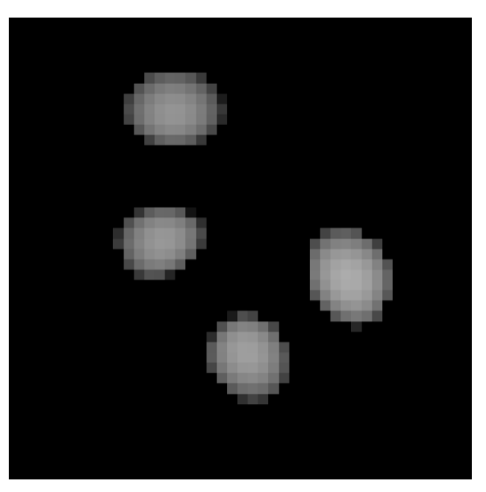

(c)

Figure 5.2: Image segmentation of image stacks acquired with a spinning disk confocal microscope. (a) Section from original image. (b) Same image section after applying median-filter. (c) Image section after all filters were applied.

At first, a median-filter was applied to get rid of single very bright voxels (Fig. 5.2b). Due to a different pixel resolution in z-direction compared to $\mathrm{x}-\mathrm{y}$ direction, the particles appear to be squeezed together in z-direction. To solve this issue, the 3D-images were resized by repeating every image slice three times in z-direction to make the particle intensity profiles spherical. To reduce the background noise, the mean filtered image was subtracted from the original image. Subsequently, the image contrast or the signal to noise ratio was increased by taking each intensity value to the power of 3. 59 intensity profiles of spherical particles have been manually saved and a parabola $f(x)=a x^{2}+b x+c$ was fitted to the data (Fig. 5.3a). Then a 3 dimensional kernel (Fig. 5.3b) was defined with $f(x)=a(x-b)^{2}+a(y-b)^{2}+a(z-b)^{2}+c$. The negative part of the kernel (Fig. $5.3 \mathrm{~b})$ is important to ensure lower intensity values for voxels which are located between dumbbell peaks (Fig. 5.3c) and to reduce noise. Without the negative part of the kernel, two peaks in close proximity with a noisy intensity profile could not been distinguished from each other. But the partially negative kernel showed an artifact. The distance between two peaks increased due to the negative part (Fig . 5.3d) and was consequently slightly overestimated. However, we were mainly 


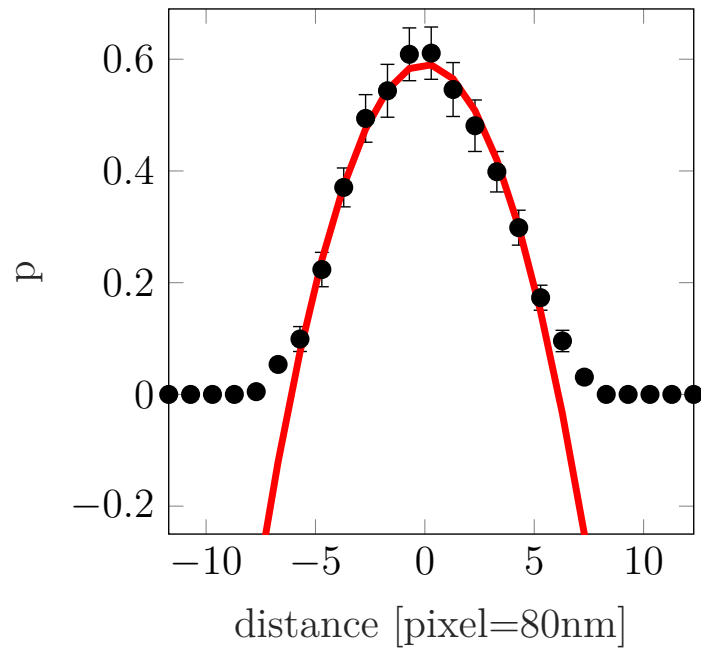

(a)

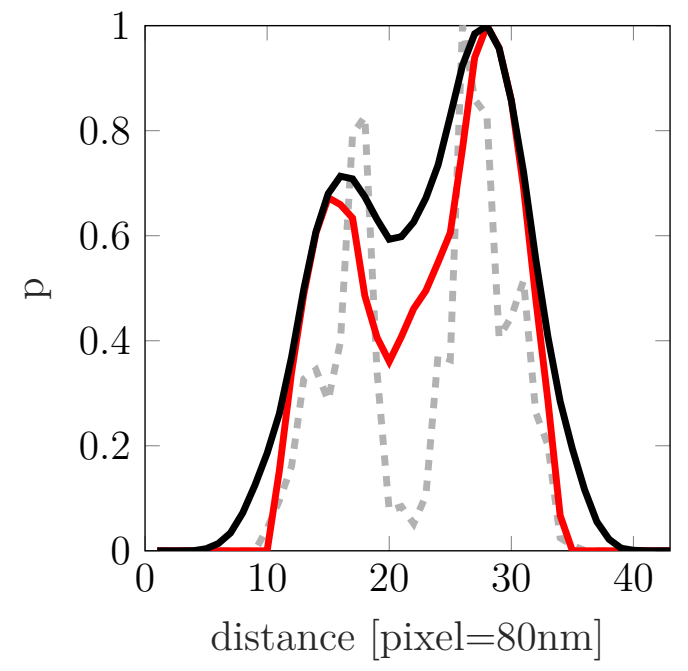

(c)

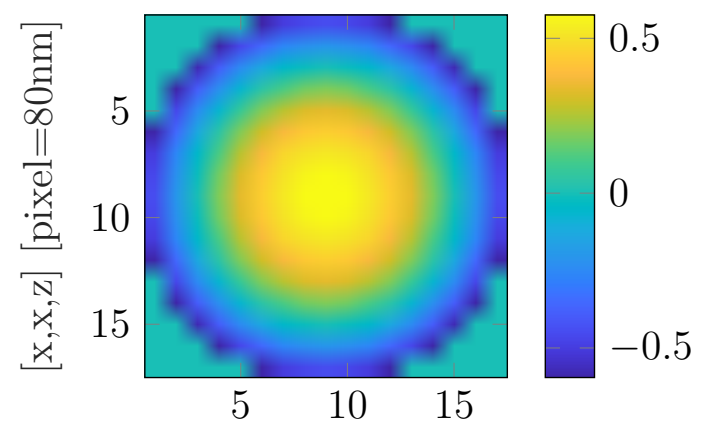

$[\mathrm{y}, \mathrm{z}, \mathrm{y}][\mathrm{pixel}=80 \mathrm{~nm}]$

(b)

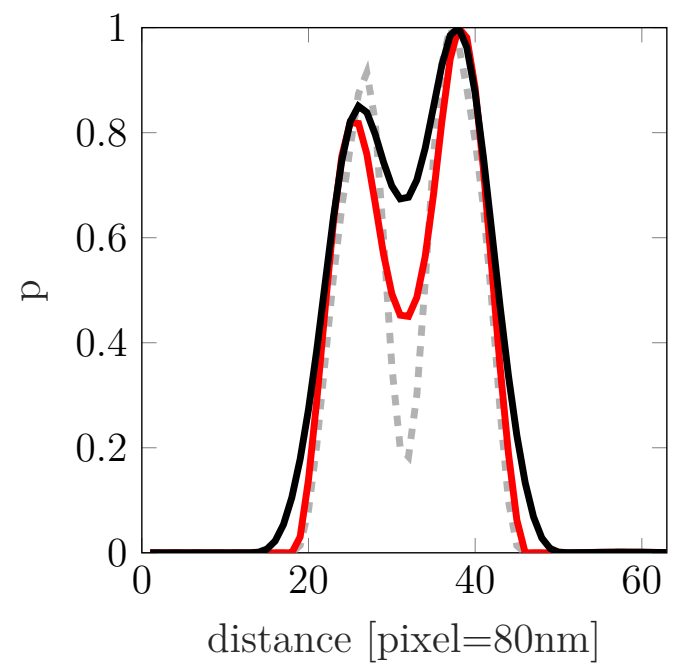

(d)

Figure 5.3: (a) Intensity profile of spherical particles. Black: mean values with standard error. Red: Parabola fit $f(x)=a x^{2}+b x+c$. (b) 3d-matrix is generated with the parabola fit using $f(x)=a(x-b)^{2}+a(y-b)^{2}+$ $a(z-b)^{2}+c$. The convolution of $3 \mathrm{~d}$-images with this matrix shows a strong reduction in noise and increasing intensity values for particles. Due to the negative part of the kernel, it is possible to distinguish between close intensity peaks. (c) 1d-convolution of a dumbbell shaped intensity profile with the parabola fit. Black: Parabola without the negative part. Red: Parabola with the negative part. (d) Dumbbell shaped intensity profile is blurred before convolution. 
interested in the total number of cells, such that this effect was irrelevant. So we applied a 3D-convolution of our image stacks with this kernel (Fig. 5.2c).

\subsubsection{Procedures for localizing individual cells}

In order to localize particles, the objects of interest have to be distinguished from the background noise. To this end, image segmentation has to be adapted to the acquired image stack and is often a necessary step prior particle localization. After determining the positions of cells with pixel-accuracy via a local maximum operator, Y. Gao and M. Kilfoil used the fractional-shifting method to improve the localization accuracy for three dimensional features [143]. The fracshift-kernel is calculated for the first iteration step by:

$$
\begin{aligned}
& \left(\begin{array}{c}
\epsilon_{x} \\
\epsilon_{y} \\
\epsilon_{z}
\end{array}\right)=\frac{1}{M} \sum_{i^{2}+j^{2}+k^{2} \leq w^{2}}\left(\begin{array}{l}
i \\
j \\
k
\end{array}\right) I(m+i, n+j, p+k) \\
& I=I_{1} *\left(1-\left|\epsilon_{x}\right|\right) *\left(1-\left|\epsilon_{y}\right|\right)+I_{2} *\left|\epsilon_{x}\right| *\left(1-\left|\epsilon_{y}\right|\right) \\
& +I_{3} *\left(1-\left|\epsilon_{x}\right|\right) *\left|\epsilon_{y}\right|+I_{4} *\left|\epsilon_{x}\right| *\left|\epsilon_{y}\right| \\
& I=\left\{\begin{array}{c}
I_{q} *\left|\epsilon_{z}\right|+I_{q+1} *\left(1-\left|\epsilon_{z}\right|\right), q=p \text { if } \epsilon_{z} \geq 0 \\
I_{q} *\left(1-\left|\epsilon_{z}\right|\right)+I_{q+1} *\left|\epsilon_{z}\right|, q=p-1 \text { if } \epsilon_{z}<0
\end{array}\right.
\end{aligned}
$$

where $M$ is the integrated intensity and $w$ is the radius of the mask. For the first step, the pixels of the kernel are in registry with those in the image. When the centre is updated to $\left(m+\epsilon_{x}, n+\epsilon_{y}, p+\epsilon_{z}\right)$, the intensity $I$ is calculated by a linear interpolation of the involved voxels in the image, based on their volume contribution. In three dimensions, the intensity contribution of 8 voxels are involved, when a voxel is shifted to $\left(m+\epsilon_{x}, n+\epsilon_{y}, p+\epsilon_{z}\right)$. These 8 voxels are composed of 4 voxels from the image plane $p$ and 4 voxels from the upper image plane $p+1$. To reduce pixel biasing for three dimensional particle localization, 20 iteration steps are needed and in two dimensions one iteration step is sufficient [143]. 


\subsubsection{Procedures for tracking cells}

The localized objects have to be linked from frame to frame. In order to track all particles with $100 \%$ fidelity, Y. Gao and M. Kilfoil developed a multipass strategy that is superior to other particle tracking program in handling heterogeneous dynamics [143]. They start with a small cutoff to get trajectories with the smallest displacement and subsequently delete the corresponding positions. The cutoff value is increased and the procedure repeated until the maximal displacement is reached. The goal is to obtain as many long trajectories as possible. Hence, the parameter good, which defines the minimal length of trajectories, is also variable. In the first step, good is set to the longest trajectory, which is the number of frames acquired. Starting from the smallest cutoff, all cutoffs are tested and successful tracked positions deleted. Then, good is set to the second longest trajectory and so on until the minimal trajectory length is reached. Thus, the longest trajectories with the smallest displacement are tracked first and shorter trajectories with larger displacements later. 


\subsection{Effects of parameter variation on the RDF}

The particle localization algorithm from Y. Gao and M. Kilfoi [143] was used to determine three dimensional positions of cells after image segmentation. The bacterial diameter and the minimal separation distance between two cells has to be set manually. Variation of these parameters was analyzed with the RDF for all positions determined from the CLSM.

The RDF is typically used to analyse hard-sphere fluids at different densities. It computes the probability of finding two particles with a distance $r$ apart, normalized with the probability of finding the same distance in an ideal gas at the same density (Eq. 5.5), [174]. All distances $n(b)$ between pairs of particles are determined and sorted into bins $b$ with the width $d r$ in the intervals $[r, r+d r]$. The density $\rho$ has to be determined from the distribution of particles to compute the distribution of distances $n_{i d}(b)$ in an ideal gas (eq. 5.4). To estimate the particle density, the distribution of distances in an ideal $n_{i d}(b)$ (eq. 5.4) was fitted to the distribution of distances between two particles $n(b)$. Thus, we guaranteed that the RDF converges to 1 for larger distances without explicitly determining the density $\rho$.

$$
\begin{gathered}
n_{i d}(b)=\frac{4 \pi \rho}{3}\left[(r+d r)^{3}-r^{3}\right] \\
g\left(r+\frac{1}{2} d r\right)=n(b) / n_{i d}(b)
\end{gathered}
$$

We noticed that variation of the main input parameters for the particle localization algorithm influence the RDF. Variation of the Gaussian-width of the bandpass-filter kernel did not influence the RDF considerably (Fig 5.4a). When we increased the bacterial diameter parameter, we observed slightly lower RDF values for small distances $r$ (Fig 5.4b). We set the bacterial diameter to $0.7 \mu \mathrm{m}$, because this distance was in good agreement with electron microscopy images [31]. Reducing the minimal separation distance showed the largest effect on the RDF (Fig 5.4c), causing a larger amount of small distances $r$ around $0.5 \mu \mathrm{m}$. Overlap of particle intensity profiles in z-direction due to the point spread function of the microscope could cause an unwanted peak between these particles, which could 
have been localized by the a smaller minimal separation distance. However, it is more likely that the lower minimal separation distance registered two separate peaks of growing cells that showed a dumbbell shape prior cell division. We decided to use a minimal separation distance of $70 \%$ of the cell diameter $(0.49 \mu \mathrm{m})$, because the main RDF peak was the highest at this value.
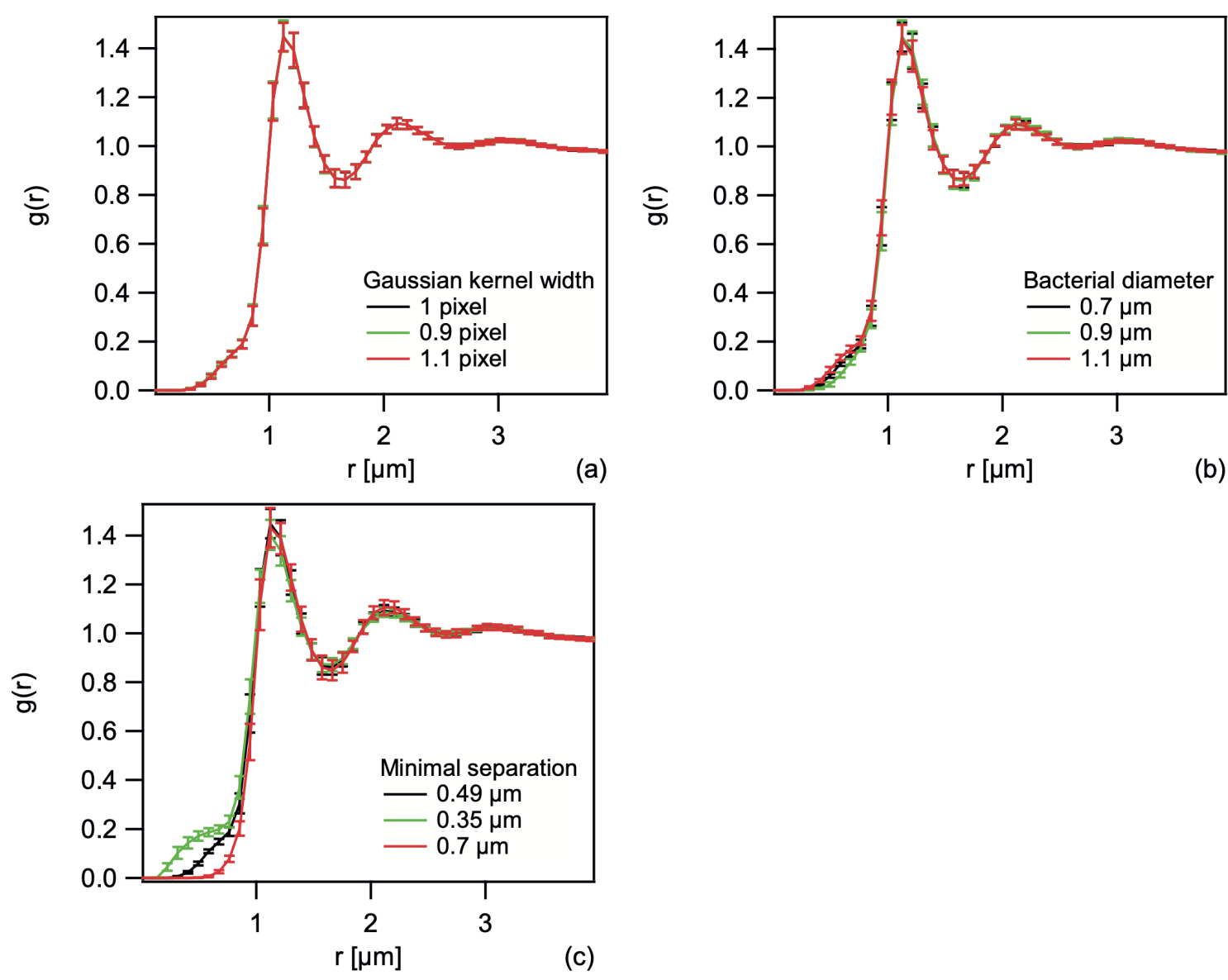

Figure 5.4: Effect of parameter variation for coordinate determination on radial distribution function $g(r)$. wt $(\mathrm{Ng} 150)$ colonies were analyzed after 6 h; error bars: standard deviation of 15 colonies from 3 independent experiments. $g(r)$ for (a) varying width of the Gaussian filter, (b) bacterial diameter, and (c) separation between the bacteria and the mask around the bacterial positions. Image adapted from [44]. 


\subsection{Implementation of particle tracking}

Prior applying the particle tracking algorithm from Y. Gao and M. Kilfoi [143], positions of cells needed to be corrected in respect to the centre of mass (COM) of colonies. To this end, bright-field images (Fig. 5.5a) were analyzed to get information about positions of colonies. In order to determine the colony contour, the goal was to process the images in a way that a threshold can be used to distinguish between the signal of the colony and the background noise.

Image contrast was increased by taking every pixel to the power of 2 and a median filter was applied. The signal distribution inside the colony showed peaks and troughs with a characteristic width $d$. Two different filters were applied, which replaced the intensity of every pixel by the maximal (I1) or minimal (I2) value of their $d / 2$ proximity. By inverting I2 and multiplication with I1, the resulting image (I3) showed a smoothed intensity distribution a high contrast to the background noise. A gaussian-filter was applied on the image I3. Through a threshold, the colony area was determined (Fig. 5.5c). A circle was fitted to the colony contour (Fig. 5.5d). Subsequently, the goodness of the fit was determined by the mean deviation of colony contour and the fitted circle. Estimates from bad fits were discarded. Additionally, estimates at time $t$ that deviated to much from the estimates determined at time $t-1$ and $t+1$ were also discarded. In order to fill the resulting data gaps at time $t$, they were replaced by the mean value from $t-1$ and $t+1$. Finally, the estimated radii were corrected by a factor of 1.2 .

The particle tracking algorithm fails for high cell densities. In order to reduce the density of fluorescent cells further, single cells with a maximal distance of 2 $\mu \mathrm{m}$ from each other apart were assigned into clusters. The mean position of all cells inside clusters at a certain time $t$ represents the coordinates of clusters. The particle tracking algorithm tracked the positions of clusters and generated a matrix containing the three dimensional cluster coordinates, the corresponding number of features, time, distance to the origin and cluster identification numbers. 


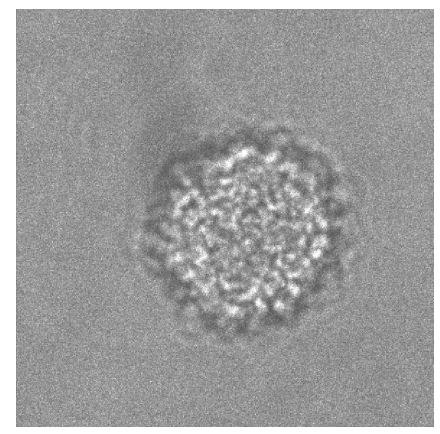

(a)
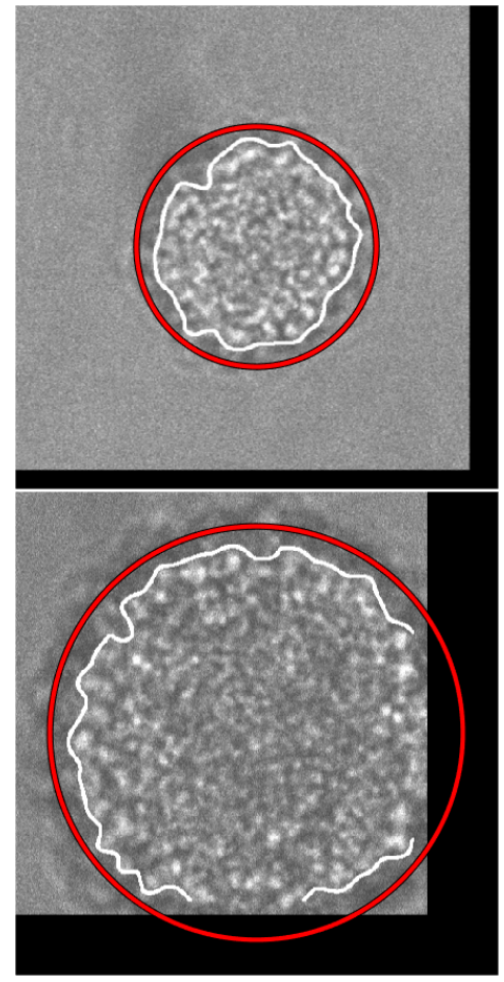

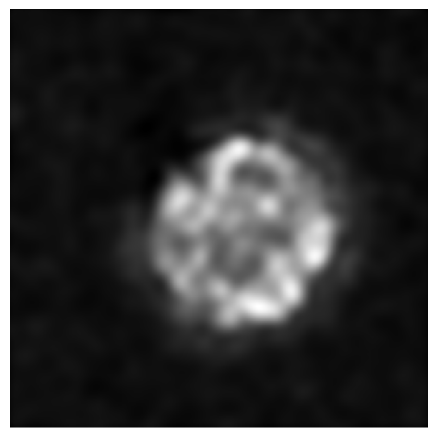

(b)

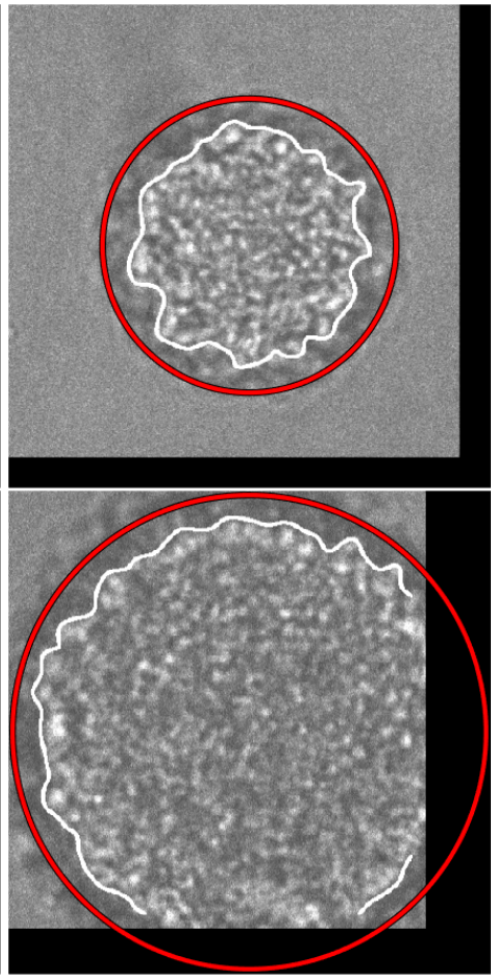

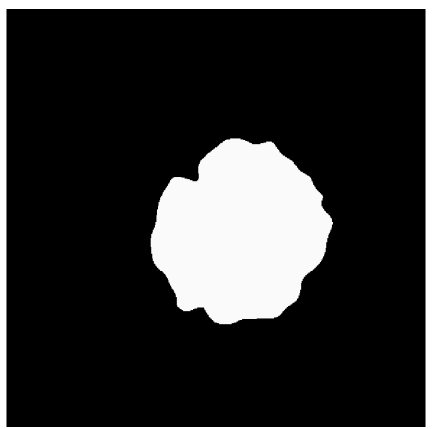

(c)

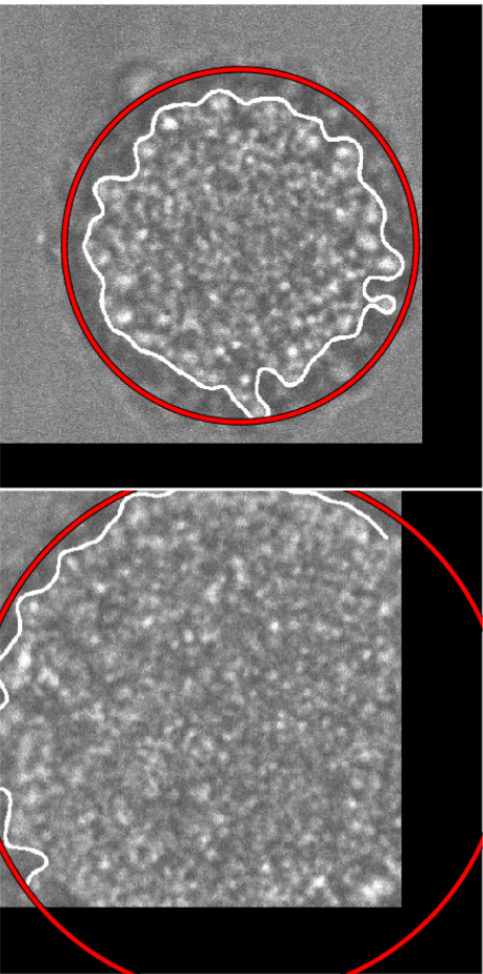

(d)

Figure 5.5: (a) Bright-field image of an early wt colony. (b) Self-written filter was applied. (c) Setting a threshold gives the corresponding binary image. (d) Time-series of an $w t$ colony with $d t=1.25 h$. Bright-field images were registered, such that the COM of colonies was located in the image centre. White lines: Contour of binary image. Red circles: Estimated colony edge by fitting a circle to the colony-contour and multiplying this value by a factor of 1.2 to get the real radius $r$.

From the cluster trajectories, we were able to optimize the estimation of the 
height $h_{\text {colony }}$ of the COM of the colony. We observed that $h_{\text {colony }}$ depends on the colony radius. The larger the colony radius was, the higher was the COM of the colony. Hence, we tested different ratios of radii of colonies to estimate $h_{\text {colony }}$. We searched manually for a ratio, where the displacements of clusters were symmetrical to the origin to estimate $h_{\text {colony }}=0.85 * r_{\text {colony }}$.

\subsection{Limitations in particle localization and tracking}

\subsubsection{Pixel-biasing and SNR influence particle localization}

Optical limitations of typical confocal microscopes reduce the maximal image resolution to $200-300 \mathrm{~nm}$ in the lateral and 500- $700 \mathrm{~nm}$ in the z-direction [175, 176]. Due to the point-spread-function of the microscope, which is caused mainly by diffraction from the objective [177], the cell body elongates in z-direction. When cells are aligned along the z-axis, their intensity signal can overlap and lead to an overestimation of particle size and errors in localization [154]. Given that the bacterial cell body is approximately $1 \mu \mathrm{m}$ in diameter, its size exceeds the resolution of confocal microscopy only 2-3 fold. Consequently, pixel-biasing, which influences the accuracy of cell-localization, confers a problem. In general, pixel-biasing describes the phenomenon, where the localized sub-pixel position of a particle is biased towards the centre of a pixel/ voxel. Using the fracshift method from Y. Gao and M. Kilfoil, one iteration step is sufficient to suppress pixel-biasing in two dimensions, but for three dimensional particle localization 20 iteration steps are needed, because the $\mathrm{x}-\mathrm{y}$-data suffers from the worse z-resolution [143].

Moreover, the SNR was reported to be the critical factor for localization algorithms $[178,179]$. The SNR strongly depends on the particle density, acquisition height, laser power and on the used fluorophor. A high laser power and exposure times increases the image SNR, but potentially causes lethal photodamage and photobleaching of single bacterial cells. Free radicals emerge from bleached fluorophores due to illumination, which are toxic to cells [180]. Consequently, a balance has to be found between an acceptable photodamage of the specimen, a sufficient image quality and spatio-temporal resolution. The choice of these coupled parameters and the experimental design strongly depends on the goal and 
the bacterial species of interest.

Even though high laser powers were used during image acquisition of fixated samples with a CLSM, the SNR was the limiting factor for particle localization (Fig 5.6a). Close to the surface, the background noise was very small and low laser powers could be used to distinguish between fluorescent cells and background noise (Fig 5.6b). As a function of penetration depth into the fluorescent colony relative to the surface, the SNR decreased. At a height of $25 \mu \mathrm{m}$ the signal was barely distinguishable from noise and higher laser powers could not increase the SNR. To ensure reliable particle localization and reduce boundary effects, we limited our analysis to particles with a height from $8 \mu \mathrm{m}$ to $22 \mu \mathrm{m}$ and, which were at least one particle diameter from the colony edge apart 5.6c).

In contrast, particle localization of image stacks acquired with a spinning disk confocal microscope allowed particle localization up to a height of approximately 8 $\mu \mathrm{m}$. This reduced height can be caused by the larger pinhole size of the spinning disk unit. However, when fluorescent and non-fluorescent cells were mixed, the background noise inside the colonies decreased, allowing particle position detection up to a height of $25 \mathrm{\mu m}$.

\subsubsection{Motility and density influence particle tracking}

The successful application of particle tracking algorithms strongly depends on the displacement and the density of particles that should be tracked. In general, particle tracking algorithms link positions of successive frames into trajectories by minimizing the total displacement of the positions. A high particle density combined with a large maximal displacement leads to a very high number of possible combination that are computationally too expensive to calculate. In order to reduce the number of calculations, there are different options.

The first option is to keep the particle displacement low by having a high temporal resolution. This solution is limited by the toxicity of the laser light to bacterial cells during fluorescence microscopy. Phototoxicity can be reduced by decreasing the laser power or the exposure time, where the minimal exposure time is obtained by acquisition of a single image slice. Three dimensional images consist 

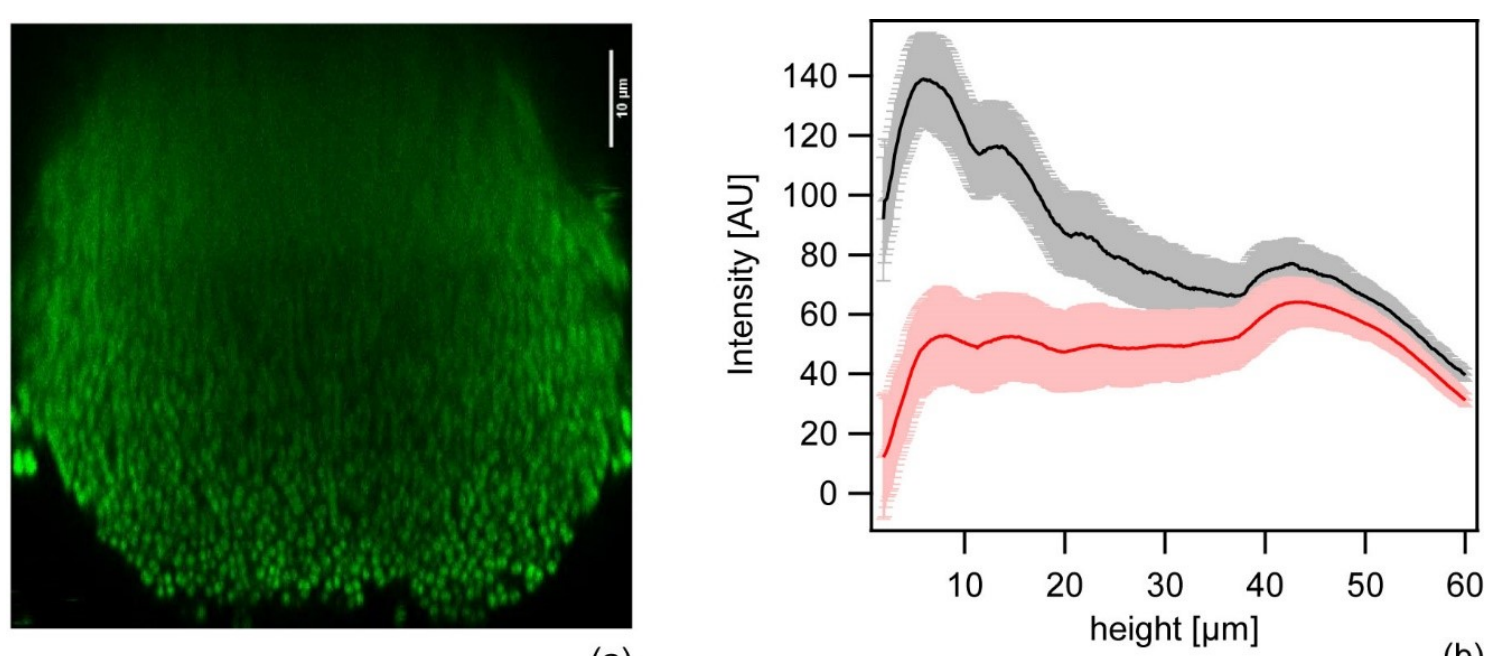

(a)

(b)

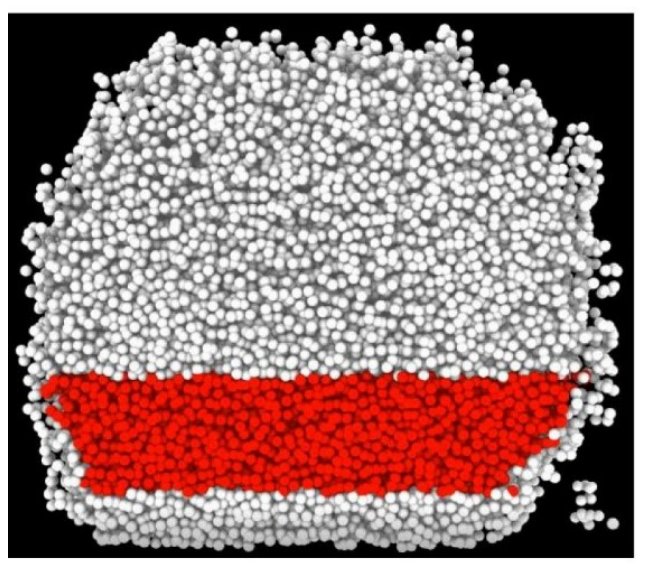

(c)

Figure 5.6: Determination of analysed segment. (a) Cross section of an example stack of a whole $w t$ colony after $24 \mathrm{~h}$. Scale bar: $10 \mu \mathrm{m}$ (b) The intensity of signal (black) and background noise (red) depends on the acquisition height. Mean and standard deviation of signal and noise. Signal and noise were determined separately for each layer from the example stack. The image signal was defined with a mask around the tracked bacterial centroids and the noise was defined as the signal between the bacterial bodies. (c) Tracked bacterial position from example stack are illustrated with white spheres (diameter $=0.7 \mu \mathrm{m})$. Selected bacterial positions for analysis are color coded in red. Image adapted from [44].

typically of several image slices and consequently require a higher exposure time 
depending on their height or their number of image slices. The second option is to keep the particle density low. This solution can be also successfully applied to two dimensional particle tracking, where only neighbors in the focal plane are considered. Alternatively, fluorescent and non-fluorescent cells can be mixed to reduce the density of the fluorescent cells. 


\subsection{Data analysis}

\subsubsection{Characterization of colony structure}

The colony structure was characterized by both the RDF and the remoteness distribution function. Every experimental condition was performed three times and for each experiment 20 image stacks of different colonies were acquired. The obtained RDFs, $g(r)$, were reminiscent of RDFs from Monte Carlo simulations of hard spheres with Lennard-Jones interactions [181, 182]. Since, analytical expressions for the RDF of Lennard-Jones fluids exist, we applied the formula proposed by Matteoli and Mansoori [182],

$$
\left.g\left(r>r_{0}\right)=1+y^{-m}\left[g\left(r_{0}-1-\lambda\right)\right]+\left(\frac{y-1+\lambda}{y}\right)\right) * \exp [-\alpha(y-1)] \cos [\beta(y-1)]
$$

where $r_{0}$ is the nearest neighbor distance, $y=\frac{r}{r_{0}}$ is a normalized variable, and $m, \lambda, \alpha$ and $\beta$ are adjustable parameters. Using equation 5.6, we determined the nearest neighbor distance $r_{0}$ and the corresponding height, which is a measure for order.

The RDF does not provide information about local irregularities inside the colony structure. To address the question whether holes are present within the colony, we implemented the remoteness distribution function [183] that gives the probability of finding a voxel inside the colony and a centroid of a particle a distance $r$ apart. It was computed to measure the size of defects or inhomogeneity inside our bacterial colonies. At first, we distinguished between colony volume and background by thresholding all image stacks to obtain binary image stacks. Thus, the colony volume was one and the background and holes inside the colony were zero. Subsequently, we extruded $4.2 \mu \mathrm{m}$ from the colony edge to remove any artifacts and filled the holes inside all binary images. The next step was computationally very intensive, because we calculated the distance between every colony voxel to the closest particle. As a result, voxels located in previously mentioned 'hole', show larger distances compared to other positions. 


\subsubsection{Growth rate determination}

To determine time-resolved growth rates, we tracked the coordinates of clusters in $1.5 \mathrm{~h}$ intervals $[t, t+1.5 h]$ with $t \in[0 h: 0.25 h: 3 h]$. Additionally, we sorted them into position intervals $\left[r_{e}, r_{e}+2 \mu m\right]$ relative to their mean distance to the edge of colonies $r_{e}$ within $1 \mu \mathrm{m}$ steps. In the following, two different methods will be introduced that can be used to calculate spatio-temporal growth rates inside gonococcal colonies. Please note, that the cell counting method measures growth rates relative to the distance to the colonies edge $r_{e}$, but the growth rates derived from the velocity field are relative to the COM of colonies $r_{c}$. For a better comparison between these methods, we discarded colonies at time $t$, which showed a larger or smaller radius than the standard deviation from the mean colony radius at time $t$.

\section{Determination of growth rate with cell counting (CC method)}

Sfgfp-expressing cells were mixed with non-fluorescent cells in a ratio of 1:100. These fluorescent cells were homogeneously mixed inside non-fluorescent cell dominated gonococcal colonies and formed with progressing time, fluorescent clusters. The positions of clusters were tracked using the particle tracking program written by Y. Gao and M. Kilfoi [143]. The number of cells inside clusters at a specific time interval and mean distance to the edge of colonies were normalized by their initial number of cells $N_{\text {cluster }}(t+\tau) / N_{\text {cluster }}(t)$ and averaged. When at least 5 or 10 clusters fulfilled the conditions, we fitted an exponential function $N_{\text {cluster }}(t+\tau) / N_{\text {cluster }}(t)=\exp (\lambda \tau)$ to the averaged and normalized number of cells to obtain growth rates $\lambda\left(r_{e}, t\right)$.

\section{Determination of growth rate from velocity fields (VF method)}

Here, we aim at measuring growth rates based on the flow profile within colonies. At first, the velocity field had to be obtained from trajectories of clusters. Only the radial component relative to the COM of colonies was of interest. Hence, each trajectory was projected to spherical coordinates to obtain the radial distance $r_{c}=\overrightarrow{r_{c}} \overrightarrow{e_{r}}$, which gives directly the distance between the COM of colonies and the positions of clusters (Fig 5.8). 

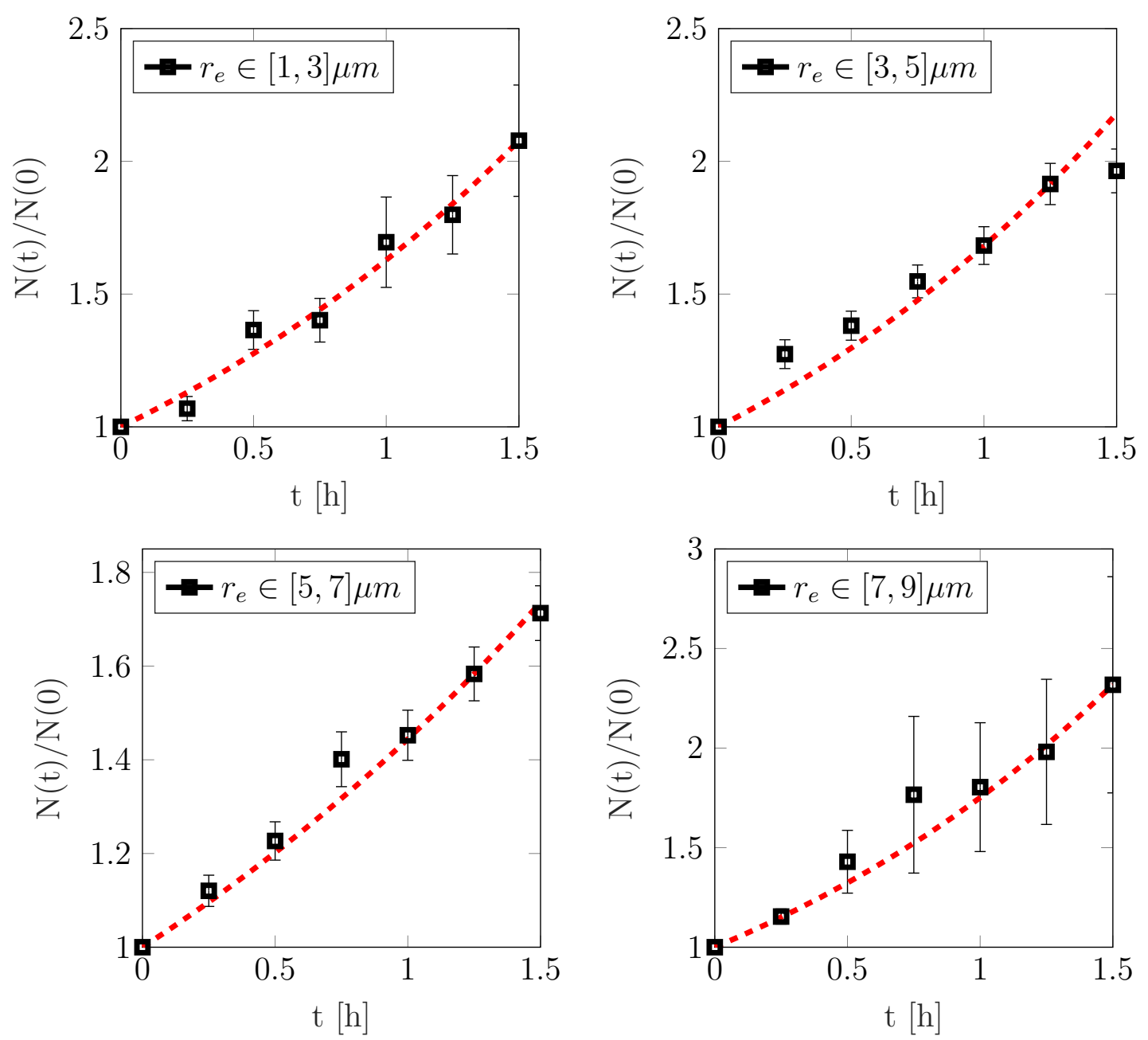

Figure 5.7: Examples of various exponential fits to the normalized number of cells. Red dotted line: exponential fit, black squares: mean normalized number of cells at a given distance from the edge of colonies.

For each time interval $[t, t+1.5 h]$ with $t \in[0 h: 0.25 h: 3 h]$, the velocity of clusters $v_{\text {cluster }}$ was determined by a linear fit $r_{c}(\tau)=v_{\text {cluster }} * \tau+r_{c}(0)$ to the radial distances. The position of clusters was calculated by averaging the corresponding radial distances and sorting them into position intervals $\left[r_{c}, r_{c}+2 \mu m\right]$ within $1 \mu \mathrm{m}$ steps. In order to calculate growth rates from the velocity fields, the continuity equation $\dot{\rho}=-\vec{\nabla} \cdot(\vec{v} \rho)+\lambda \rho$ was solved by Marc Hennes assuming a constant density $\rho$. The velocity fields were determined relative to the COM of colonies $r_{c}$, whereas growth rates determined by the velocity field were plotted 
relative to the mean radius of colonies for a better comparison with the CC method.

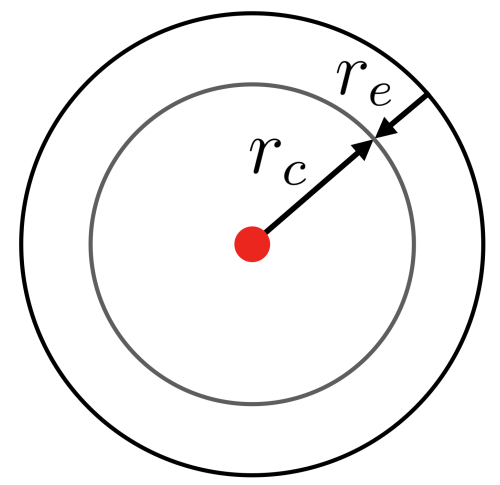

Figure 5.8: Illustration of distance to the edge or COM of colonies. The blue circle has the distance $r_{c}$ to the COM of the colony and the distance $r_{e}$ to the edge of the colony. Red dot: COM of the colony, black: edge of the colony.

To confirm a constant density inside colonies, we generated colonies that contained solely sfgfp fluorescent cells (Fig. 5.9a). Using the spinning disk confocal microscope, we could access only bacterial positions below an acquisition height of $8 \mu \mathrm{m}$ inside colonies. Optimally, the density had to be determined relative to the COM of colonies. But due to the height restriction, the amount of data needed to determine the density decreased rapidly for larger distances from the edge of colonies. This issue was solved by using both spherical and cylindrical coordinates. On the one hand, spherical coordinates gave the spatial density distribution up to a distance of $4 \mu \mathrm{m}$ from the edge of colonies. On the other hand, cylindrical coordinates allowed density determination up to a distance of $16 \mu \mathrm{m}$ from the edge relative to the z-axis of the $\mathrm{COM}$ of colonies. Both methods yielded a comparable results indicating a homogeneous density distribution throughout the colony. At a distance of $r_{c}<3 \mathrm{~cm}$ from the edge of the colony, the density decreased (Fig. 5.9b). This inhomogeneity of cell density has to be take into account when determining local growth rates. 

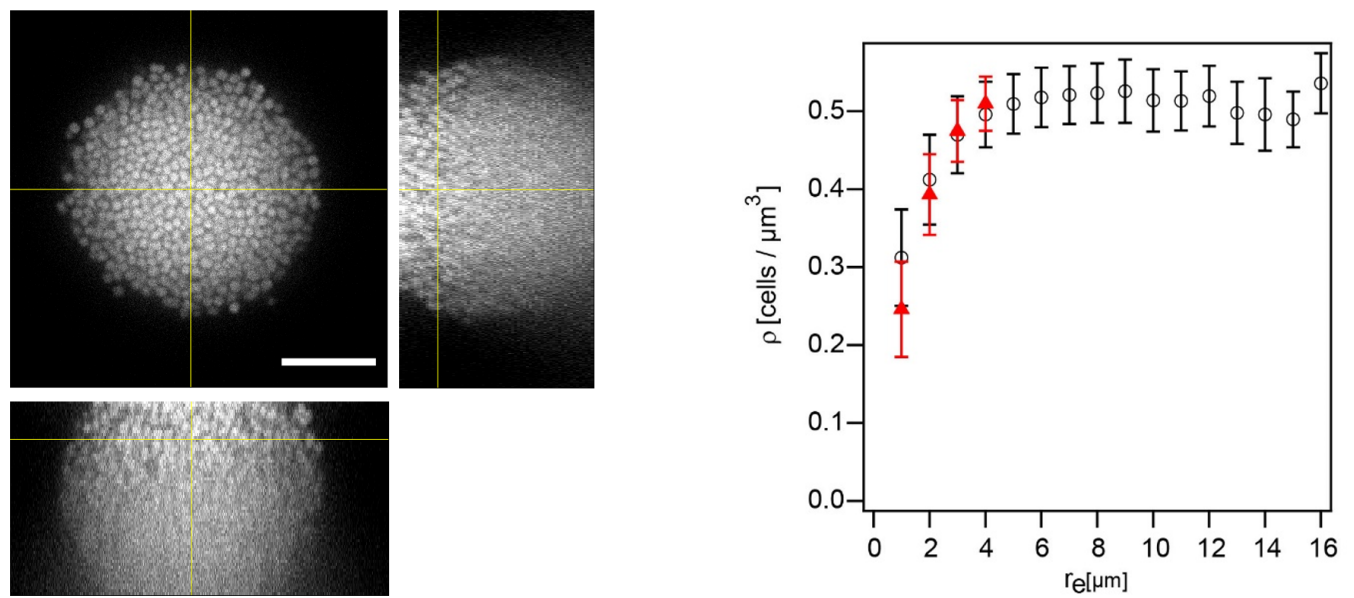

(a)

(b)

Figure 5.9: a) Typical orthogonal view of wt green (Ng 194) colonies formed after $1 \mathrm{~h}$. Scale bar $10 \mu \mathrm{m}$. b) Cell density profile through colony. Black circles: cylindric coordinates, red triangles: spherical coordinates. Image adapted from [172].

\subsubsection{Determination of fraction of dead cells}

Here, we aimed to determine the fractions of dead cells locally. To visualize dead cells, we used a DNA intercalating dye that penetrates the cell envelope of dead but not of living cells. To this end, we stained all colonies with PI, which was supplemented to the growth medium and constantly supplied into our flow chambers. The positions of dead cells inside colonies were located using the same method, which was used to locate living cells (Chap. 5.4.3). The number of dead cells were binned to $2 \mu \mathrm{m}$ intervals relative to the edge of the colony and divided by the total number of cells located in the corresponding volume section $\delta^{*}(r)=\frac{\delta(r)}{\rho(r) V(r)}$, where $\delta(r)$ was the number of dead cells inside the volume section $V(r)$ and $\rho(r)$ was the total cell density. The cell density was assumed to be constant. This assumption was false for distances smaller than $3 \mu \mathrm{m}$ away from the edge of colonies. Thus, the fraction of dead cell was underestimated there. For a better comparison between the fraction of dead cells and the growth profiles obtained by CC and VF method, we averaged the fraction of dead cells inside time intervals of $[t, t+1.5 h]$. 


\section{Results}

In this thesis, we investigated the architecture and growth in gonococcal colonies in space and time. In the first section, we characterize the structure of fixed colonies. In particular, we are interested in how T4P motor activity affects the local cell density and local order of colonies. We generated different mutant strains and characterized their T4P motor activity and T4P-T4P interactions. Subsequently, we investigated structures of colonies formed by mutants strains via RDF and remoteness distribution function after $6 \mathrm{~h}$ and $24 \mathrm{~h}$ of growth.

In the second section, we developed novel methods that enable us to determine spatio-temporal growth rates of living gonococcal colonies via counting the offspring of individual cells or characterizing the velocity field. Both methods have their advantages and disadvantages that can be combined and utilized for a robust growth rate characterization. In order to to study how the system responds to perturbations, we applied different stresses and interfered with a stress response system. 


\subsection{Structural properties of gonococcal colonies}

Using laser scanning confocal microscopy, we were able to characterize the structure of fixed gonococcal colonies with the RDF and the remoteness distribution function. Considering that gonococcal colonies are spherical and fuse upon contact, we hypothesized that they may have properties of liquids. To assess this hypothesis, we investigated the local order of the colonies. Furthermore, we addressed the question whether the T4P motor properties affect the materials properties of the colonies.

\subsubsection{Gonococcal colonies show liquid-like order}

We hypothesized that the gonococcal T4P machinery supports local ordering and generates a liquid like colony structure. Gonococci were allowed to grow into colonies for $6 \mathrm{~h}$ and $24 \mathrm{~h}$, respectively, before they were fixed and imaged as described in the methods. To investigate local order, we determined the RDF. For liquids, we expect short-ranged order, for solids long-ranged order, and for gas, we expect a flat RDF. For distances larger than $2.5 \mu \mathrm{m}$, which corresponds to approximately three cellular diameters, the RDF determined from colonies formed by the $w t$ (Fig. 6.1a,b) converged to one (Fig. 6.1c). However, for lower distances, we observed two peaks. The first peak, which can be interpreted as the nearest neighbor distance, is higher compared to the second peak, which can be consequently interpreted as the second nearest neighbor distance. The higher the amplitude of the first peak $g\left(r_{0}\right)$, the higher is the order and vise versa. We found that the nearest neighbor distance decreased from $r_{0}^{6 h}=(1.18 \pm 0.02) \mu m$ to $r_{0}^{24 h}=(1.14 \pm 0.01) \mu m$ and the amplitude increased from $g\left(r_{0}\right)^{6 h}=(1.23 \pm 0.02) \mu m$ to $g\left(r_{0}\right)^{24 h}=(1.46 \pm 0.02) \mu m$ as a function of time. In summary, local order and density increase with time for gonococcal colonies.

\subsubsection{Motor activity accelerates local-ordering}

T4P are important for regulation of structure and dynamics of biofilms [36, 184, $37,185]$. To address the question, which role T4P retraction plays for the structure of gonococcal colonies, we generated mutant strains with modified T4P motor 

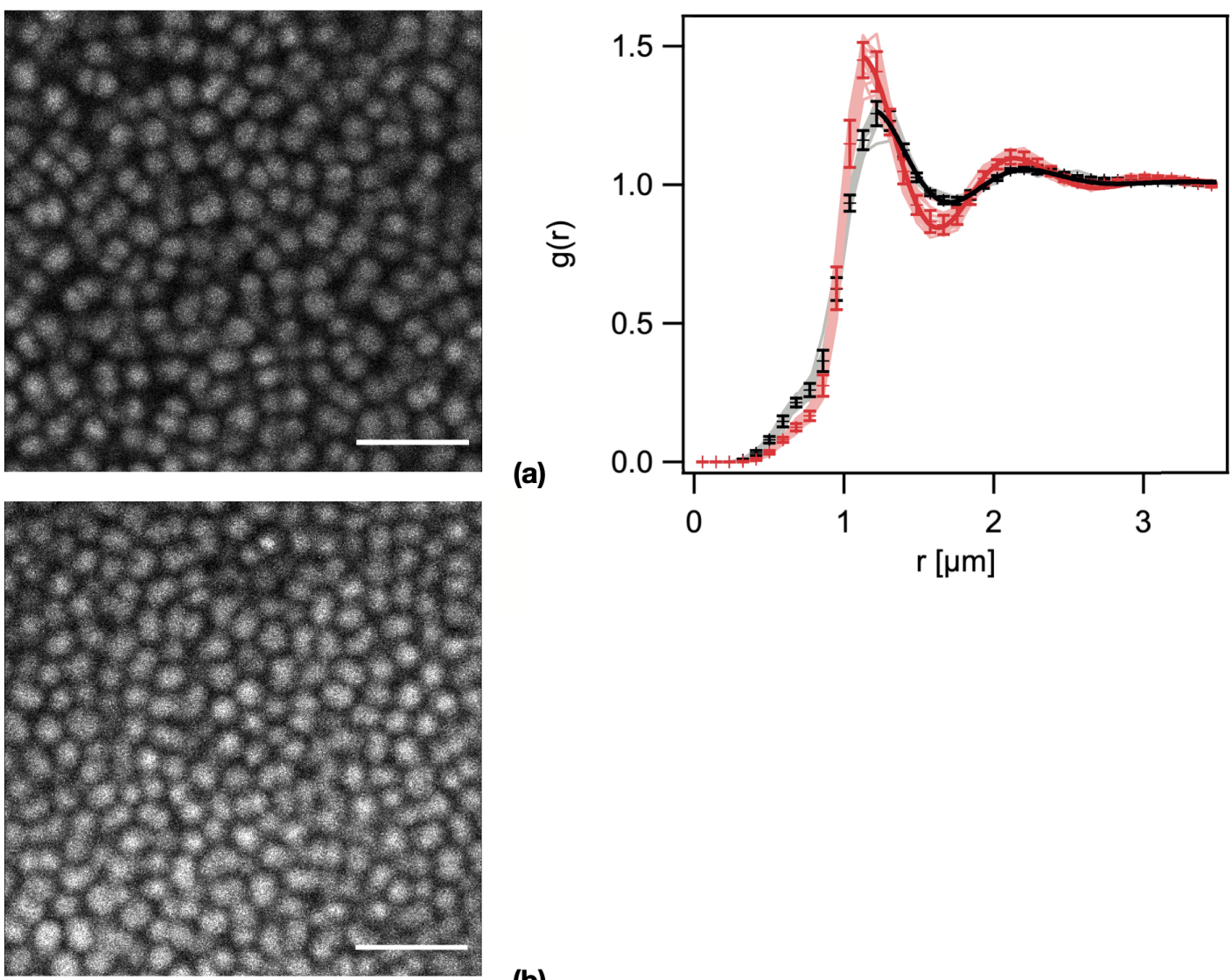

(c)

(b)

Figure 6.1: Liquid-like order of gonococcal $w t$ colonies. Confocal section of $w t$ colonies after (a) $6 \mathrm{~h}$ and (b) $24 \mathrm{~h}$ of growth. Scale bar: $4 \mu \mathrm{m}$. RDF after $6 \mathrm{~h}$ (black) and $24 \mathrm{~h}$ (red). Shaded lines: RDF of individual colonies, full lines: fit to equation 5.6. Error bars contain mean values and standard deviations of 15 colonies from three independent experiments. Image adapted from [44] and reproduced with permission.

activity. Our assumption was that cells inside colonies form a T4P network, where pili bind to each other, followed by retraction events that cause their bonds to rupture (Fig. 6.2). This tug of war mechanism would allow cell motility and reorganization of cells within colonies. 

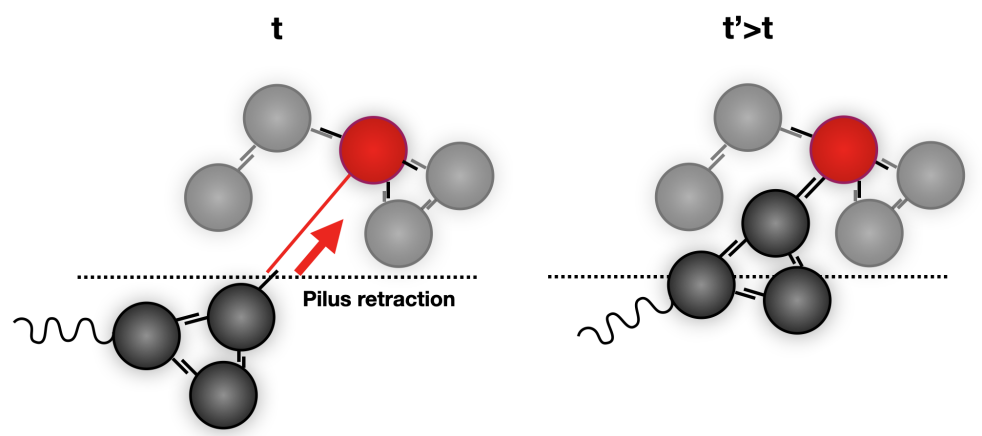

Figure 6.2: Sketch of how T4P interaction between adjacent cells mediate cell re-organization. The pilus of the red cell binds to a close cell aggregate and minimizes the distance of both aggregates through pilus retraction.

To investigate how intermediate phenotypes between full T4P retraction activity and low T4P retraction activity affect colony structure, we generated two $\Delta p i l T_{W B}$ mutant strains, which express both functional and non-functional PilT. PilT forms hexamers prior to binding to the T4P complex to induce pilus retraction [49]. For this project, I teamed up with Tom Cronenberg and Robert Zöllner to correlate T4P motor activity, T4P-mediated attractive interactions and local structure of colonies.

By expressing both functional and non-functional PilT at different ratios, most likely heterohexamers are formed. Tom Cronenberg characterized the effect of $\Delta p i l T_{W B}$ expression on T4P motor activity. The presence of non-functional PilT induced lower retraction velocities and a higher probability of finding pili in the state of pilus elongation or pausing. Next to the effect on motor properties, it was important to characterize the effect of $\Delta p i l T_{W B}$ expression on attractive interactions between gonococci. Robert Zöllner used a double-laser trap to measure T4P-mediated attractive interactions. Interestingly, the presence of non-functional PilT did not influence the rupture force of T4P-T4P binding, but the transition frequency at different states like retraction, elongation and pausing changed. In particular, the T4P-T4P bond rupture and retraction frequency decreased for $\Delta p i l T_{W B}$ strains, while elongation and pausing frequencies increased. As a result, the probability that two cells are attached to each other increases for $\Delta p i l T_{W B 1}$ strains and even more for $\Delta$ pilT $T_{W B 2}[44]$. 
Additionally, we generated the $\Delta$ pilT mutant strain lacking the T4P retraction ATPase PilT. In the absence of PilT, slow retraction velocities were reported indicating that PilT is not essential for pilus retraction. However, retraction velocities and force generation without the retraction ATPase were too low to support twitching motility or fluidization of colonies [27]. Typically, gonococcal colonies are spherically shaped (Fig. 6.3a,d). While the presence of non-functional PilT still supports the spherical shape of colonies (Fig. 6.3b,e), the $\Delta$ pilT mutant strain does not form spherical colonies [186, 185] (Fig. 6.3c,e), indicating that active force generation is important for colony organization.
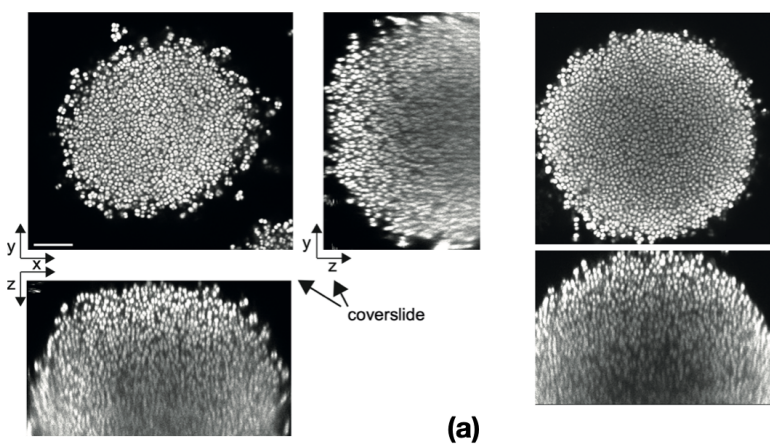

(a)
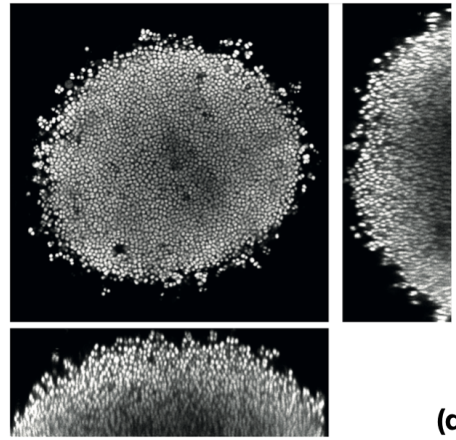

(d)
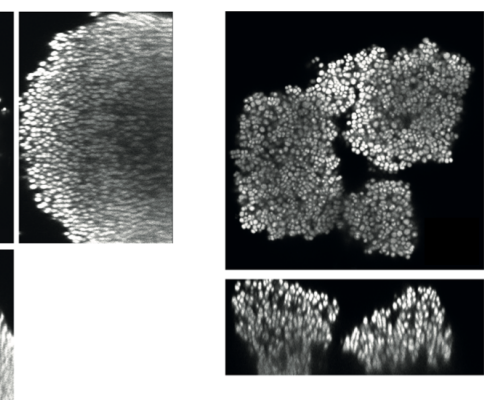

(b)
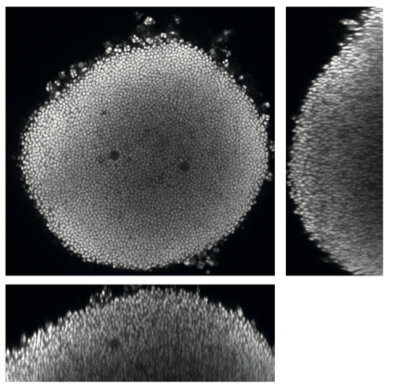

(e)

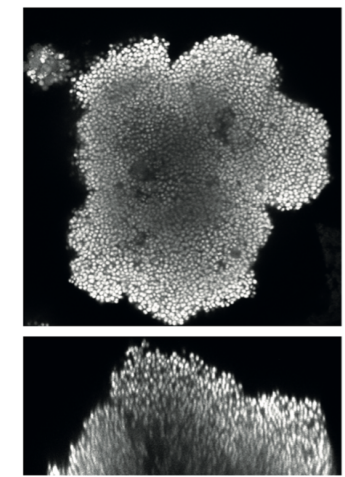

(c)

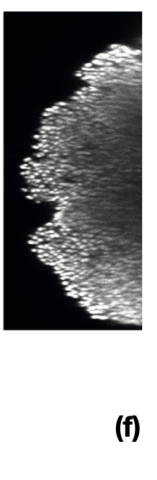

Figure 6.3: Orthogonal views of image stacks of gonococcal colonies with different T4P motor properties. Typical colonies of (a) wt, (b) $\Delta p i l T_{W B 2}$ and (c) $\Delta$ pilT formed after $6 \mathrm{~h}$ and $(\mathrm{d}, \mathrm{e}, \mathrm{f}) 24 \mathrm{~h})$. Image adapted from [44] and reproduced with permission.

To access the effect of T4P motor activity on the local order of gonococcal 
colonies we computed the local cell density and RDFs. A higher pilus motor activity supports a higher local cell density on shorter time scales (Fig. 6.4g,h,i). For all strains, densities increase with time (Fig. 6.4) and converge to a maximal density of 0.55 cells $/ \mathrm{um}^{3}$. Solely colonies formed by the $\Delta$ pilT mutant strain show a lower cell density after $24 \mathrm{~h}$, indicating that active force generation is necessary to reach high cell densities.
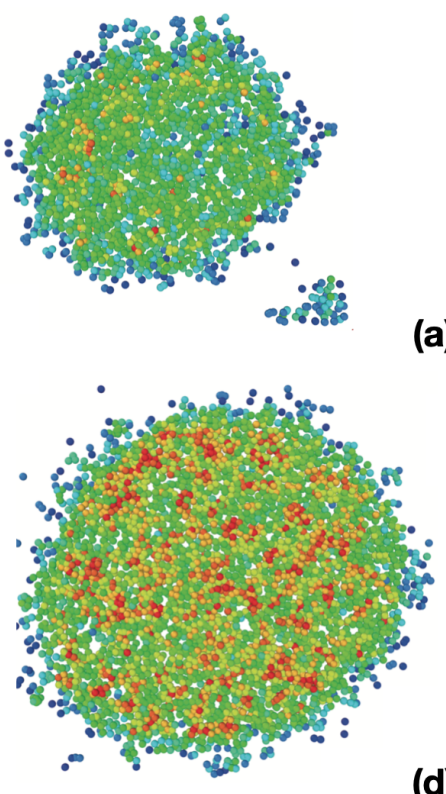

(d)

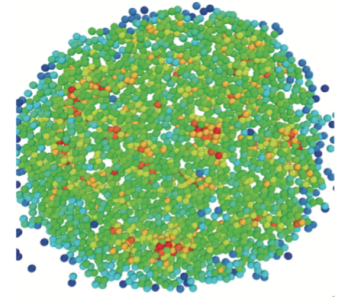

(a)

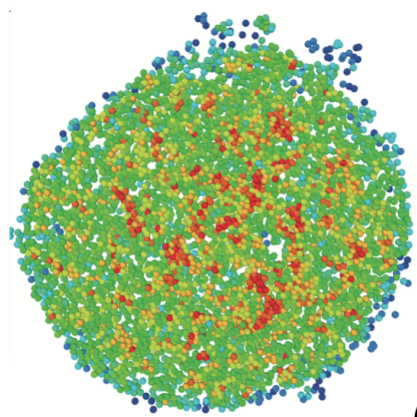

(e)

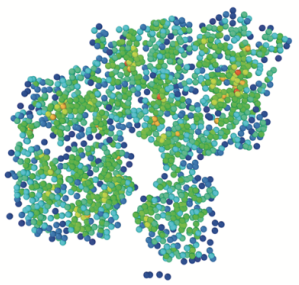

(c)

(b)

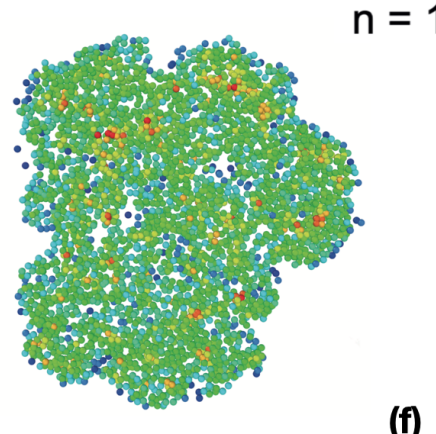

(f)

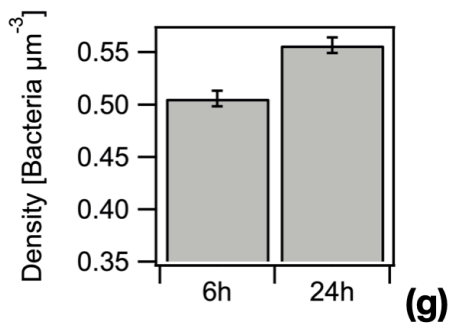

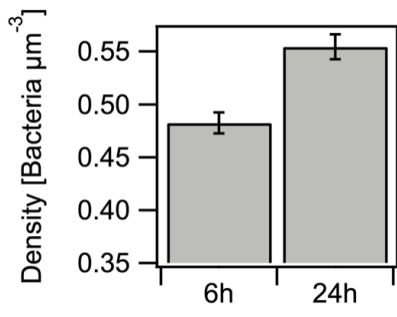

(h)

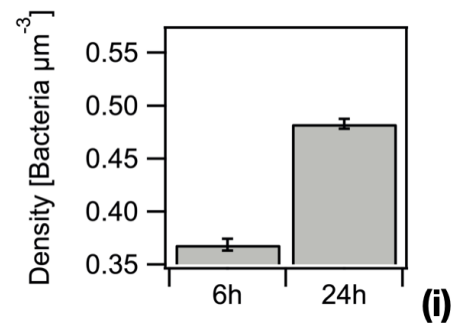

Figure 6.4: Reconstruction of bacterial coordinates of colonies formed by (a) wt, (b) $\Delta p_{i l T} T_{W 2}$ and (c) $\Delta$ pilT after $6 \mathrm{~h}$ and $(\mathrm{d}, \mathrm{e}, \mathrm{f}) 24 \mathrm{~h}$. The colors encode the number of nearest neighbors $n$. Local cell density of colonies formed by (g) $w t$, (h) $\Delta p i l T_{W B 2}$ and (i) $\Delta p i l T$ after $6 \mathrm{~h}$ and $24 \mathrm{~h}$. Error bars: standard errors of 15 colonies from three independent experiments. Image adapted from [44] and reproduced with permission. 
The RDF of colonies formed by the $\Delta p i l T_{W B 2}$ strain show a short-ranged order similar to the $w t$ (Fig. 6.5a), but the nearest neighbor distance (Fig. 6.5c) and the amplitude of the first RDF peak (Fig. 6.5d) are higher, confirming the lower local cell density (Fig. 6.4g,h) and indicating more local order. After $24 \mathrm{~h}$, RDF of colonies formed by the $\triangle p i l T_{W B 2}$ strain shows a neighbor distance of $r_{0}^{W B 2,24 h}=(1.13 \pm 0.01) \mu m$ and an amplitude of $g\left(r_{0}\right)^{W B 2,24 h}=(1.48 \pm 0.02) \mu m$, which is comparable with the RDF obtained from wt colonies. $\Delta$ pilT colonies do not show maxima in their RDF, indicating a gas-like arrangement after $6 \mathrm{~h}$ of colony growth (Fig. 6.5 b). After 24 h, local order emerged and the nearest neighbor distance decreased. Probably, cells were able to generate forces during
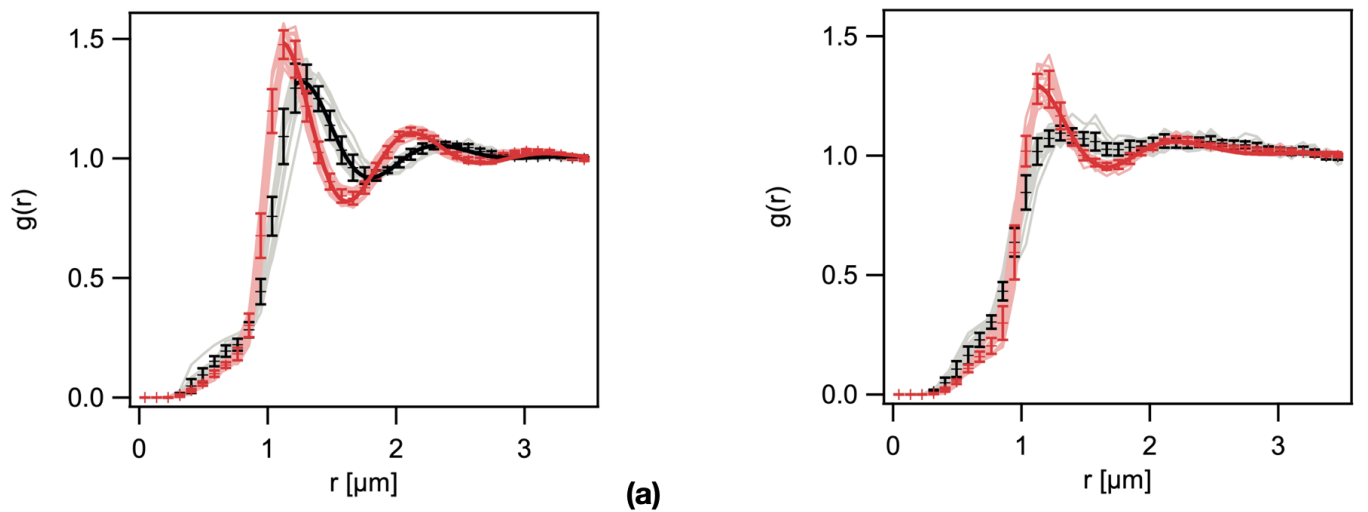

(a)

(b)
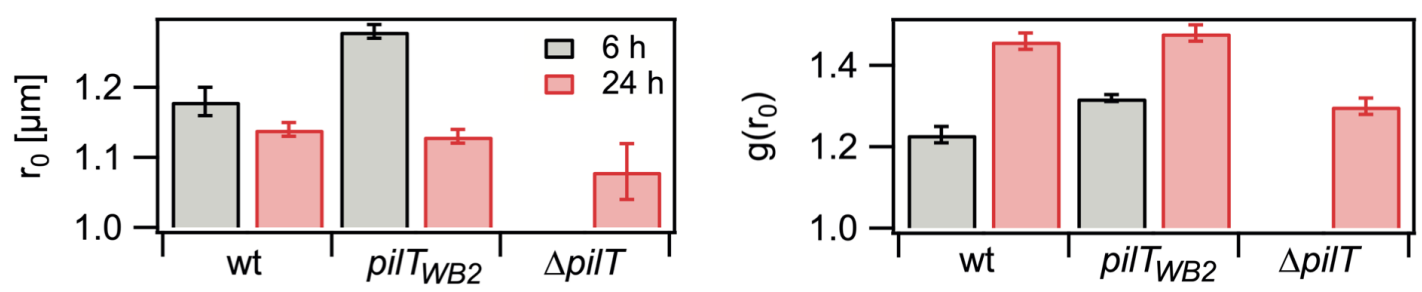

(c)

Figure 6.5: Comparison of RDFs of colonies formed by (a) $\Delta p i l T_{W B 2}$ and (b) $\Delta p i l T$ after $6 \mathrm{~h}$ (black) and $24 \mathrm{~h}$ (red). Shaded lines: RDF of individual colonies, full lines: fit to equation 5.6. Error bars contain mean values and standard deviations of 15 colonies from three independent experiments. (c) Contact distance $r_{0}$ and (d) value of RDF at $r_{0}$ obtained from fit to Eq. 5.6. Image adapted from [44] and reproduced with permission. 
growth through volume exclusion or simply filled the free space through growth. Additionally, low force generation can be sufficient for bacterial rearrangements at long time scales. In conclusion, T4P force generation is not essential for emergence of local order, but it accelerates the process, causing higher cell densities at earlier stages.

\subsubsection{Gonococcal colonies show defects in their structure}

After $24 \mathrm{~h}$, we observed holes in the structure of $w t$ and $\Delta p i l T_{W B 2}$ mutant colonies stained by SYTO9 (Fig. 6.3d,e), which have not been there after 6 h (Fig. 6.3a,b). We characterized the holes inside $w t$ colonies by the remoteness distribution [183] The remoteness distribution gives the probability of finding the shortest distance $r$ between a voxel located inside a colony and a position of a cell. Thus, by definition, the maximum of the remoteness distribution (Fig. 6.6) corresponds to half the nearest neighbor distance determined by the RDF (Fig.6.5a,b). We observed that the remoteness distribution shifted to higher values for $24 \mathrm{~h}$ old colonies (Fig. 6.6a,b), which confirmed our observation of more holes inside colonies after $24 \mathrm{~h}$ compared to $6 \mathrm{~h}$. The size of holes inside $w t$ and $\Delta p i l T_{W B 2}$ colonies are similar (Fig.6.5a,b), indicating that motor activity does not influence defects in the structure of gonococcal colonies.

We assumed that these holes were caused by dead cells that still occupied the space, but staining dead cells with PI did not work together with cell fixation. Later, image stacks of wt green colonies were acquired with a spinning disk confocal microscope without cell fixation. Indeed we observed again holes in the colony structure. PI staining confirmed that dead cells were located inside $w t$ green colonies, causing areas without fluorescence. Theoretically, SYTO9 stains both living and dead cells, where the intensity from dead cells should be weaker compared to living cells.

In contrast to spherically shaped colonies, colonies formed by the $\Delta$ pilT strain show large holes on short time scales (Fig. 6.3c). After $24 \mathrm{~h}$, the size of holes inside $\Delta$ pilT colonies decreases (Fig. 6.6c), but the holes are still larger in comparison 
to holes inside $w t$ and $\Delta p i l T_{W B 2}$ colonies. In conclusion, high forces during pilus retraction are necessary to allow cell rearrangement and reduce the size of holes inside gonococcal colonies.
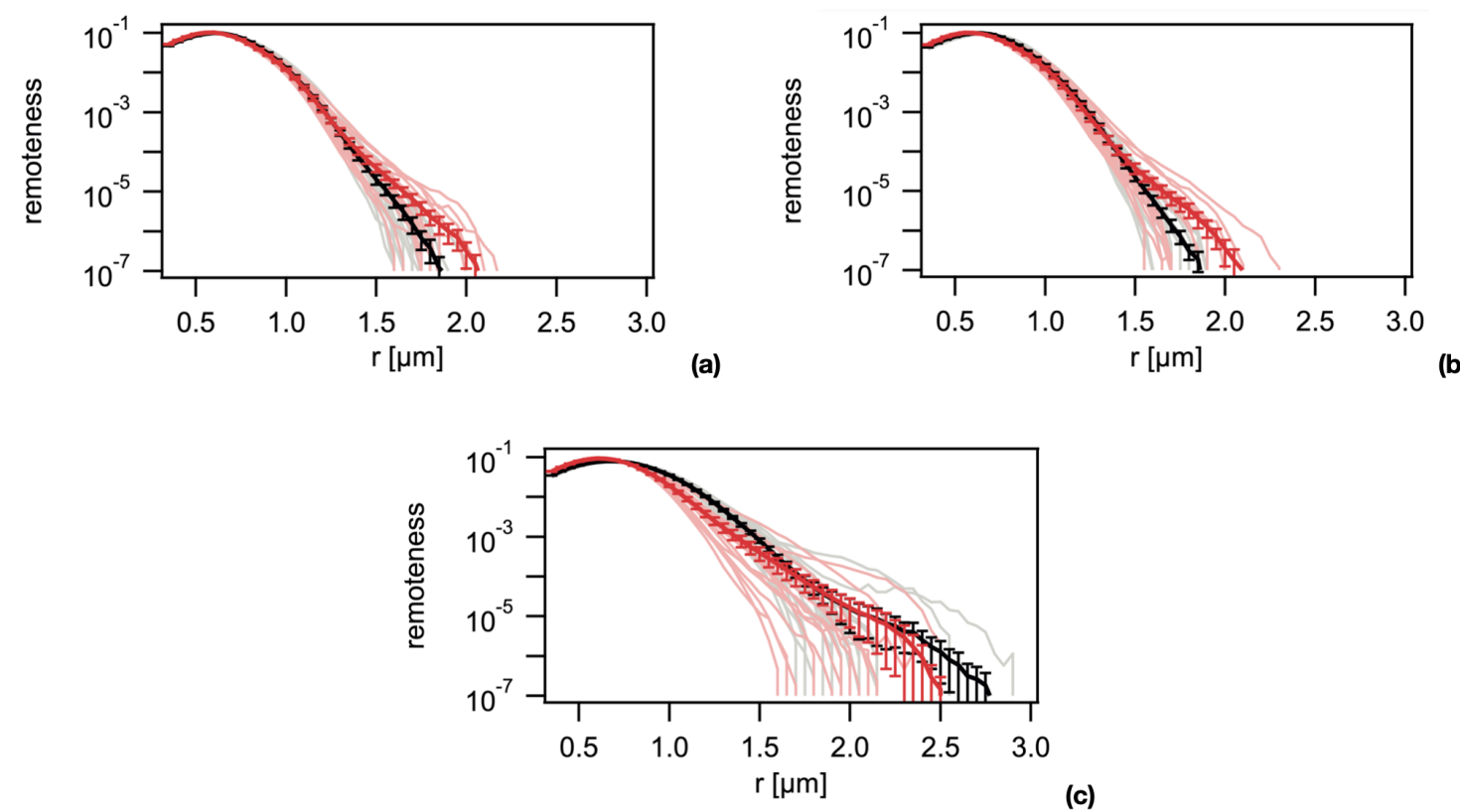

Figure 6.6: Remoteness distribution of colonies formed by (a) wt, (b) $\Delta p i l T_{W B 2}$ and (c) $\Delta$ pilT after $6 \mathrm{~h}$ (black) and $24 \mathrm{~h}$ (red). Error bars contain mean values and standard deviations of 15 colonies from three independent experiments. Image adapted from [44] and reproduced with permission. 


\subsection{Dynamics and growth within bacterial colonies}

Growth of bacteria has been characterized at the single cell level for planktonic bacteria and at the colony level for bacteria residing within colonies. Measuring growth rates at spatial and temporal resolution within colonies remains challenging. Here, we use spinning disk confocal microscopy to characterize spatio-temporal growth and death dynamics within gonococcal colonies. First, we measured the mean growth rate inside gonococcal colonies by estimating the total number of cells from radii of colonies. Subsequently, growth rates were determined with single cell resolution by cell counting (CC method) and from the velocity field (VF method). Since, all methods generated consistent growth rates, we investigated the effect of environmental and chemical changes on the spatio-temporal growth and death dynamics. To this end, we varied the nutrient supply, inhibited stringent response or treated gonococcal colonies with azithromycin.

\subsubsection{Colony growth shows deviations from exponential growth after two hours}

At first, we analyzed the growth of $w t$ colonies by monitoring the radius $R(t)$ of gonococcal colonies for several hours in our flow chamber setup under constant nutrient supply. The cubed colony radius is proportional to the number of cells inside a colony $N_{c o l}(t)=\rho V=4 / 3 \rho \pi R(t)^{3}$. Due to fluctuations of the initial colony size, we normalized $N_{c o l}(t)$ to the initial number of cells $N_{c o l}(0)$. Assuming a constant density, we get $(R(t) / R(0))^{3}=N_{\text {col }}(t) / N \operatorname{col}(0)$. By fitting an exponential function $N_{\text {col }}(t) / N \operatorname{col}(0)=\exp \left(\lambda_{\text {col }} t\right)$ to the first 2 hours, we were able to calculate the mean colony growth rate $\lambda_{c o l}=(0.56 \pm 0.01) h^{-1}$. Afterwards, $N_{\text {col }}(t) / N_{c o l}(0)$ deviated from exponential growth, indicating a transition from homogeneous to heterogeneous growth inside gonococcal colonies (Fig. (6.7)).

In the next step, we repeated the procedure for sfgfp expressing bacteria (wt green) to check for growth rate differences. Indeed, the sfgfp expression under the strong pile promoter caused a lower mean colony growth rate $\lambda_{\text {col }}^{\text {green }}=(0.42 \pm 0.01) h^{-1}$ (Fig. 6.7). In summary, both strains showed the transition from exponential growth 
to sub-exponential growth at the same age. We suggest, that growth rates became inhomogeneous at this point of time. Our hypothesis will be addressed in the following chapters.

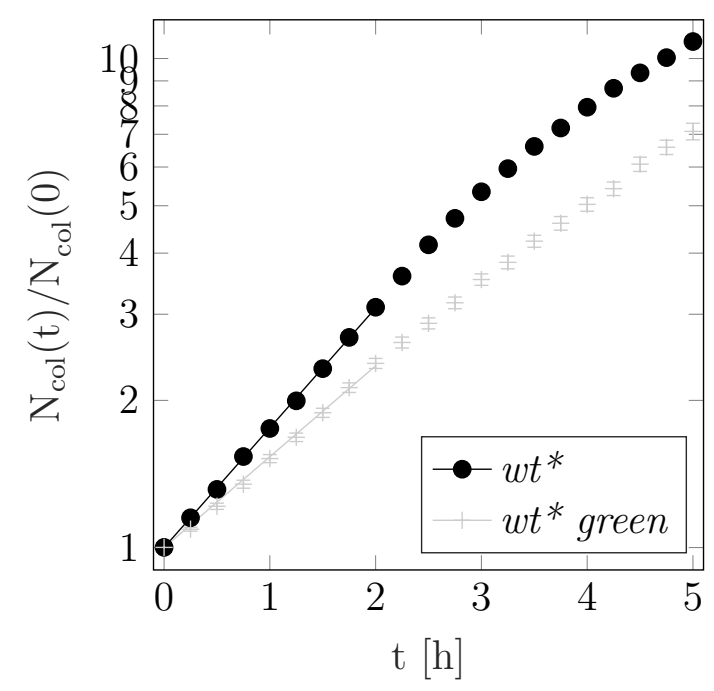

Figure 6.7: Expression of $s f g f p$ reduces growth rate. Mean number of cells within colony $N_{\text {col }}(t)$ normalized by the number of cells in the colony at $t=0, N_{c o l}(0)$ for $w t$ (Ng150, black) and wt green (Ng194, grey). Full lines: exponential fits with $\left(N_{\text {col }}(t)\right) /\left(N_{\text {col }}(0)\right)=\exp \left(\lambda_{\text {colony }} t\right)$. $\lambda_{(\text {colony }, w t)}=(0.563 \pm 0.003) h^{-1}$ and $\lambda_{\text {colony,wtgreen }}=(0.42 \pm 0.01) h^{-1}$. (mean \pm se, $>40$ colonies for each data point). Image adapted from [172].

\subsubsection{Lineage tracking within gonococcal colonies reveals development of growth profiles}

We developed a method to track lineages inside gonococcal colonies. At first, we tried to track all cells inside our colonies, but we observed a low penetration depth of only 8 um due to background noise. Furthermore, whole colonies as well as single cells within colonies were motile. As a result, the particle tracking program from Y. Gao and M. Kilfoil failed to track all cells inside our densely packed colonies. Setting a low maximal displacement in the particle tracking program gave only a fraction of single cell trajectories, because some cells had a larger displacement. 
raw data
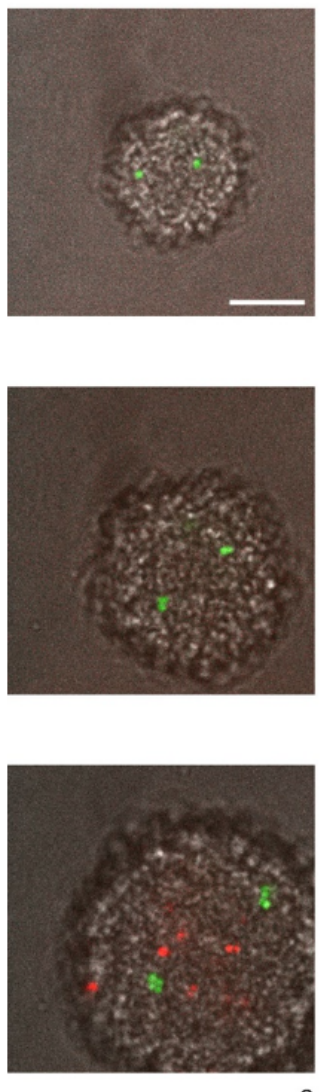

detection of single cell positions
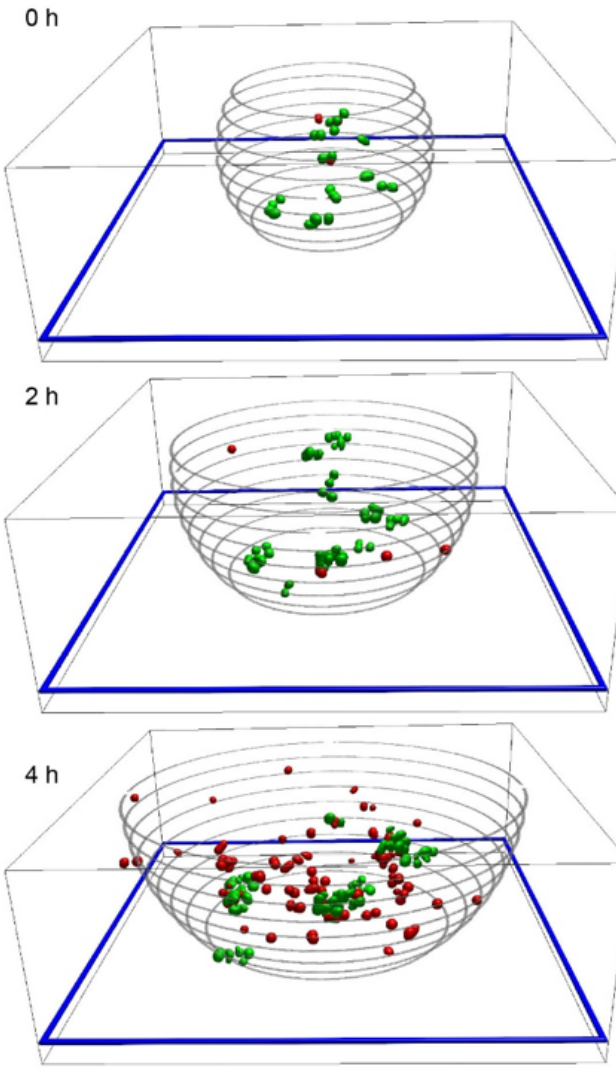

cluster analysis

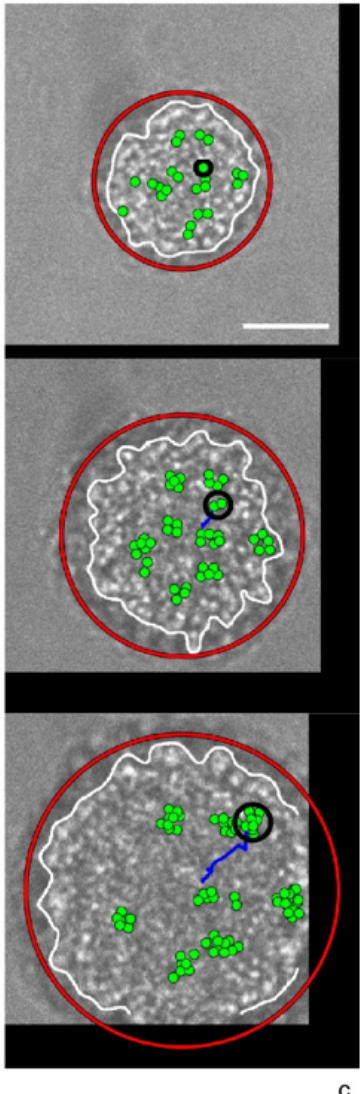

Figure 6.8: Lineage tracking in gonococcal colonies. wt (Ng150) mixed with wt green (Ng194). a) Typical confocal plane $(\mathrm{h}=3 \mu \mathrm{m})$ through a colony at different time points. Overlay between brightfield (grey), sfGFP fluorescence (green), and PI fluorescence (red). b) 3D reconstruction of positions of fluorescent cells. The blue frame denotes the plane shown in a). $\Delta h$ between black circles: $1 \mu \mathrm{m}$. c) Cluster dynamics in growing colony. Green circles: Positions of wt green gonococci. 3D projections of all wt green cells are shown. Black circle: Circumference of single cluster. Blue line: Trajectory of centre of mass of cluster. White line: Edge of colony. Red circle: Circle depicting radius of colony. Scale bars: $10 \mu \mathrm{m}$. Image adapted from [172]

Increasing the maximal displacement value lead to failure of the particle tracking program as a consequence of too many possible combinations. Theoretically, we could have increased the time resolution to minimize the displacement of cells 
between to successive image stacks, but this was prohibited by the toxicity of the laser light to our sample, which limited our time resolution to $15 \mathrm{~min}$. Unfortunately, the division plane of gonococci switches every generation [187] and due to the low spatio-temporal resolution monitoring growth from gonococci to diplococci was not possible.

In order to reduce the density of fluorescent cells, we successfully mixed fluorescent and non-fluorescent cells at a ratio of 1 : 100. In freshly assembled colonies we observed that the small sub-population of single fluorescent cells were surrounded by non-fluorescent neighbors. Later, the fluorescent cells divided and formed larger fluorescent clusters, which were defined as clusters (Fig. 6.8). In the following two sections, two different methods will be described that were used to calculate spatio-temporal growth rates from the cluster tracking. In the first method we count the number of cells associated with a specific cluster and fit an exponential function to calculate the growth rate. The second method takes the displacement of clusters into account. Growing cells within dense colonies generate pressure on their neighbors, leading to a velocity field that points from the center of mass of a colony to its edge. Thus, the velocity field gives information about the growth dynamics of cells inside a spherical colony (Fig. 6.13c), where faster growing cells generate steeper velocity gradients and vice versa.

\section{Cell counting (CC) method reveals heterogeneous growth profiles}

The CC method determines the effective growth rate of the fluorescent wt green cells inside a colony consisting mainly of non-fluorescent $w t$ cells. Young colonies typically contained only a few fluorescent cells that were surrounded with nonfluorescent neighbors. These cells and their offspring were assigned to clusters (Fig. 6.9a). By tracking the COM of clusters, we were able to count the number of cells inside each corresponding cluster with a temporal resolution of $15 \mathrm{~min}$. Clusters that started with a single cell showed almost discrete time steps with a generation time of around $1.5 \mathrm{~h}$ (Fig. 6.9b). The almost simultaneous cell divisions occurred, because the offspring resided within the same stage of the cell cycle. In contrast, when a cluster was initiated by multiple cells, their cell cycles were not 
synchronized. Consequently, cell divisions occurred not correlated in time.

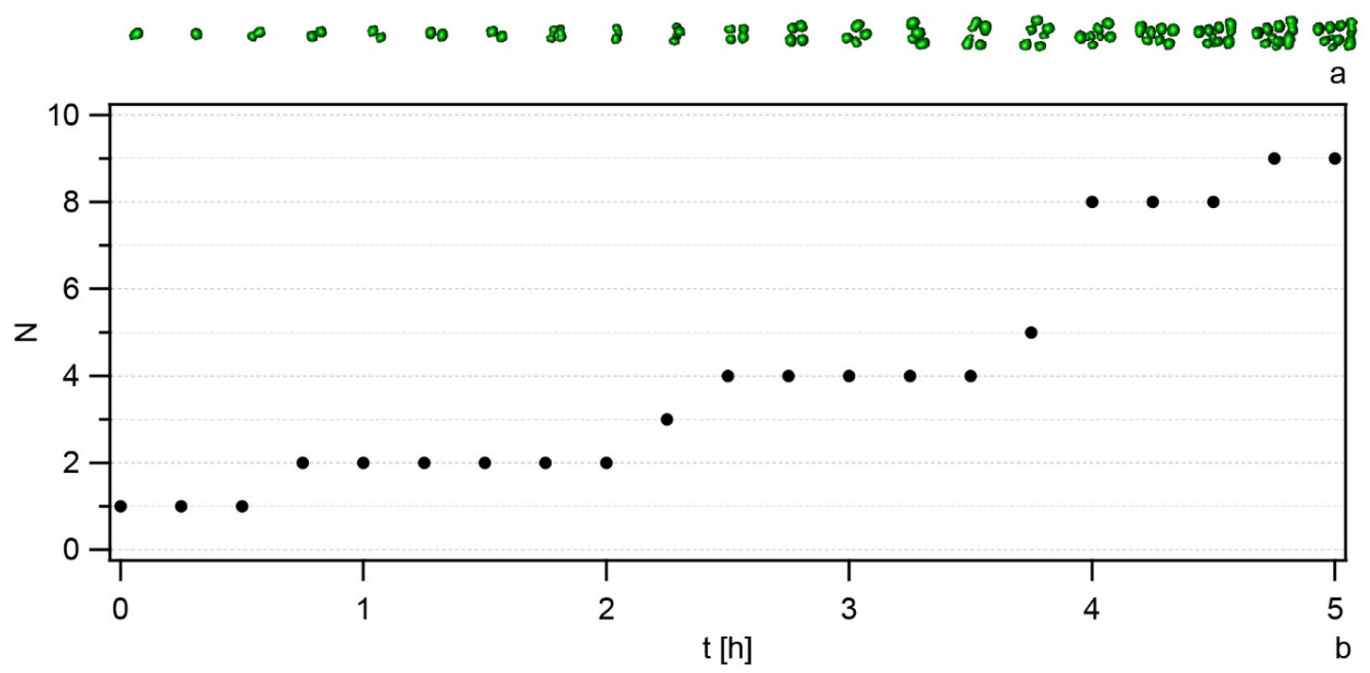

Figure 6.9: a) Example of single growing cluster highlighted in Fig. 6.8. b) Number of cells $N(t)$ within the cluster shown in a). Image adapted from [172]

In general, cells divide with a growth rate $\lambda$ and die with a death rate $\delta$. They follow the differential equation $\dot{N}=\frac{d N}{d t}=(\lambda-\delta) N$, where $x$ is the number of cells. The corresponding solution is an exponential function $N(t) / N_{0}=e^{\lambda_{\text {eff }} t}$ with $\lambda_{e f f}=\lambda-\delta$ and an initial cell number of $N_{0}$.

By splitting the data into $1.5 \mathrm{~h}$ long time intervals $[t, t+1.5]$ and sorting them relative to the colony edge in position intervals $\left[r_{e}, r_{e}+2\right]$, we assigned spatiotemporal growth rates to each cluster by fitting an exponential function (Eq. 6.1) to each time and position interval (Fig. 6.10a). Additionally, the fraction of dead cells in the corresponding position and time intervals were determined (Fig. 6.10b).

$$
N(t+\tau) / N(t)=\exp (\lambda * \tau)
$$

During the initial $2 \mathrm{~h}$, we observed a homogeneous growth profile throughout the colony with a growth rate of $\lambda_{w t}=(0.42 \pm 0.03) h^{-1}$ (Fig. 6.10c). Afterwards, growth rates decreased towards the colony COM. This observation is consistent with the transition from exponential to sub-exponential growth, calculated from the radii of colonies. The fraction of dead cells was maximal around the COM 


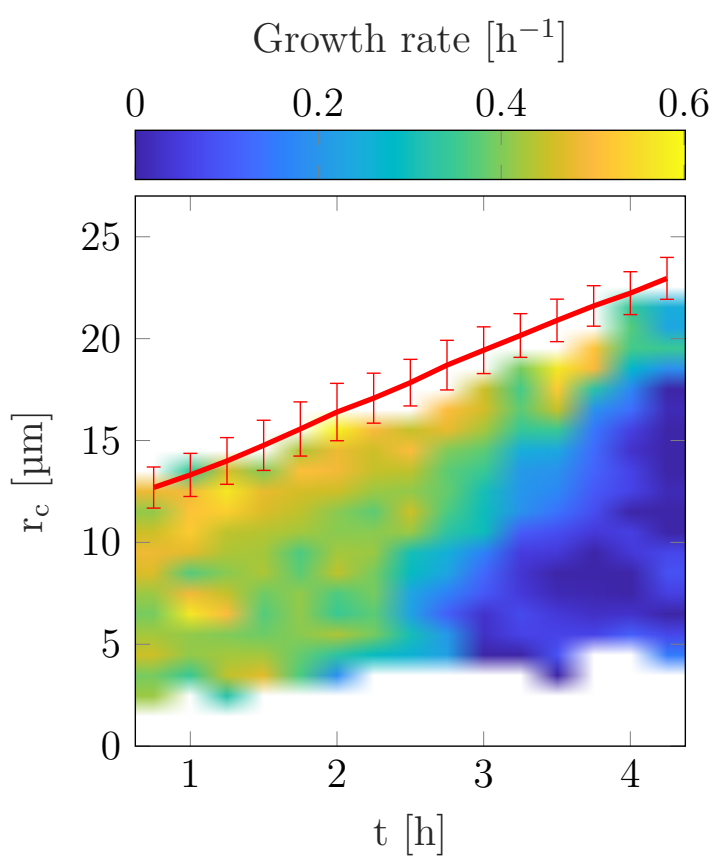

(a)

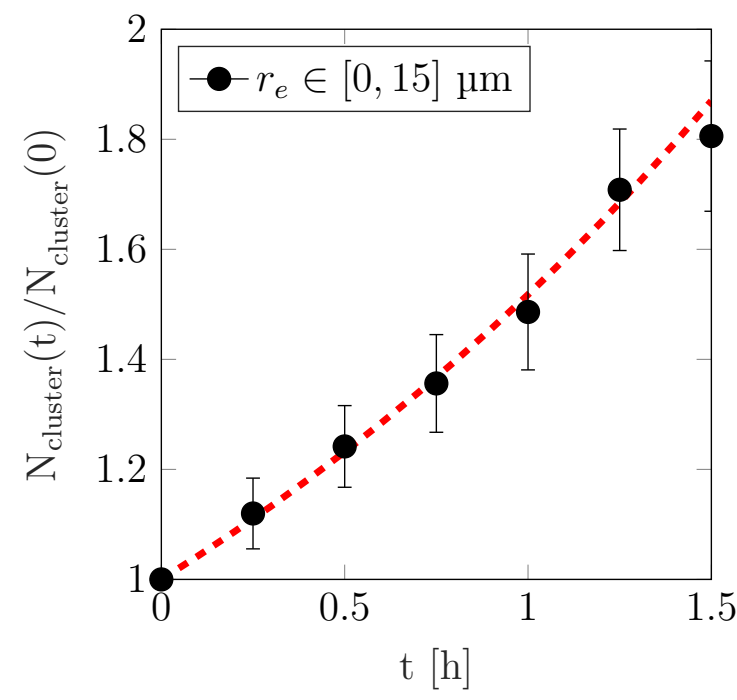

(c)

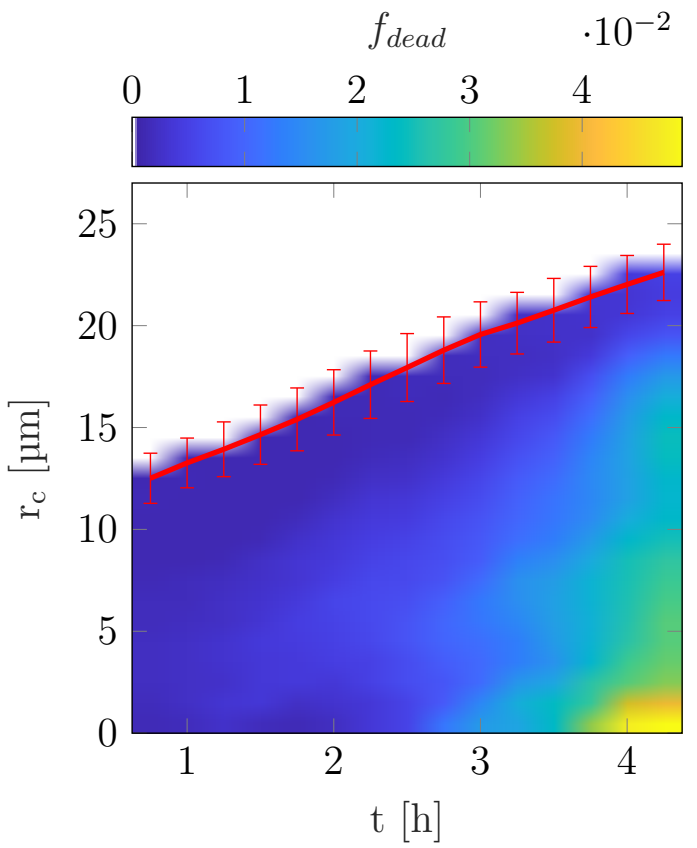

(b)

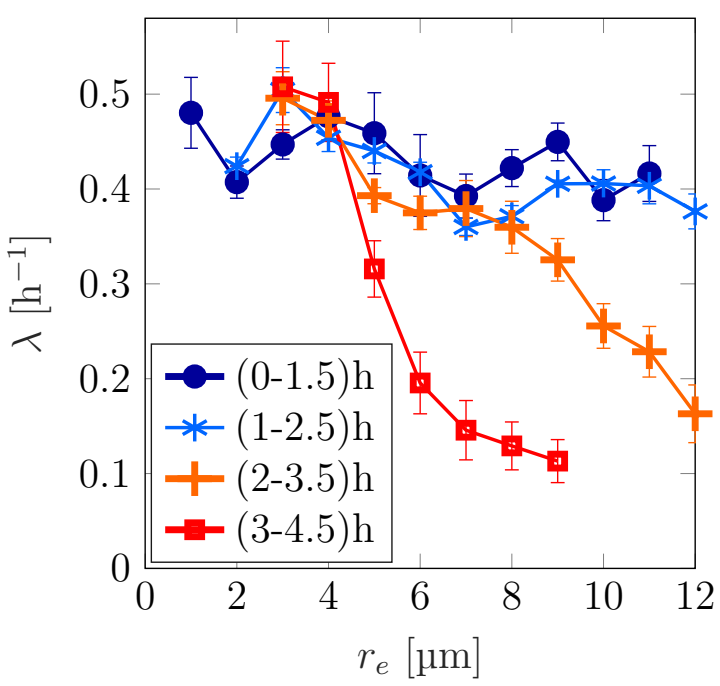

(d)

Figure 6.10: Spatio-temporal dynamics of growth of wt green ( $\mathrm{Ng} 194)$ mixed with $w t$ (Ng150) obtained by CC method. a) Growth rate $\lambda$ (colour coded) as a function of distance from centre of colony $\left(r_{c}\right)$ and time $t . \mathrm{b}$ ) Fraction of dead cells $f_{\text {dead }}$ (color coded) as a function of distance from centre of colony $\left(r_{c}\right)$ and time $t$. Red: Mean colony radius ( \pm standard deviation) as a function of time. c) Example of an exponential fit (red dotted) $\lambda_{\text {green }}=0.42 \pm 0.03$ to the averaged normalized number of cells $N(t+\tau) / N(t)$ inside clusters during the first $1.5 \mathrm{~h}$ and distance from the colony edge $r_{e}$ ( \pm se). Number of analysed clusters was 662 . d) Mean growth rate $\lambda$ as a function of the distance $r_{e}$ (mean \pm se, 10-285 clusters for each data point). 
of colonies and decreased towards the edges, where the growth rates were the highest. The calculation of the fraction of dead cells depends on density, which was assumed to be constant. Since the density decreases towards the colony edge at a distance of $3 \mu \mathrm{m}$ (Fig. $5.9 \mathrm{~b}$ ), the fraction of dead cells closer than $3 \mu \mathrm{m}$ was overestimated.

Using the cell counting method, we determined the effective growth rate, which is the difference between growth rate and death rate. Cells that were initially fluorescent could have died and lost their fluorescence. As a result, less cells per cluster would have been counted. However, the fraction of dead cells for each position and time point was smaller than $5 \%$ (Fig. 6.10b) and, therefore, we will neglect the death rate. In the following and our effective growth rate is similar to the growth rate.

It is unclear how growth rates of individual clusters evolve with time. To assess this question, we plotted the distribution of growth rates obtained from single clusters. During the first $1.5 \mathrm{~h}$, growth rates are distributed around a maximum of $\lambda_{\text {single }} \approx 0.5 h^{-1}$. This growth rate is higher than the growth rate determined by a single exponential fit to the average number of cells inside clusters obtained during the first $1.5 \mathrm{~h} \lambda_{\text {green }}=0.42 \pm 0.03$ (Fig. 6.10c). The difference in growth rates can be explained by the discrete peak at $\lambda_{\text {single }}=0 h^{-1}$ (Fig. 6.11a). Later the average growth rate of $\lambda_{\text {single }}$ shifts to lower values as a function of time (Fig. 6.11b,c,d) and the discrete peak at $\lambda_{\text {single }}=0 h^{-1}$ is persevered. Growth rates contributing to the discrete peak are calculated when the number of cells per cluster in the given time interval does not change. This is the case when cells do not divide, because their effective generation time is larger than $1.5 \mathrm{~h}$ or when the death rate is similar to the growth rate. The first scenario is likely to happen in the early biofilm stages where the number of cells per cluster is low (Fig. 6.11a,b). Additionally, cells that reside in a lag phase do not grow in the beginning. The second scenario is likely to happen in older biofilm stages where the fraction of dead cells increases (Fig. 6.11c,d). 

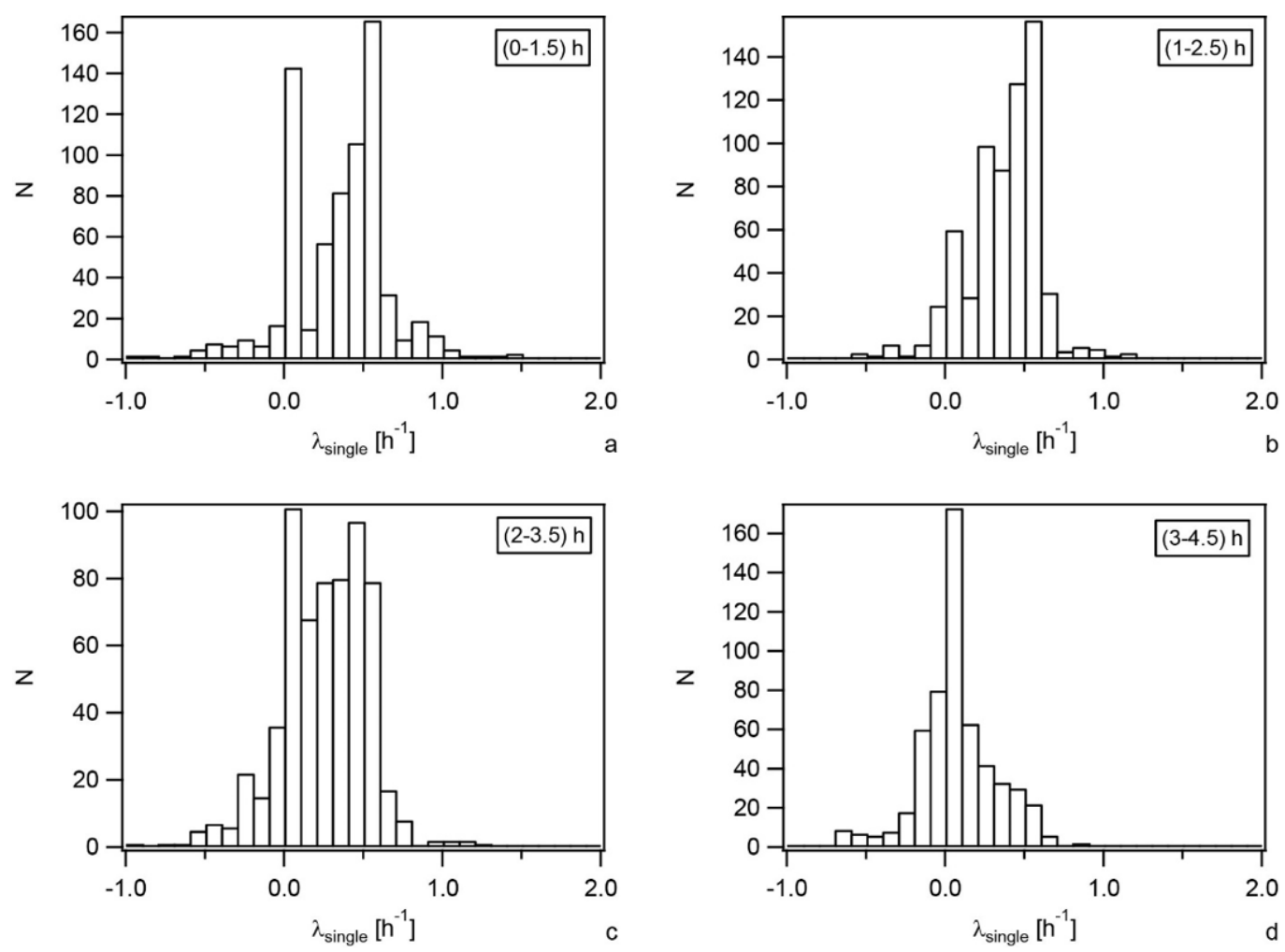

Figure 6.11: Effect of data averaging and fitting on determination of growth rates for wt green (Ng194) mixed withwt (Ng150). Histogram of growth rates obtained from single clusters by fitting over different time intervals of $1.5 \mathrm{~h}$ length as described in the methods section. Image adapted from $[172]$

\section{Growth profiles can be determined by the radial displacement with velocity field (VF) method}

Inside bacterial biofilms the space is limited. In order to grow and divide, cells have to push their neighbors towards the biofilm periphery (Fig. 6.12). Given a constant density, this effect generates a velocity field, where cells located close to the periphery of colonies show a higher velocity compared to cells that are further away (Fig. 6.13a). In the case of gonococcal colonies, a spherical symmetry is given. Thus, the velocity depends solely on the distance to the COM of colonies and can be used to determine the growth profile throughout colonies. To this end, 


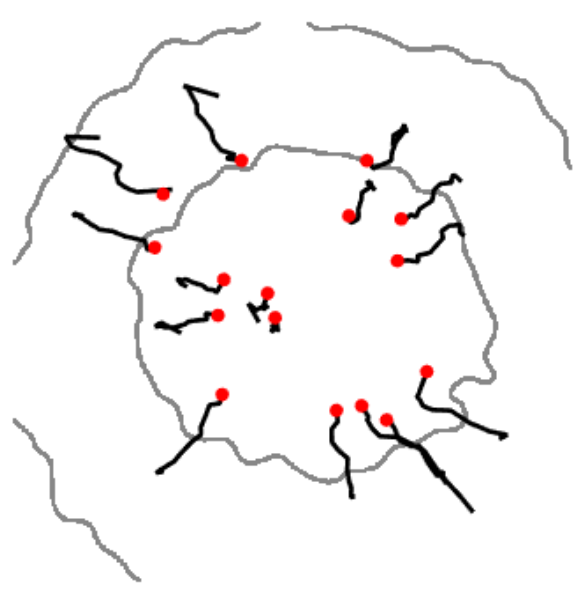

Figure 6.12: Trajectories of individual clusters moving within one colony acquired over $3 \mathrm{~h}$. Clusters residing at different heights within the colony are projected into one plane. Red circles: Start of trajectory, black lines: trajectories, grey lines: outlines of colony at $0 \mathrm{~h}$ and $3 \mathrm{~h}$, respectively. Image adapted from [172].

we calculated the radial velocities from our trajectories of clusters $v\left(r_{c}\right)=\vec{v} * \vec{e}_{r}$, which were projected on the unit vector $\vec{e}_{r}$ and pointed from the COM of colonies away. Velocities were determined by a linear fit $r_{c}(t)=v_{\text {radial }} * t+r_{c}(0)$ within 1.5 $\mathrm{h}$ time intervals.

For colonies younger than $2 \mathrm{~h}$, the velocity was close to zero at the colony COM and increased linearly as a function of $r_{c}$ with $v\left(r_{c}\right)=r_{c} *(0.17 \pm 0.01) h^{-1}$ (Fig. 6.13c). This confirms the initial homogeneous, exponential growth in the first two hours obtained by the colony radius method (Fig. 6.7). Later, the velocity field $v\left(r_{c}\right)$ became non-linear. In order to calculate growth rates from our velocity fields, we solved the continuity equation $\dot{\rho}=-\vec{\nabla} \cdot(\vec{v} \rho)+\lambda \rho$ numerically by assuming a constant cell density. We showed that the density was homogeneous in gonococcal colonies (Fig. 5.9b) except for distances closer than $3 \mu \mathrm{m}$ away from the edge of colonies. In this range the the density decreased and thus did not fulfill our assumptions to solve the continuity equation. Hence, distances closer than $3 \mu \mathrm{m}$ away from the edge of colonies were disregarded. 


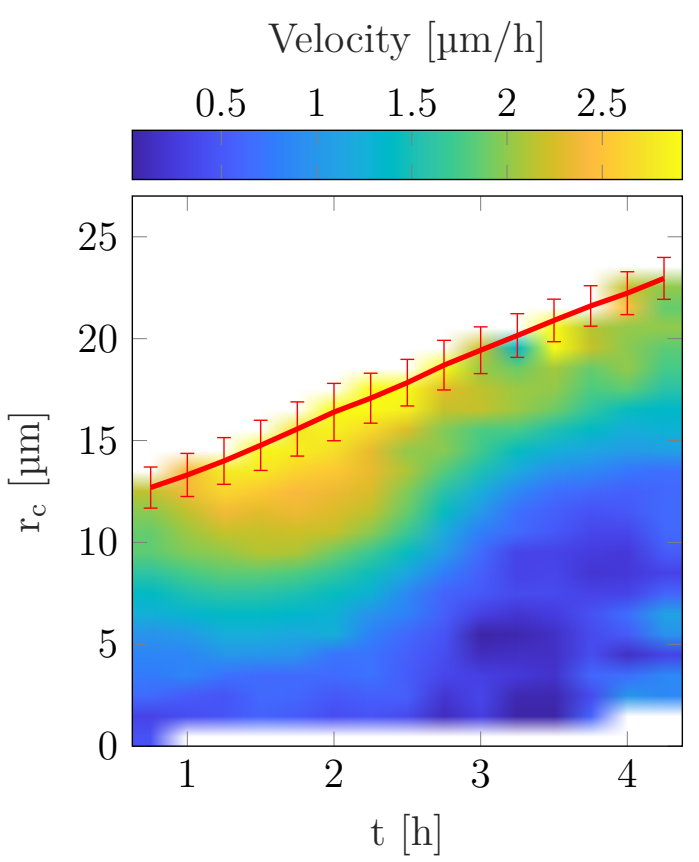

(a)

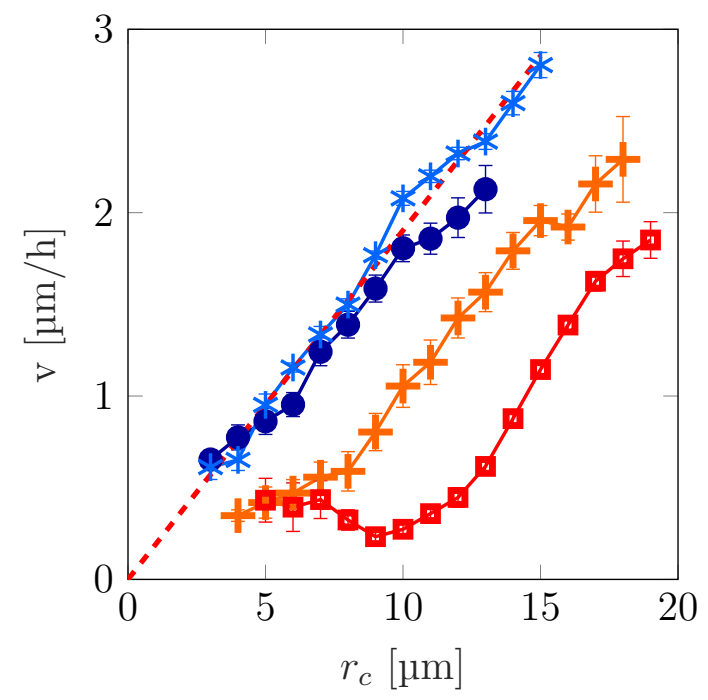

(c)

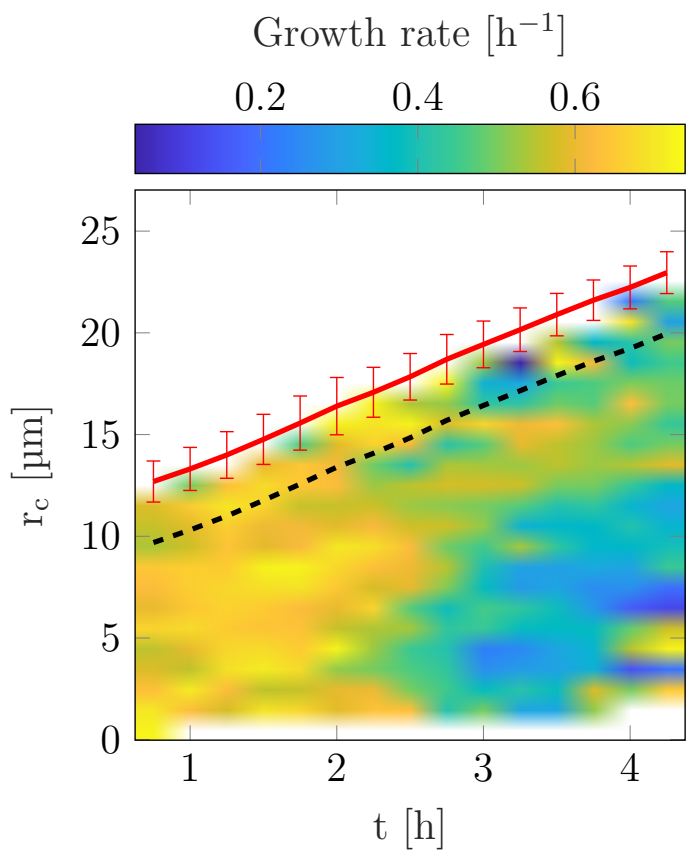

(b)

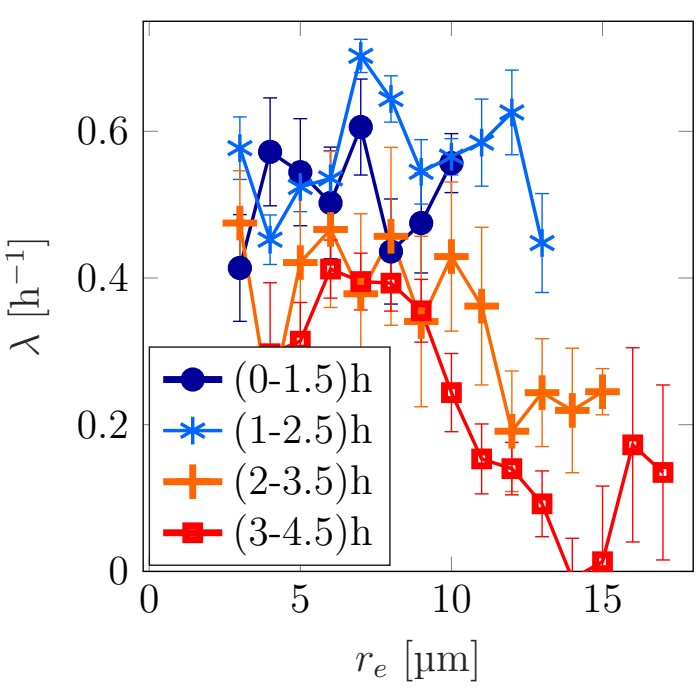

(d)

Figure 6.13: Growth rate determined from velocity field of clusters (VF method) of $w$ t green mixed with $w t$. a) Radial velocities $v$ of clusters as a function of distance to colony COM $\left(r_{c}\right)$ and time $t$. b) Growth rates $\lambda$ color coded) derived from velocity field. Red line: Mean colony radius ( \pm standard deviation), black dotted line: denotes area in which reduced cell density introduces a systematic error to $\lambda$. c) Velocity of clusters $v\left(r_{c}\right)$. Red dotted line: Linear fit to $v\left(r_{c}\right)$ for $(1-$ 2.5) h with $\lambda_{w t}=0.57 \pm 0.01$. d) Growth rates derived from velocity field. Error bars: mean \pm se, 10 - 212 clusters. 
Using spherical coordinates and assuming a constant density, the continuity equation simplifies to $\partial_{r}\left(r^{2} v_{r}\right)=r^{2} \lambda$. Growth rates were calculated consequently by $\lambda=\operatorname{div}(v)=2 \frac{v}{r_{c}}+\frac{d v}{d r_{c}}$. A linear velocity field simplifies this equation to $\lambda=3 * v_{\text {cluster }} / r$ and gives a growth rate of $\lambda_{w t}=0.57 \pm 0.01$ during the first $2 \mathrm{~h}$ (Fig. 6.13c). This value agrees well with the value obtained by the colony radius method (Chap. 6.2.1). For colonies older than 2 h, the simplified equation did not hold anymore due to a non-linear velocity field, consistent with a inhomogeneous growth profile for colonies older than $2 \mathrm{~h}$. Hence, growth profiles were determined by $\lambda(r)=2 \frac{v(r)}{r_{c}(r)}+\frac{v(r-1 \mu m)-v(r+1 \mu m)}{2 \mu m}$. The resulting growth profiles confirmed homogeneous growth in the first $2 \mathrm{~h}$ and decreasing growth rates towards the COM of colonies (Fig. 6.13b,d) consistent with the results from the CC method (Fig. $6.10 \mathrm{~d})$.

\subsubsection{Increasing nutrient supply does not prevent growth inhibition at the center of the colony}

To investigate the effect of nutrient supply on spatio-temporal growth and death dynamics, we improved the nutrient supply by increasing the flow rate of our peristaltic pump, which is connected to our flow chamber setup, 5 fold. Additionally, we limited the nutrient supply by diluting the growth medium. In general, our experiments were performed in GC medium, an undefined rich medium. For the experiment that addressed nutrient limitation, we supplemented PBS with $10 \%$ GC medium. All three conditions showed exponential growth (Fig. 6.14a) and homogeneous growth profiles (Fig. 6.14b) in the first $2 \mathrm{~h}$. After $2 \mathrm{~h}$, inhomogeneous growth profiles were observed (Fig. 6.14c,d). The improved nutrient supply due to a higher flow rate showed slightly higher growth rates, but did not prevent decreasing growth rates towards the $\mathrm{COM}$ of colonies. Hence, we assume that nutrient limitation was not the only reason for the inhomogeneous growth dynamics. Unexpectedly, growth rates close to the edge of colonies decreased after $2 \mathrm{~h}$ for the PBS diluted condition, causing a peak in the growth profile around 7 to $8 \mu \mathrm{m}$ away from the edge of colonies after $2.5 \mathrm{~h}$ (Fig. 6.14d) . The profile of fractions of dead cells showed similar behavior (Fig. 6.15b). 


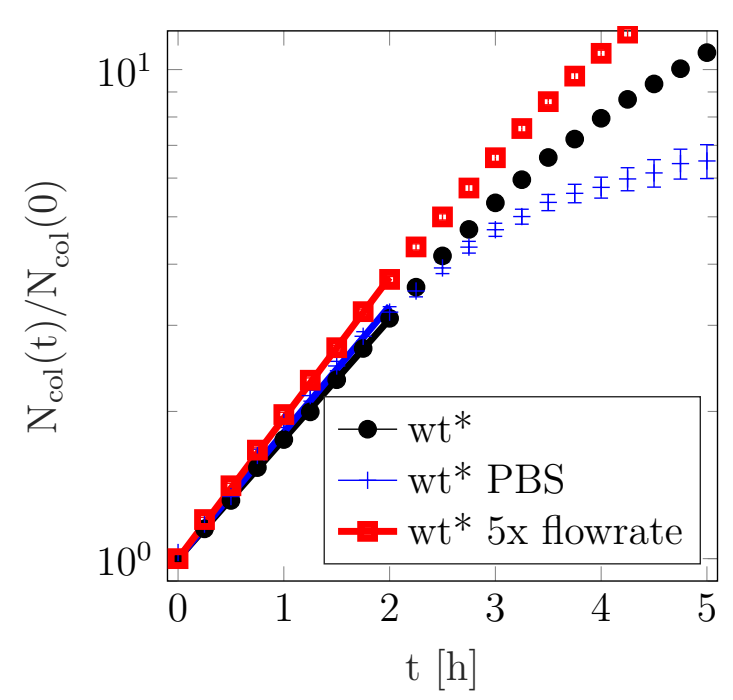

(a)

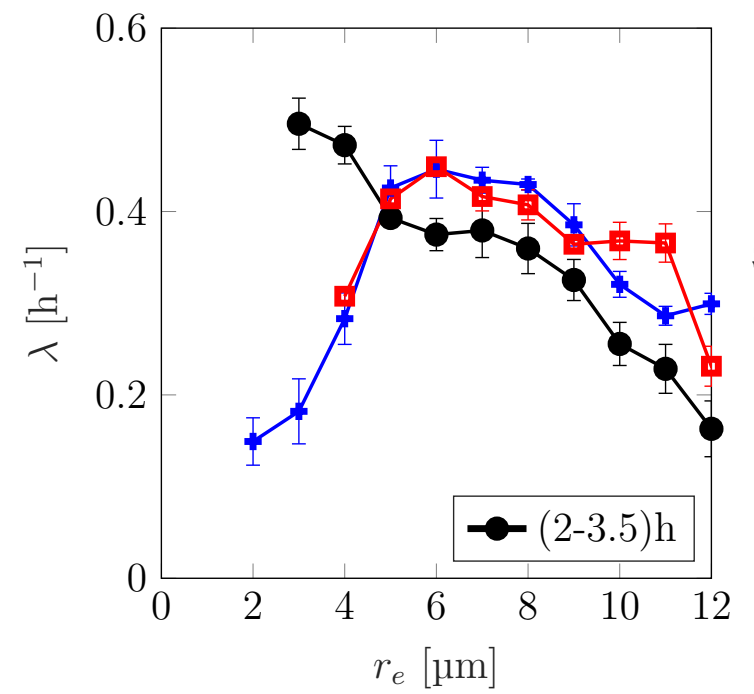

(c)

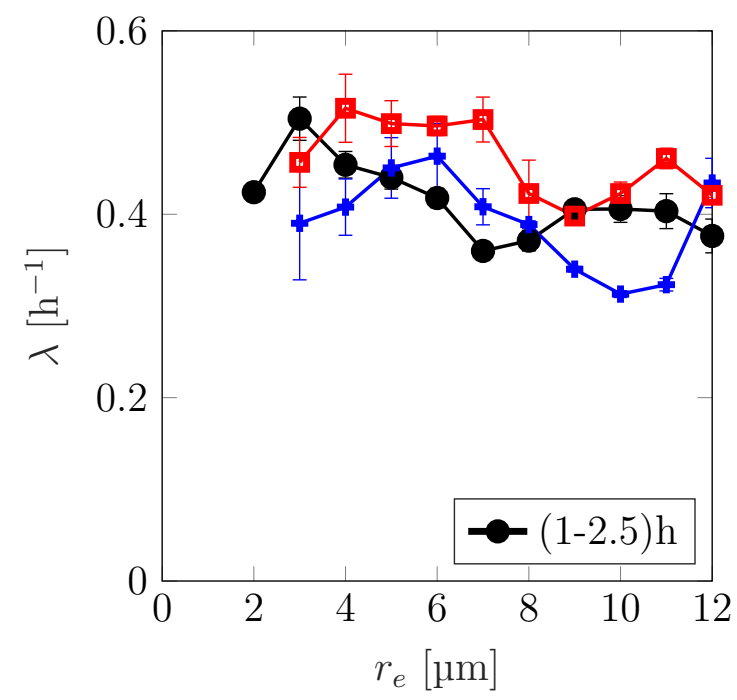

(b)

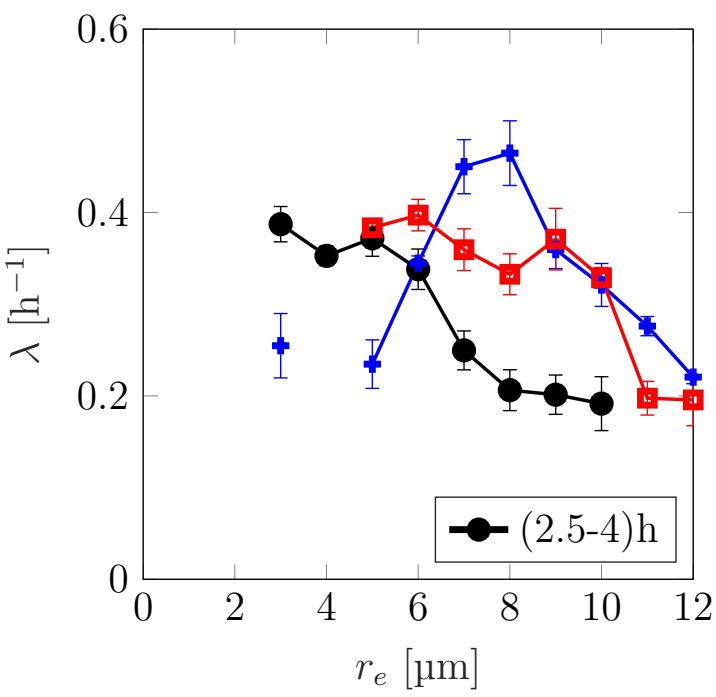

(d)

Figure 6.14: Effect of nutrients supply on growth rates. Comparison of growth profiles between $w t$ at standard conditions (black), 5 fold flow rate (red) and PBS diluted growth medium (blue). a) Averaged and normalized number of cells within colony $N_{\text {col }}(t) / N_{\text {col }}(0)$ (mean \pm se). Full lines: exponential fit with $N_{\text {col }}(t) / N_{c o l}(0)=\exp (\lambda t)$ gives $\lambda_{w t}=0.563 \pm 0.003, \lambda_{w t, P B S}=0.595 \pm 0.013$ and $\lambda_{w t, 5 x}=0.662 \pm 0.005$. b-d) Mean growth rates as a function of distance to the colony edge $r_{e}$ (mean $\pm \mathrm{se}$ ) derived by CC method. $\mathrm{N}>10$ clusters for each data point. 
Interestingly, nutrient limitation due to growth medium dilution played an important role in the distribution of the fraction of dead cells, causing an almost 7 times higher fraction of dead cells $12 \mu \mathrm{m}$ away from the colony edge after $2.5 \mathrm{~h}$ (Fig. 6.15b), while the improved nutrient supply caused a much smaller fraction of dead cells in the corresponding region. In summary, the nutrient supply plays an import role for spatio-temporal growth and death dynamics, but it is not solely responsible for our observed inhomogeneous growth profiles.

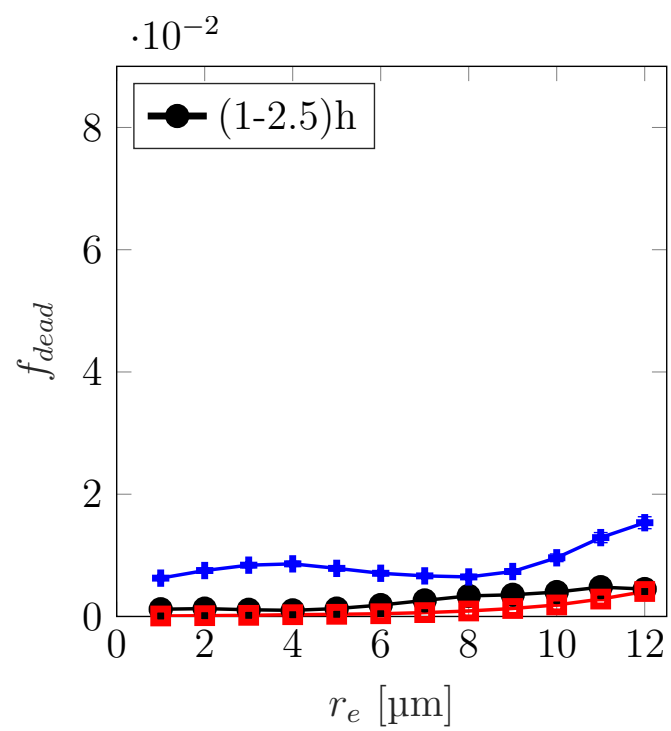

(a)

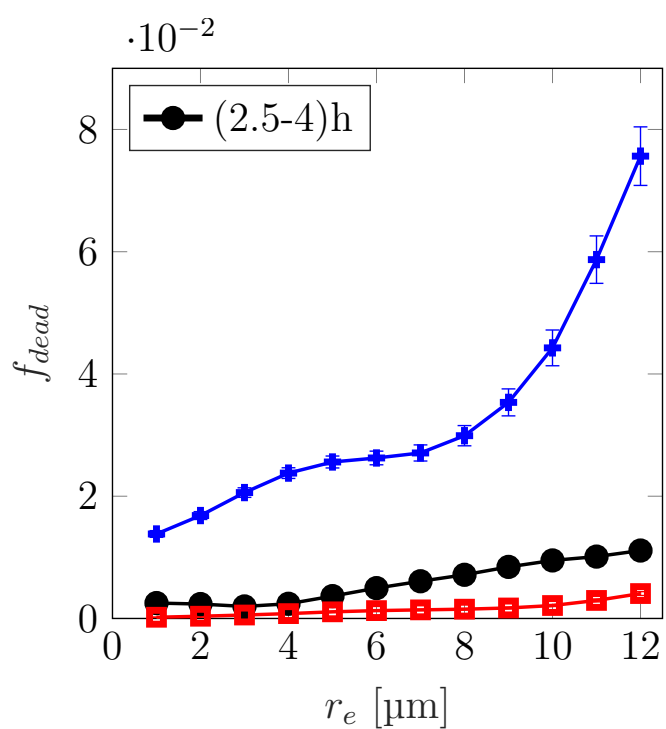

(b)

Figure 6.15: Effect of nutrients supply on bacterial death. Comparison of death profiles between $w t$ at standard conditions (black), 5 fold flow rate (red) and PBS diluted growth medium (blue). a-b) Averaged fraction of dead cells $f_{\text {dead }}$ (mean \pm se) within corresponding time interval as a function of distance to the colony edge $r_{e}$.

\subsubsection{Lack of stringent response accelerates heterogeneity in spatio-temporal growth dynamics}

In the previous section, we found that nutrient availability affects the spatiotemporal dynamics of growth and death. Here, we investigate the effect of a regulator which is activated as a result of nutrient limitation. We generated a strain lacking the $\Delta \operatorname{rel} A \Delta s p o T$ genes, which is not able to trigger stringent 


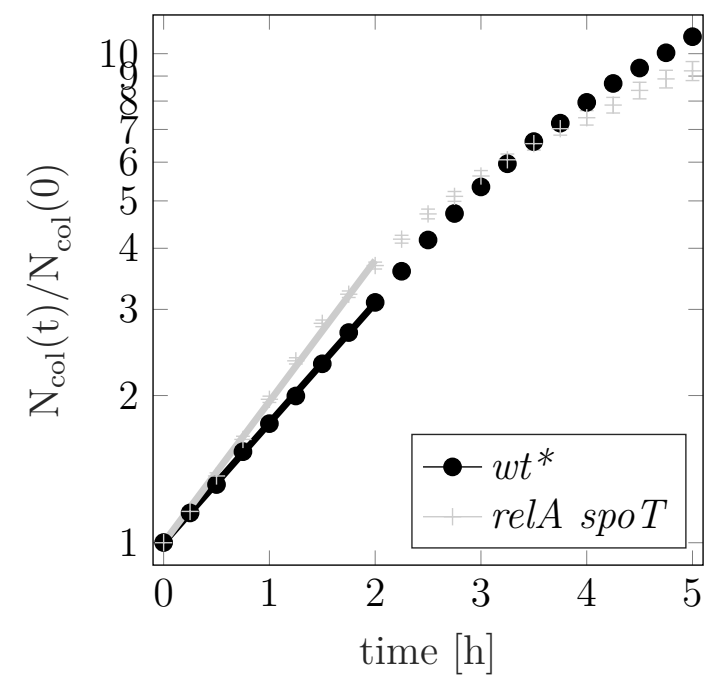

(a)

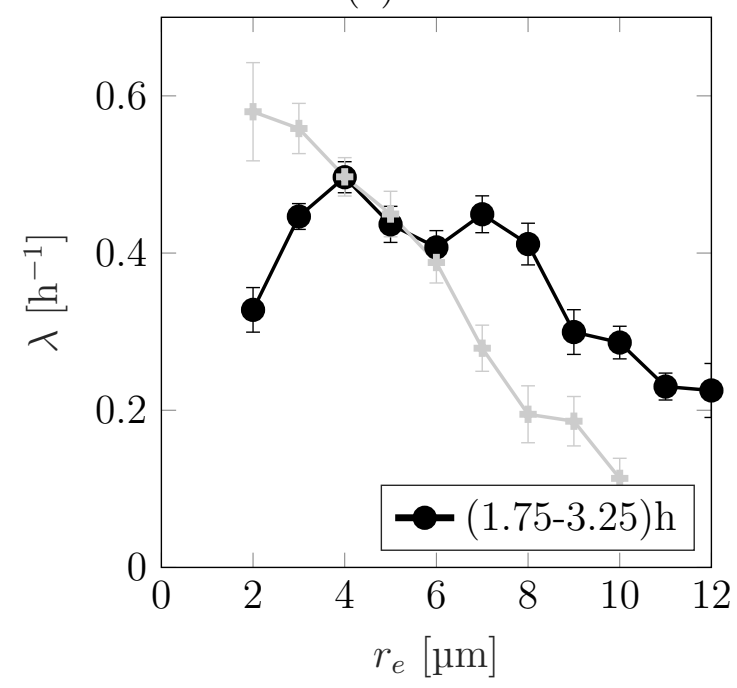

(c)

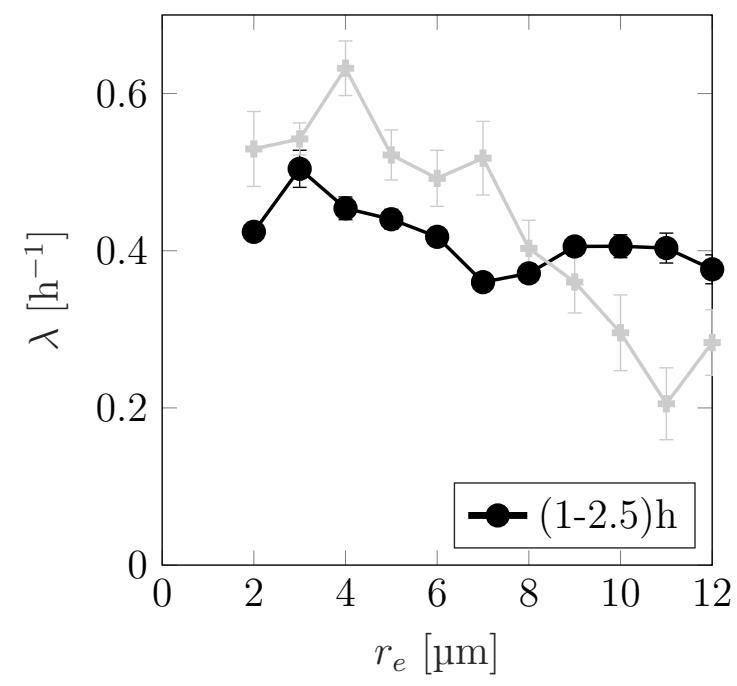

(b)

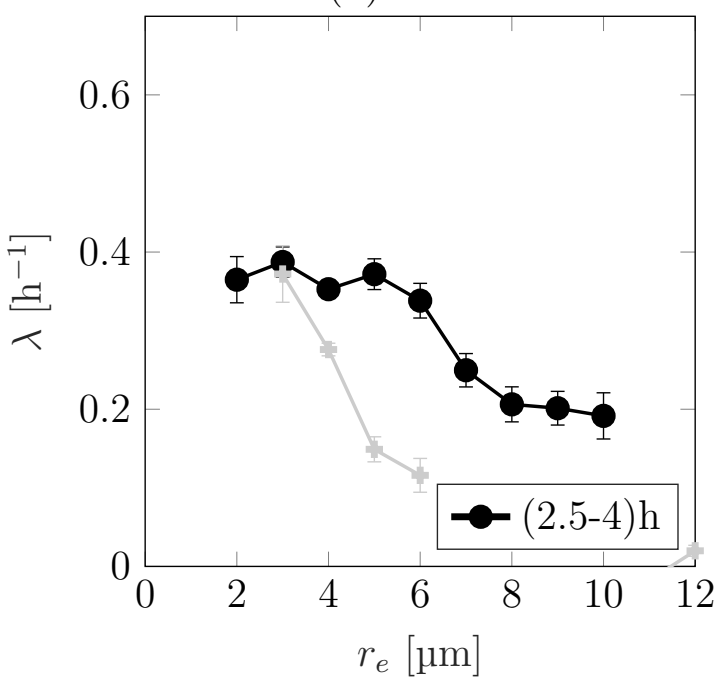

(d)

Figure 6.16: Effect of stringed response on growth rates. Comparison of growth profiles between $w t$ (black) and $\Delta \operatorname{rel} A \Delta s p o T$ (grey) strains. a) Averaged and normalized number of cells within colony $N_{c o l}(t) / N_{c o l}(0)$ (mean \pm se). Full lines: exponential fit with $N_{\text {col }}(t) / N_{\text {col }}(0)=\exp (\lambda t)$ gives $\lambda_{w t}=0.563 \pm 0.003$ and $\lambda_{\Delta r e l A \Delta s p o T}=0.66 \pm 0.01$. b-d) Mean growth rates as a function of distance to the colony edge $r_{e}$ (mean \pm se) derived by $\mathrm{CC}$ method. $\mathrm{N}>5$ clusters for each data point. 
response. The stringent response is triggered during nutrient starvation, when amino acids, carbon or fatty acids are limited [111, 112] due to accumulation of (p)ppGpp [113]. Deletion of the relA gene inhibits the production of (p)ppGpp and deletion of spoT compensates the growth defects caused by relA deletion alone [170]. To investigate the effect of stringent response on spatio-temporal growth and death dynamics, we mixed $\Delta$ relA $\Delta$ spoT and $\Delta$ relA $\Delta$ spoTgreenCells that where initially and let them grow in our flow chamber setup. Using the CC method, the effective growth rates were determined with spatial and temporal resolution. During the first $2 \mathrm{~h}, \Delta \operatorname{rel} A \Delta s p o T$ colonies grew faster than $w t$ colonies, but the grow rates decreased afterwards (Fig. 6.16a). Interestingly, heterogeneous growth profiles emerged in the $\Delta$ rel $A \Delta$ spoTgreen strain already after $1 \mathrm{~h}$, where the growth rates decreased towards the colony COM (Fig. 6.16b). By comparing growth rates at later time intervals, growth rates of $\Delta$ rel $A \Delta$ spoTgreen start to decrease closer to the edge of the colony than growth rates of wt green (Fig. $6.16 \mathrm{c}, \mathrm{d})$. Additionally, the fraction of dead cells $12 \mu \mathrm{m}$ from the edge of colonies

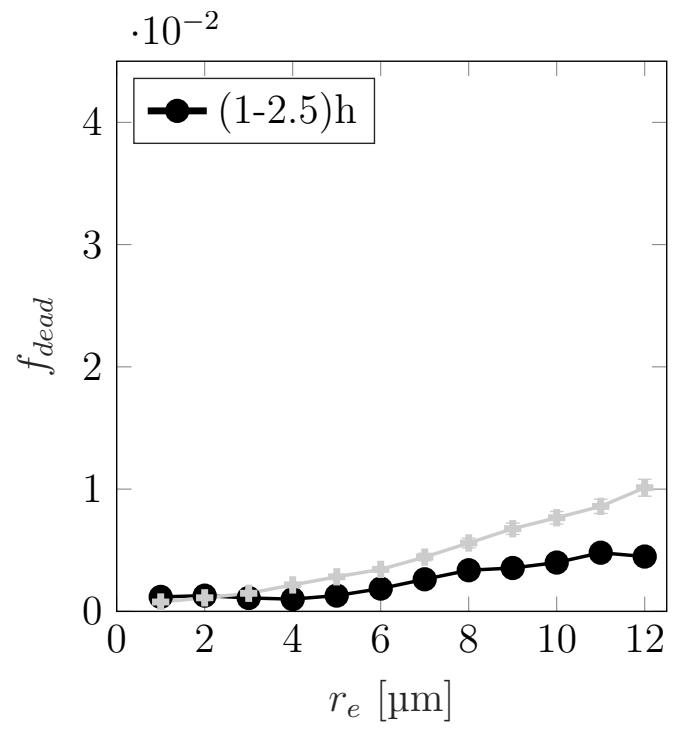

(a)

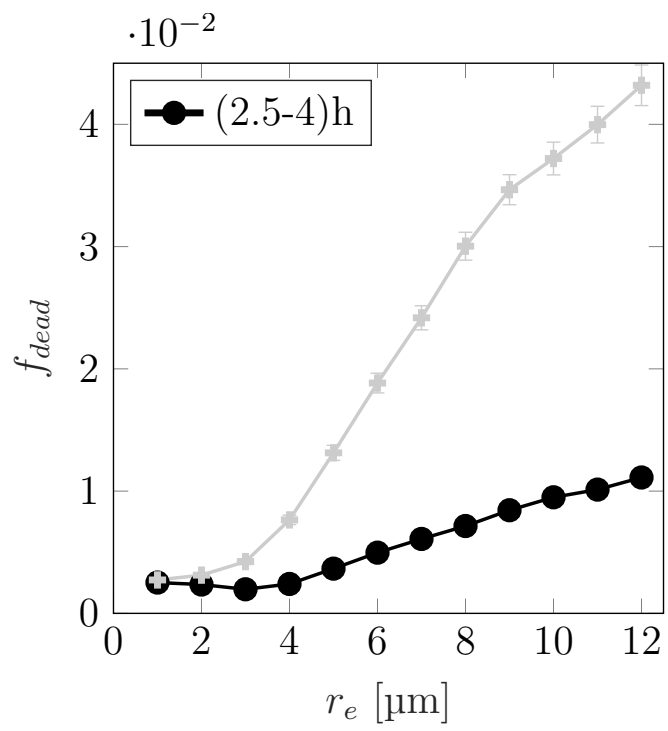

(b)

Figure 6.17: Effect of stringent response on bacterial death. Comparison of death profiles between $w t$ (black) and $\Delta \operatorname{rel} A \Delta s p o T$ (grey) strains. a-b) Averaged fraction of dead cells $f_{\text {dead }}$ (mean \pm se) within corresponding time interval as a function of distance to the colony edge $r_{e}$. 
was four times larger (Fig. 6.17), indicating that the stringent response plays an important role in the adaptation to environmental changes.

\subsubsection{Effect of antibiotic treatment on growth and death dynamics of gonococcal colonies}

We studied the effect of antibiotic treatment on growth and death dynamics of gonococcal colonies, which were constantly treated by supplementing the growth medium with the $2.5 \mathrm{x}$ MIC of azithromycin $(0.064 \mu \mathrm{mg} / \mathrm{ml})$ for $4.5 \mathrm{~h}$. Azithromycin prevents protein synthesis [188] and is currently recommended for treating infections caused by $N$. gonorrhoeae together with ceftriaxone [132]. Initially, growth rates of treated and non-treated colonies were very similar to each other (Fig. $6.18 a, b)$.

After $2 \mathrm{~h}$, we observed a negative effect of the azithromycin treatment on growth, where growth rates decreased close to the edge of colonies (Fig. 6.18c). Half an hour later, we could not detect sufficient sfGFP signal for cell localization at distances closer than $5 \mathrm{\mu m}$ from the colony edge (Fig. 6.18d), indicating a reduced expression of sfgfp. The fraction of dead cells $12 \mu \mathrm{m}$ away from the edge of colonies increased two-fold after 2.5 hours of azithromycin treatment (Fig. 6.19). In conclusion, we are able to measure the effect of azithromycin treatment on growth and death of individual cells inside colonies. The applied method can be also used to study the effect of other antimicrobial substances. 


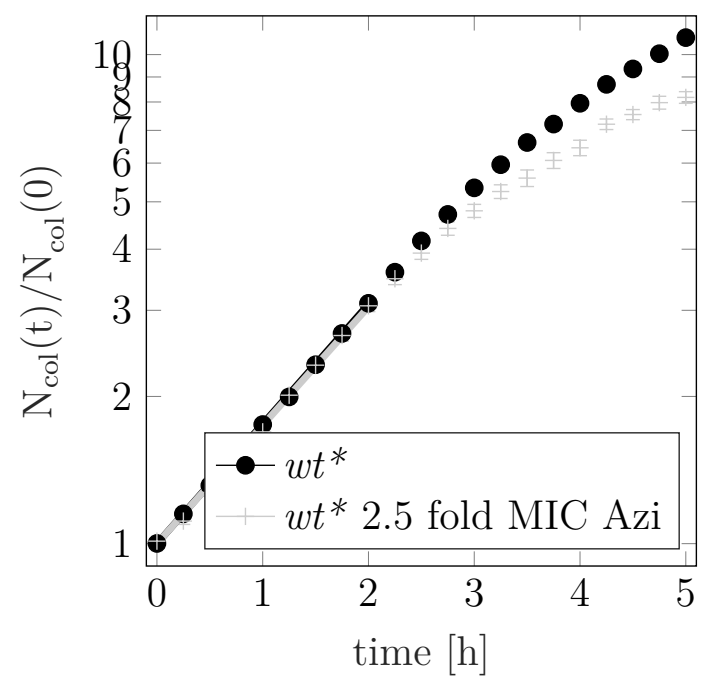

(a)

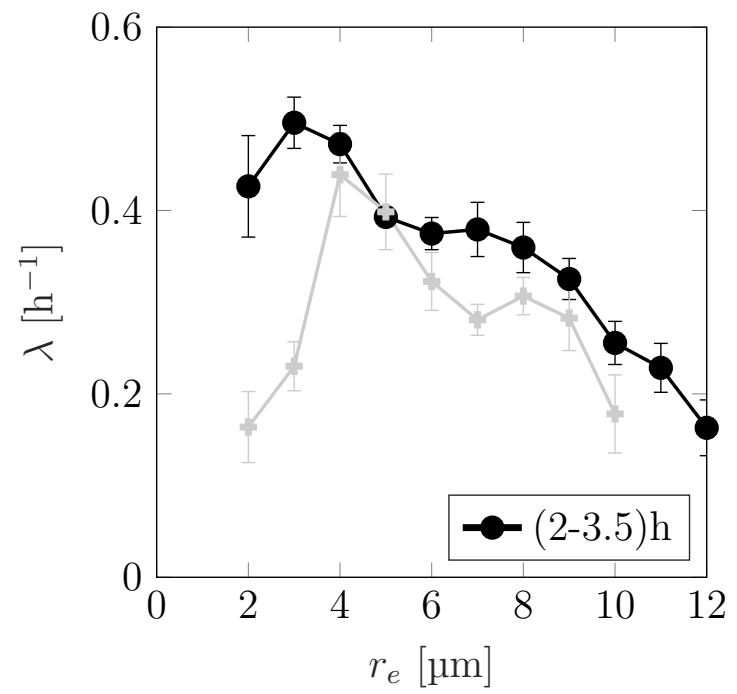

(c)

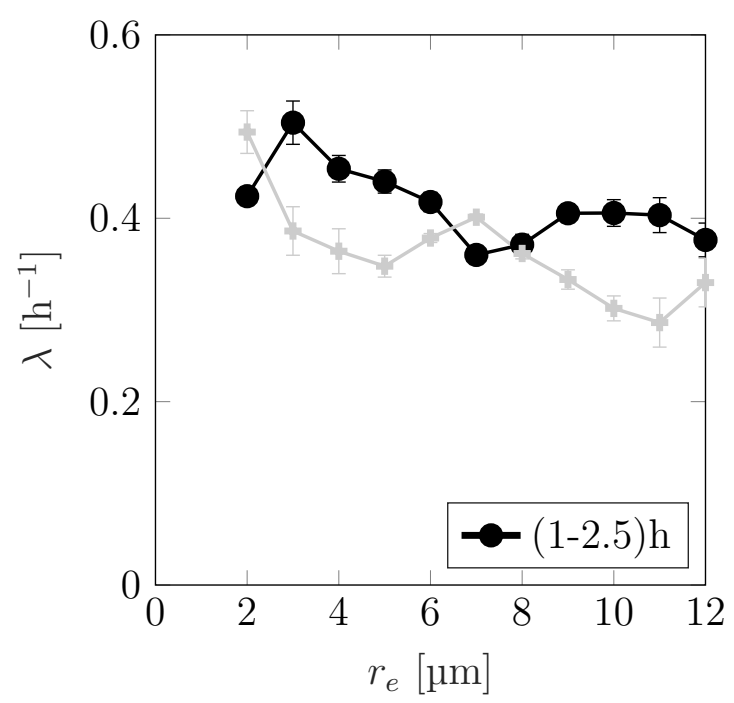

(b)

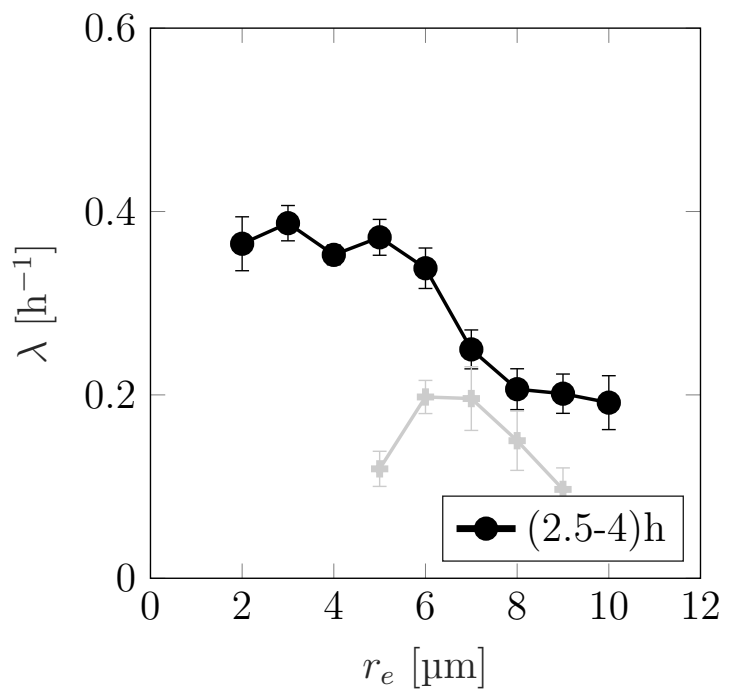

(d)

Figure 6.18: Effect of azithromycin treatment on growth rates. Comparison of growth profiles between $w t$ (black) and wt treated with 2.5 fold MIC of azithromycin (grey). a) Averaged and normalized number of cells within colony $N_{c o l}(t) / N_{c o l}(0)$ (mean \pm se). Full lines: exponential fit with $N_{\text {col }}(t) / N_{\text {col }}(0)=\exp (\lambda t)$ gives $\lambda_{w t}=0.563 \pm 0.003$ and $\lambda_{w t a z i}=0.55 \pm 0.01$. b-d) Mean growth rates as a function of distance to the colony edge $r_{e}$ (mean \pm se) derived by $\mathrm{CC}$ method. $\mathrm{N}>5$ clusters for each data point. 


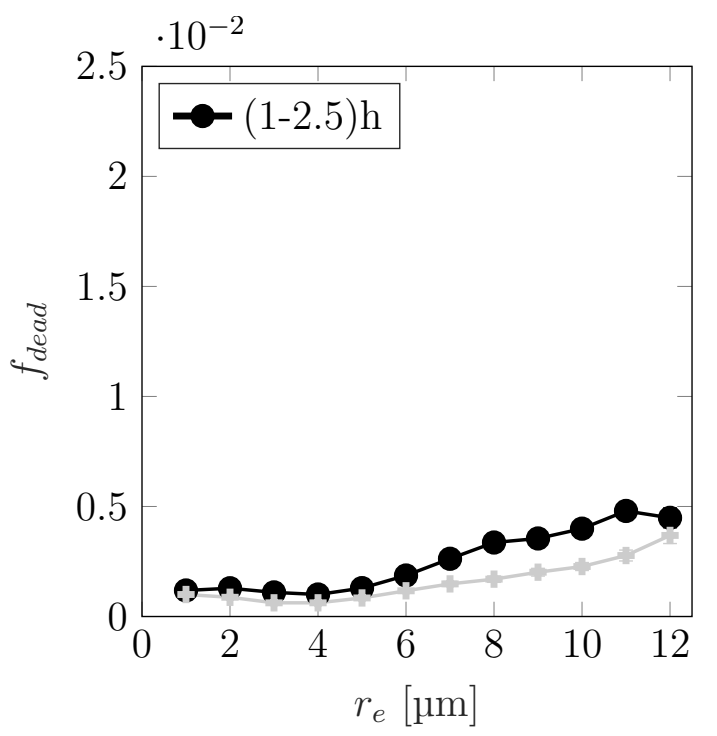

(a)

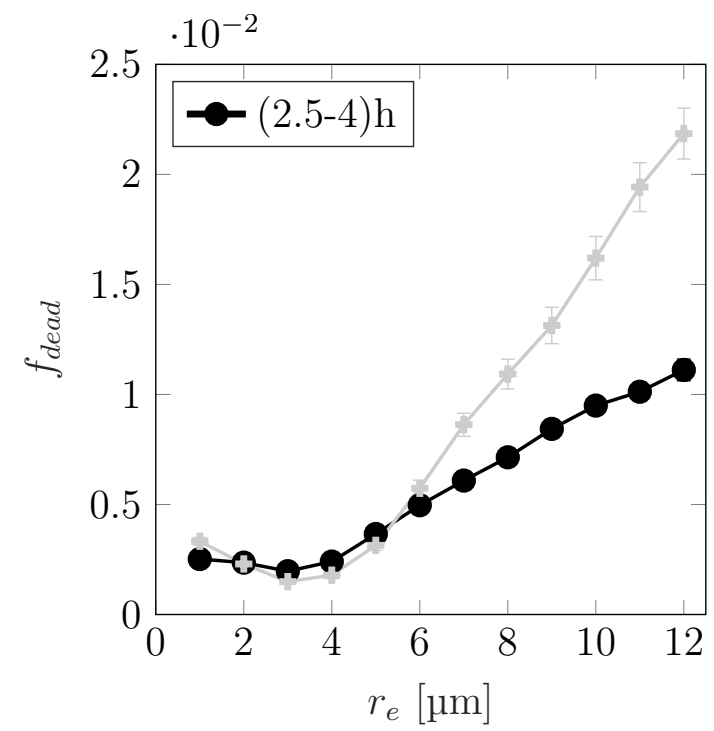

(b)

Figure 6.19: Effect of azithromycin treatment on bacterial death. Comparison of death profiles between $w t$ (black) and $w t$ treated with 2.5 fold MIC of azithromycin (grey). a-b) Averaged fraction of dead cells $f_{\text {dead }}$ (mean $\pm \mathrm{se}$ ) within corresponding time interval as a function of distance to the colony edge $r_{e}$. 


\section{Discussion}

\subsection{Gonococcal colonies are reminiscent of non-living colloidal systems}

Gonococcal cells are similar to colloidal systems in some aspects. They can be considered as hard spheres that sterically repulse each other. T4P generate attractive force between neighboring cells. These properties are reminiscent of 1 $\mu \mathrm{m}$-sized latex beads which are functionalized in a way that they attract each other. Attractive interactions for colloidal particles can be mediated for example by decorating colloids with DNA [189] or proteins [190]. When the interaction between colloidal particles is mediated through DNA hybridization, diffusionlimited self-assembly of aggregates can be triggered by temperature change. After approximately $50 \mathrm{~h}$, these colloidal aggregates show crystal-like structures [189]. Alternatively, self-assembly can be triggered by light stimulation. When colloidal particles are functionalized with photoswitchable proteins like VVDHigh or Cph1, blue or red light emission triggers self-assembly. When these aggregates are placed in the dark, they start to disperse [189].

In contrast to colloidal particles that form small aggregates with crystalline order [189], gonococci form large aggregates with short-ranged order similar to liquids. Aggregates of colloidal particles are typically assembled by identical particles, but gonococcal colonies consist of spherical and dumbbell-shaped cells. Initially, cells are spherically shaped and grow into dumbbells prior cell division. The mixture of spherical and dumbbell-shaped cells causes polydispersity and a symmetry break that is likely to inhibit the formation of long-ranged order. However, in-silicio colonies that consist solely of dumbbells do not show polydispersity, but their 
structure shows also a short-ranged order [191]. While the assembly of colloidal aggregates is typically diffusion limited [189], gonococci form their colonies actively via T4P interactions. Consequently, it is likely that the dynamic rearrangement caused by T4P dynamics and growth of bacteria within the colony inhibits the formation of crystalline order.

\subsection{Deletion of the T4P retraction motor severely slows down the process of local ordering}

Gonococci form spherically shaped colonies, which show similarities to water droplets. Two micro-colonies in close proximity minimize their surface area by fusing to a larger spherical colony in the order of minutes $[44,50]$. The fusion dynamics suggests that gonococcal micro-colonies can be described as a liquid phase with an effective surface tension. In computer simulations their architecture [191, 192] and temporal dynamics [75] were confirmed to be liquid-like. In order to study the importance of the T4P retraction motor PilT experimentally, strains with different T4P motor properties were characterized by investigating shape relaxations of two coalescing micro-colonies [44]. Subsequently, the shape relaxation data was used to estimate the viscosity of micro-colonies. Micro-colonies formed by the $w t$ and pilT $_{W B 1}$ (Table 5.2) showed a viscosity of $\eta_{w t} \approx 350 \mathrm{Nsm}^{-2}$ and $\eta_{w t} \approx 2000 \mathrm{Nsm}^{-2}$. Formation of local order requires dynamic rearrangement of bacteria within the colony. Liquid-like colony dynamics enable these rearrangements.

Surprisingly, colonies formed by pil $_{W B 2}$ show a higher order and a lower cell density than $w t$ colonies at $6 \mathrm{~h}$. This effect could be explained by the higher pilus motor activity and the lower viscosity of $w t$ colonies that facilitates cell reorganization inside colonies allowing higher cell densities but causes less local order. Computer simulations investigated the effect of T4P-T4P interaction on the structure of colonies. They show that lower pilus retraction velocities increase the local order of in-silicio colonies [192]. Indeed, lower retraction velocities were measured for the pilT $T_{W B 2}$ mutant strain [44] highlighting the pilus retraction velocity as an important parameter that determines local order inside fluid-like colonies. 
Deletion of pilT induces a transition of fluid-like to solid-like behavior of the colony. A strain in which the gene encoding for the T4P retraction ATPase pilT is deleted, assembles more pili [193]. The RDF of the $\Delta$ pilT strain deviated from a fluid-like structure by showing no local order for $6 \mathrm{~h}$ old gonococcal colonies. Interestingly, local order emerged after $24 \mathrm{~h}$. We suggest that repulsive interaction due to bacterial growth causes gonococcal re-organization on a much longer time scale compared to active T4P force generation. Another reason for slow ordering could be T4P retraction in the absence of PilT. Surprisingly, pilus retraction was observed in the absence of PilT [194], but important functions like twitching motility and colony fluidization were not supported due to the strongly reduced pilus force generation. Despite slower and weaker T4P dynamics of the $\Delta$ pilT strain, pilus retraction possibly contributes to the bacterial ordering on longer time scales. For the closely related pathogen $N$. meningitidis, deletion of pilT caused the same effect. Meningococcal $\Delta$ pilT colonies were not spherical and did not flow into the pipette during the micropipette aspiration assay, confirming the lack of fluid-like properties [50]. In conclusion, T4P motor activity accelerates local ordering and PilT is crucial for fluid-like properties of bacterial colonies.

\subsection{Determining growth rates with spatial and temporal resolution in spherical colonies}

Typically, growth rates are determined by measuring the optical density (OD600) to determine bacterial concentration in a solution. Subsequently, an exponential function can be fitted to the estimated number of cells inside the solution. The main problem with this approach is that aggregation and surface attachment of cells influence the optical density, leading to inaccurate results [195]. To avoid this problem, the number of cells inside colonies can be estimated by measuring the colony radius. However, this approach gives solely the mean growth rate. In order to study growth rates with spatial and temporal resolution in spherical colonies, we developed two novel methods that have advantages and disadvantage.

Conceptually most straight forward but technically challenging way to char- 
acterize growth rates would be to start with a single founder cell and track its offspring. This method was successfully implemented for the rod-shaped bacterium $V$. cholerae [12], but cannot be applied for N. gonorrhoeae. For V. cholerae growth rates were determined by measuring the length of cells that increases prior cell division. Our cell localization approach does not access shape deformations or volume increase of cells. Consequently, we cannot obtain growth states of single cells or estimate orientations of dumbbells. In order to track the offspring of single cells, we need to apply particle tracking algorithms. These algorithms are not able to compute all possible combinations of trajectories, because the cell density inside gonococcal colonies is too high. Gonococci show a volume fraction of approximately 0.5 in their colonies, which is higher than the estimated volume fraction of 0.2 within $V$. cholerae colonies. Using spinning disk confocal microscopy, we were able to localize cells up to a height of $8 \mu \mathrm{m}$, when all cells were fluorescent. For higher image planes the SNR was drastically reduced, inhibiting localization of single cells. Hence, we mixed fluorescent and non-fluorescent cells to increase the SNR for higher image planes. This allowed localization and tracking of cell clusters at heights of $25 \mathrm{\mu m}$ relative to the bottom. The number of cells per cluster (CC method) and their displacement (VF method) were used to calculate growth rates. The main disadvantage of mixing fluorescent and non-fluorescent cells was that we needed to start experiments with a high concentration of cells in the flow chamber. We assume that the high concentration of cells caused deficiencies in nutrient availability after $2 \mathrm{~h}$. At that time, we observed development of heterogeneous growth rate distributions. In order to determine positions of single cells, we needed a strong fluorescent signal. Using the strong pilE promoter to express sfgfp, we observed a $25 \%$ drop in growth rate of compared to the wt strain. Thus, growth rates of the non-fluorescent $w t$ strain can be determined only indirectly from the wt green strain that shows a reduced growth rate.

In contrast to the $\mathrm{CC}$ method, growth rates derived using the VF method are based on the velocity field generated by non-fluorescent $w t$ strain. As a result, growth rates determined by the VF methods are dominated by wt cells and wt green cells serve as fluorescent markers for characterizing the velocity field. Between 1 and $2.5 \mathrm{~h}$, the CC method gives a spatial mean growth rate of $\lambda_{\text {wtgreen }}=(0.42 \pm 0.03) h^{-1}$ 
and the VF method $\lambda_{w t}=(0.57 \pm 0.01) h^{-1}$. From the colony radii, growth rates $\lambda_{\text {wgreen }}^{*}=(0.42 \pm 0.003) h^{-1}$ and $\lambda_{w t}^{*}=(0.563 \pm 0.003) h^{-1}$ were estimated, fitting very well to the growth rates determined by the CC and VF method. The application of the VF method method is limited to colonies with a constant density and cell packaging or cell size. We observed a decreasing density towards the gonococcal periphery of colonies, where the VF method cannot be applied. In the case of azithromycin treatment of gonococcal colonies, we observed cell swelling, which would influence the velocity field, leading to calculation of higher growth rates [5]. Additionally, the VF method depends strongly on the correct position of the COM of colonies. Especially, the COM height is hard to estimate, because it changes within time, limiting the accuracy of this method. In conclusion, the $\mathrm{CC}$ and the VF method have both their own advantages and disadvantages. By combining these two complementary approaches, they characterize growth profiles in gonococcal colonies robustly.

\subsection{Growth rate gradients emerge within a few generations and stringent response retards the development of spatially heterogeneous growth rates}

When $N$. gonorrhoeae is incubated in flow chambers as described in the methods (Chap. 5.3.2), the time that cells reside in spherical colonies is approximately $8 \mathrm{~h}$. During the first $2 \mathrm{~h}$, growth rates were spatially homogenous. Between 2 and $5 \mathrm{~h}$ growth rates became spatially heterogeneous. Within $3 \mu \mathrm{m}$ away from the surface of colonies, growth rates were constant. Interestingly, we measured in this area a reduced cell density. For larger distances from the colony edge, growth rates decreased proportional to colony age. Later, the fraction of dead cells increased and colony breakdown occurred. In the following discussion, we will focus us on the intermediate time range, where spatially heterogeneous growth rates were observed and cell death can be neglected. 
7.4 Growth rate gradients emerge within a few generations and stringent response retards the development of spatially heterogeneous growth rates

In order to test the effect of nutrient supply on the development of growth rate gradients, we increased the flow rate. Even though the decreasing growth rates towards the colony core were not prevented, the rapid drop of growth rates occurred at a larger distance around $10 \mu \mathrm{m}$ from the surface of colonies (Fig. $6.14 \mathrm{~d}$ ). In comparison, the control showed its drop at a similar time between 2.5 and $4 \mathrm{~h}$ around $6 \mu \mathrm{m}$. We conclude that a higher flow rate increases the nutrient availability for deeper layers, but due to the fact that cells closer to the periphery of colonies consume nutrients, the diffusion of nutrients decreases for larger distances from the surface of colonies. In a different approach, we diluted the nutrient concentration. Surprisingly, we observed that growth rates decreased at the surface of colonies and cell death became relevant. Growth rates between 2.5 and $4 \mathrm{~h}$ showed a peak around 7-8 $\mathrm{\mu m}$ away from the surface of colonies (Fig. 6.14 d). We assume that dead cells or cells with less active T4P are sorted towards the periphery of colonies. The strongly increased fraction of dead cells for larger distances from the surface of colonies indicate that nutrient availability is important for cell survival. Besides nutrient availability, spatially heterogeneous growth rates can be caused by mechanical constraints [196, 197, 198]. During colony maturation, an extracellular matrix is generated that probably increases the pressure inside colonies and affects growth rates negatively.

The stringent response is activated during limitations of various nutrients. Our first assumption was that the absence of the stringent response would decelerate development of heterogeneity, but we observed that growth rate gradients occurred earlier in $\Delta$ rel $A \Delta s p o T$ mutant colonies compared to wt colonies. The $\Delta$ rel $A \Delta s p o T$ strain does not activate the stringent response and showed higher growth rates during the first $2 \mathrm{~h}$ of colony growth. This growth rate difference between the $\Delta \operatorname{rel} A \Delta$ spoT strain and the control indicates that the stringent response is activated prior the development of heterogeneity. Consequently, the stringent response would be an early alert system that tries to maintain homogeneous growth and the absence of stringent response would cause emergence of growth rate gradients at an earlier time. In conclusion, the stringent response is an important feedback mechanism that optimizes the distribution of available 
nutrients and is important for adaptation to changing local environments during colony maturation [116].

\subsection{Azithromycin is more efficient against fast growing cells}

Typically, minimal inhibitory concentrations (MIC) are determined to test bacterial susceptibility to specific antibiotics. Even though the MIC measurements are considered to be the 'gold standard' [199], bacterial biofilms show a higher tolerance against antimicrobial treatment than their planktonic counterpart [118]. Since the majority of clinical infections are based on biofilms, the minimum biofilm eradication concentration was established, which defines the minimal concentration of a drug required to kill a biofilm by removing biofilms from the substrate and observe the number of living cells on agar plates [200]. Neither of these methods accesses the time needed until the antimicrobial treatment effects the cell growth.

In this study, we determined spatio-temporal growth rates inside gonococcal colonies with and without azithromycin treatment. Before cell death became relevant, reduced growth rates were observed, when gonococcal colonies were treated with the $2.5 \mathrm{MIC}$ of azithromycin. We currently do not know whether growth slows down because the concentration of proteins is affected or because a stress response has been launched. After approximately $3 \mathrm{~h}$ of colony growth, we could not localize fluorescent cell at distances closer than $5 \mu \mathrm{m}$ to the colony edge. Interestingly, growth rates were maximal in this range in our control. Fluorescent cells with a larger distance from the colony edge were successfully tracked and used to estimate growth rates. These growth rates were slightly smaller in comparison to the control. By treating sfGFP as a marker for protein synthesis, we can conclude that fast growing cells close to the colony surface are affected the most from the azithromycin treatment and that they express less proteins after $3 \mathrm{~h}$ of treatment. This hypothesis is confirmed by experiments that show higher antimicrobial tolerances for cells with a reduced metabolic activity $[92,54,93,94,95]$. 
Currently, gonorrhea is treated with a combination of azithromycin and ceftriaxone [132]. Interestingly, the tolerance of gonococcal colonies to the combination of these drugs strongly depends on the time offset, when each drug is applied. Cronenberg et al. showed that a sequential treatment with a time offset of $3 \mathrm{~h}$ increases the efficiency of the therapy in comparison to a simultaneous treatment [5]. Thus, the timing of sequential antimicrobial treatments besides absolute concentration values plays an important role. Assuming that ceftriaxone treatment is more efficient against cells with a high metabolic activity, the sequential ceftriaxone treatment should be initiated when growth rates of gonococcal colonies treated with azithromycin are high. We conclude that sequential treatment of azithromycin and ceftriaxone with a time offset slightly less than $3 \mathrm{~h}$ would be probably more efficient, because cells are more metabolically active. 


\section{Outlook}

In this study, we determined spatio-temporal growth rates and the distribution of dead cells inside gonococcal colonies. To this end, we used two different approaches, each one having its own advantages and disadvantages. Sfgfp fluorescent bacterial clusters were tracked inside non-fluorescent cell dominated colonies and velocity fields of these clusters were determined. The spatio-temporal velocity fields were used to calculate gonococcal growth rates. Especially, fluorescent cells show reduced growth rates and are more sensitive to laser light. To solve this problem, we could try to track dead cells. To improve the signal to noise ratio, we could use SYTOX staining instead of PI for staining cells. Additionally, we could probably increase the time resolution without effecting the growth of non-fluorescent cells, because they seem to be less sensitive to laser light. If dead cells follow simply the velocity field, which is generated by growing cells, spatio-temporal velocities could be used directly to calculate the corresponding growth rates with the VF method. Apart from simply following the velocity field, it seems quite likely that dead cells would be sorted to the periphery of colonies due to a reduced neighbor interaction. Thus, the comparison of spatio-temporal velocity fields obtained by living fluorescent cell clusters with the velocity fields of dead cells would give evidence whether cell sorting of living and dead cells occurs inside gonococcal colonies.

We observed the emergence of heterogeneous growth profiles inside gonococcal colonies, where growth rates dropped towards the COM of colonies. Our first assumption was that nutrient limitation causes heterogeneity in growth rates. To assess this hypothesis, we increased the flow rate 5 fold inside our flow chamber setup, but the improved nutrient supply did not prevent emergence of heterogeneous growth profiles. Besides mechanical constrains, limitations of oxygen or $\mathrm{CO}_{2}$ supply could influence bacterial growth dynamics. To test this assumption, 
$5 \mathrm{mM}$ sodium bicarbonate and $100 \mu \mathrm{M}$ sodium nitrite could be added to the growth medium. These supplements were added to the growth medium in previous experiments [166], but it was assumed that exclusion of this supplements does not effect growth of gonococcal colonies. However, spatio-temporal growth dynamics at cellular resolution could not have been analysed at this time. Consequently, it is likely that the previously mentioned supplements indeed influence gonococcal growth.

Next, we treated gonococcal colonies continuously with azithromycin during the whole experiment and obtained an early negative effect on growth rates. In future experiments it would be interesting to see how different concentrations of different antimicrobials effect bacterial spatio-temporal growth dynamics. Additionally, it would be interesting to measure how young freshly assembled bacterial colonies react to antimicrobial treatment in comparison to matured heterogeneous ones. To this end, we could start the treatment directly after injection of cells into our flow chamber setup or with a temporal shift to allow emergence of heterogeneous growth dynamics, which is the case after $2 \mathrm{~h}$ according to our results. Alternatively to antimicrobial treatment, DNA from commensal Neisseria strains was reported to act as a killing agent for pathogenic Neisseria strains [136]. Hereby, DNA with a foreign DNA methylation pattern needs to enter the pathogen and be recombined into its genome. Even though preliminary experiments indicate that Neisseria Elongata inhibits growth of $N$. gonorrhoeae and even leads to its extinction, these results could not have been properly evaluated and need to be repeated for this purpose.

In the course of this project, we aimed to find an alternative method to determine growth rates of spherical shaped bacteria that is independent of fluorescence labeling and confocal microscopy. For this purpose, Prof. Dr. Kohlheyer provided us with self-build microfluidic flow chambers. Microfluidic cultivation allows to study spatio-temporal growth dynamics at single cell resolution with bright-field microcopy. Growth rates of E.coli and C. glutamicum were successfully determined with this method [201]. One of his designs allowed us to grow two dimensional gonococcal colonies by placing separated containers with a limiting height of $1 \mu \mathrm{m}$ 
inside a flow channel. Even though we achieved in placing a few gonococci inside these growth containers, we observed that the flow channels were often blocked by strongly attached cells. In a few experiments, we managed to keep the flow channel cell-free while having a few cells inside growth containers. By manually counting the number of cells inside one growth container, we were able to estimate a growth rate by fitting an exponential function to the number of cells $N(t) / N_{0}=\exp (\lambda t)$. The estimated growth rate was $\lambda=0.32 \pm 0.01 h^{-1}$. In the future, clogging of the main channel of microfluidic flow chambers can be avoided by supplementing the growth medium for a short time period with water. 

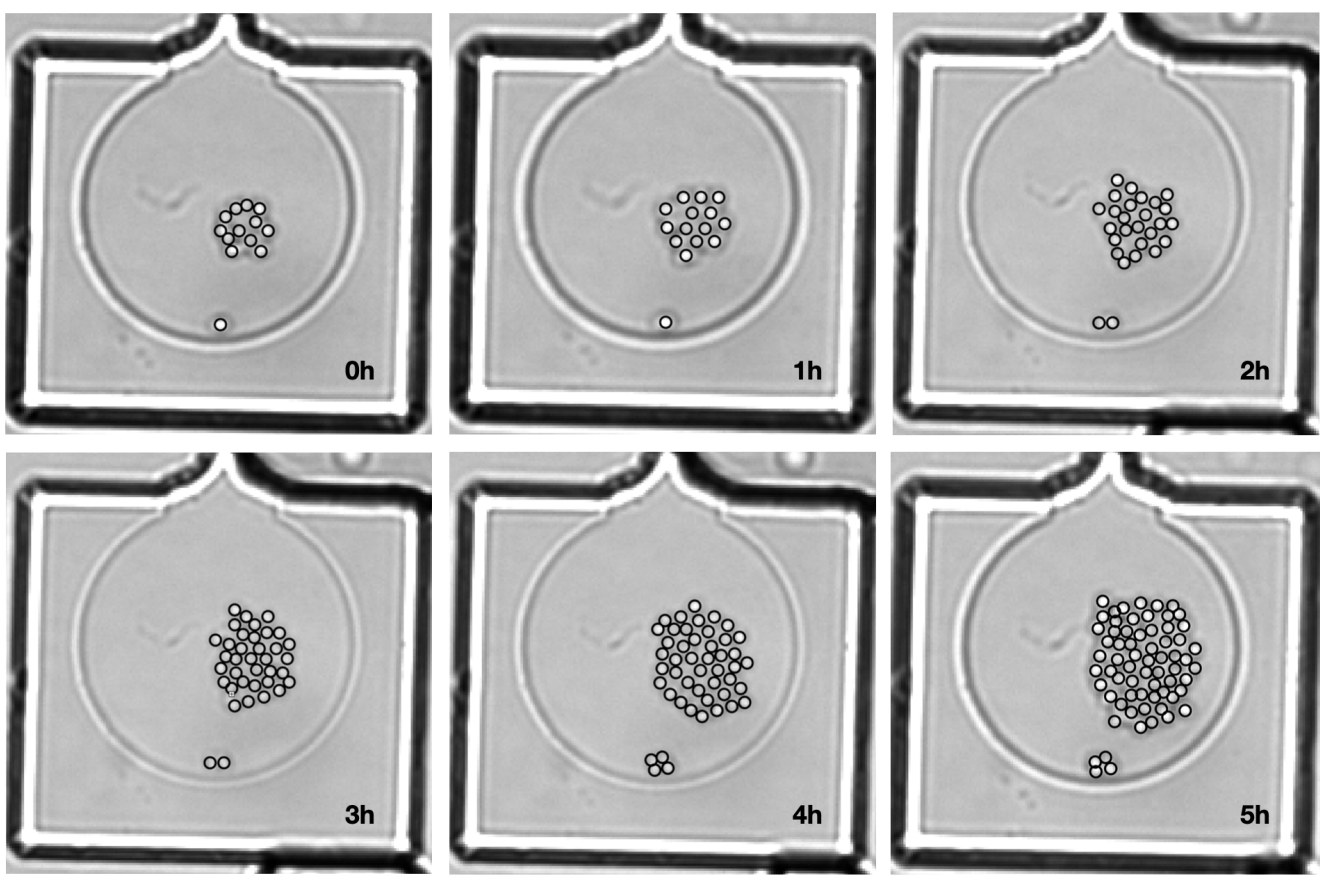

(a)

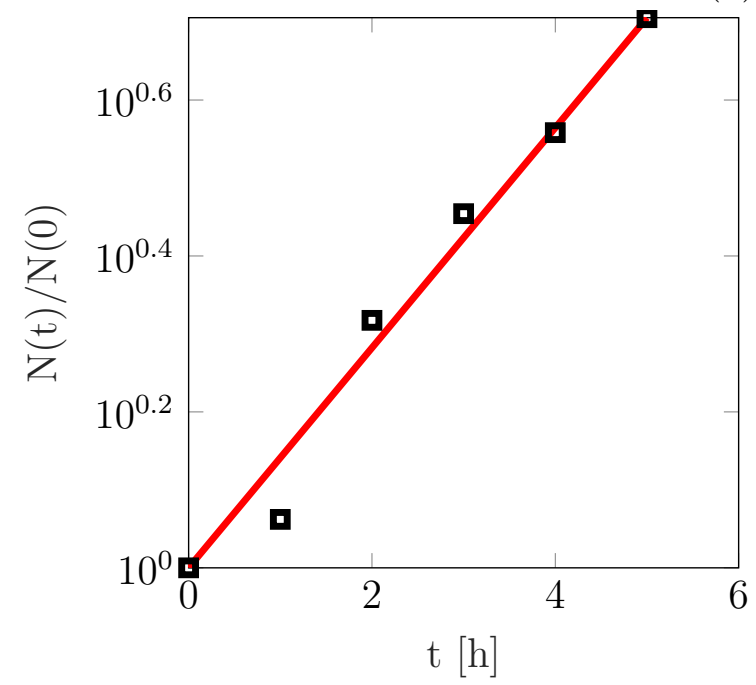

(b)

Figure 8.1: Growth of two dimensional gonococcal colonies. (a) Bright-field images of a growth container with a time resolution of 1 h. Circles mark show manually determined positions of cells. (b) Exponential function $N(t) / N_{0}=\exp (\lambda t)$ is fitted to the normalized number of cells $N(t) / N_{0}$ to estimate a growth rate $\lambda=0.32 \pm 0.01 h^{-1}$. 


\section{Appendix}

\subsection{Particle tracking software}

The particle tracking software is available as a MATLAB script. All image stacks should have a time resolution of $15 \mathrm{~min}$ and contain three channels, where the first channel should consist of a single bright-field image and the following two channels should be image stacks with an x-y-resolution of $80 \mathrm{~nm}$ and $400 \mathrm{~nm}$ in the z-direction.

After setting the path to the location of the nd2-file that contains all image stacks, the COM of colonies and their radii are determined from the bright-field images and saved as c.mat. The first two columns of $c$.mat contain the $\mathrm{x}-\mathrm{y}$-positions in pixels of the COM of colonies, the third column gives the radius of colonies in pixels, followed by time in frames and a parameter that describes the goodness of fitted cycles to the contours of colonies. Furthermore, three dimensional positions of cells are determined from the confocal image stacks of dead and living cells with sub-pixel resolution and saved as $r D$. mat and rL.mat. Both arrays contain seven columns with the following entries: $\mathrm{x}-, \mathrm{y}-$ and $\mathrm{z}$ - positions in $\mu \mathrm{m}$, the integrated brightness, squared radii of gyration and peak height of each cell, time in frames.

In the second part, the determined positions are linked into trajectories. At first, c.mat is corrected by comparing the radii and positions of successive frames. Then the height of the COM of colonies is estimated. This data is saved as COM.mat, which contains in its first columns the three dimensional positions of the COM of colonies in $\mu \mathrm{m}$ and in the last column the corresponding time. Using COM.mat, the positions of rL.mat and rD.mat are corrected in respect to the COM of colonies. Subsequently, the particle tracking algorithm is applied, giving dataCell.mat and 
dataDead.mat. dataCell.mat consists of $N$ columns, where $N$ depends on the total number of analysed clusters. In the first seven rows dataCell.mat contains the number of cells inside each cluster. The eighth row gives the time and the last two rows contain the distance of clusters to the edge of colonies and the radius of the colony. Additionally, a second array is included in dataCell.mat that gives information about cluster displacement relative to the COM of colonies. Here, the first seven entries in each column are replaced by the distance of each cluster to the colony COM in $\mu \mathrm{m}$. dataDead.mat is constructed differently. Each row contains the fraction of dead cells at different distances to the COM of colonies. The first entry of each row gives the fraction of dead cells at the COM of colonies with a binning of $2 \mu \mathrm{m}$. The next entry gives the fraction of dead cells $1 \mu \mathrm{m}$ away and so on. The last entry of each row contains the corresponding time. Finally, the MATLAB script computes growth rates from the radii of colonies, cell counting and velocity fields. 


\section{List of Figures}

3.1 Scheme of surface motility driven by T4P . . . . . . . . . . . . 9

3.2 Molecular model of T4P . . . . . . . . . . . . . . . . . . . . 10

3.3 Stages of biofilm development . . . . . . . . . . . . . . . 13

3.4 Repulsive and attractive interactions between bacteria . . . . . . 14

3.5 Biofilm structure of rod-shaped cells . . . . . . . . . . . . . 15

3.6 T4P-T4P interactions . . . . . . . . . . . . . . . . 16

3.7 Chemical heterogeneity in biofilms . . . . . . . . . . . . . . . . . . 19

5.1 Effect of a spatial bandpass filter on image stacks acquired with a

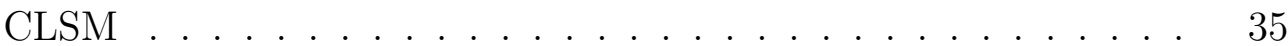

5.2 Image segmentation of image stacks acquired with a spinning disk confocal microscope . . . . . . . . . . . . . . . . . . . . . . . 36

5.3 Kernel used to filter confocal image stacks for single particle tracking 37

5.4 Effect of parameter variation for coordinate determination on RDF 41

5.5 Radius and COM detection from Bright-field images . . . . . . . . 43

5.6 Signal to noise ration for different confocal image slices . . . . . . 46

5.7 Examples of various exponential fits to the normalized number of cells 50

5.8 Illustration of distance to the edge or COM of colonies . . . . . . 51

5.9 Cell density profile through colony . . . . . . . . . . . . . . . 52

6.1 Liquid-like order of gonococcal $w t$ colonies . . . . . . . . . . 55

6.2 Sketch of how T4P interaction between adjacent cells mediate cell re-organization . . . . . . . . . . . . . . 56

6.3 Orthogonal views of image stacks of gonococcal colonies with different T4P motor properties . . . . . . . . . . . . . 57

6.4 Local cell density of gonococcal colonies . . . . . . . . . . . . 58 
6.5 Comparison of RDFs of colonies formed by strains with different T4P motor activity . . . . . . . . . . . . . . . . . 59

6.6 Remoteness distribution . . . . . . . . . . . . . . . . 61

6.7 Expression of $s f g f p$ reduces growth rate . . . . . . . . . . . 63

6.8 Lineage tracking in gonococcal colonies . . . . . . . . . . . . . . 64

6.9 Example of single growing cluster . . . . . . . . . . . . 66

6.10 Spatio-temporal dynamics of growth obtained by CC method . . . 67

6.11 Histogram of growth rates obtained from single clusters . . . . . . 69

6.12 Trajectories of individual clusters . . . . . . . . . . . . . 70

6.13 Growth rate determined from velocity field of clusters (VF method) 71

6.14 Effect of nutrients supply on growth rates . . . . . . . . . . . 73

6.15 Effect of nutrients supply on bacterial death . . . . . . . . . 74

6.16 Effect of stringent response on growth rates . . . . . . . . . . 75

6.17 Effect of stringent response on bacterial death . . . . . . . . 76

6.18 Effect of azithromycin treatment on growth rates . . . . . . . . 78

6.19 Effect of azithromycin treatment on bacterial death . . . . . . . 79

8.1 Growth of two dimensional gonococcal colonies . . . . . . . . . . . 91 


\section{List of Tables}

5.1 Ingredients for GC-Agar . . . . . . . . . . . . . . . . . 27

5.2 Ingredients for GC-Medium . . . . . . . . . . . . . . . . . . . . . . . . . . . . . . .

5.3 Ingredients for Isovitalex . . . . . . . . . . . . . . . . 28

5.4 List of used strains in this study . . . . . . . . . . . . . . . . 30

5.5 Ingredients for Mowiol . . . . . . . . . . . . . . . . . . 33 


\section{Bibliography}

[1] Berenike Maier. "How Physical Interactions Shape Bacterial Biofilms". In: Annual Review of Biophysics (2021). ISSN: 1936-122X. DOI: 10.1146/ annurev-biophys-062920-063646.

[2] J. W. Costerton et al. Bacterial biofilms in nature and disease. 1987. DoI: 10.1146/annurev.mi.41.100187.002251.

[3] J. W. Costerton. "Overview of microbial biofilms". In: Journal of Industrial Microbiology (1995). ISSN: 01694146. DOI: 10.1007/BF01569816.

[4] C. A. Fux et al. Survival strategies of infectious biofilms. 2005. DOI: 10 . 1016/j.tim.2004.11.010.

[5] Tom Cronenberg et al. "Antibiotics modulate attractive interactions in bacterial colonies affecting survivability under combined treatment". In: PLOS Pathogens (2021). DOI: 10.1371/journal.ppat. 1009251.

[6] Lucia Vidakovic et al. "Dynamic biofilm architecture confers individual and collective mechanisms of viral protection". In: Nature Microbiology (2017). ISSN: 20585276. DOI: 10.1038/s41564-017-0050-1.

[7] Hans Curt Flemming et al. Biofilms: An emergent form of bacterial life. 2016. DOI: $10.1038 /$ nrmicro.2016.94.

[8] Clayton W. Hall and Thien Fah Mah. Molecular mechanisms of biofilmbased antibiotic resistance and tolerance in pathogenic bacteria. 2017. DOI: 10.1093/femsre/fux010.

[9] Allan Konopka. "What is microbial community ecology". In: ISME Journal (2009). ISSN: 17517362. DOI: 10.1038/ismej .2009.88. 
[10] Sudani Abdul Rani et al. "Spatial patterns of DNA replication, protein synthesis, and oxygen concentration within bacterial biofilms reveal diverse physiological states". In: Journal of Bacteriology (2007). ISSN: 00219193. DOI: $10.1128 / \mathrm{JB} .00107-07$.

[11] Elizabeth J. Stewart et al. "Role of environmental and antibiotic stress on Staphylococcus epidermidis biofilm microstructure". In: Langmuir (2013). ISSN: 07437463. DOI: 10.1021/la401322k.

[12] Raimo Hartmann et al. Emergence of three-dimensional order and structure in growing biofilms. 2019. DOI: 10.1038/s41567-018-0356-9.

[13] Philip Pearce et al. "Flow-Induced Symmetry Breaking in Growing Bacterial Biofilms". In: Physical Review Letters (2019). ISSN: 10797114. DOI: 10.1103/ PhysRevLett. 123.258101.

[14] E. M. Purcell. "Life at low Reynolds number". In: American Journal of Physics (1977). ISSN: 0002-9505. DOI: 10.1119/1.10903.

[15] Howard C. Berg and Douglas A. Brown. "Chemotaxis in Escherichia coli analysed by three-dimensional tracking". In: Nature (1972). ISSN: 00280836. DOI: $10.1038 / 239500 a 0$.

[16] Steven H. Larsen et al. "Change in direction of flagellar rotation is the basis of the chemotactic response in Escherichia coli". In: Nature (1974). ISSN: 00280836. DOI: $10.1038 / 249074 \mathrm{a}$.

[17] Hans Curt Flemming and Jost Wingender. The biofilm matrix. 2010. DOI: $10.1038 /$ nrmicro2415.

[18] Robert Belas. Biofilms, flagella, and mechanosensing of surfaces by bacteria. 2014. DOI: $10.1016 /$ j.tim.2014.05.002.

[19] Pushkar P. Lele, Basarab G. Hosu, and Howard C. Berg. "Dynamics of mechanosensing in the bacterial flagellar motor". In: Proceedings of the National Academy of Sciences of the United States of America (2013). ISSN: 00278424. DOI: 10.1073/pnas. 1305885110.

[20] Berenike Maier and Gerard C.L. Wong. How Bacteria Use Type IV Pili Machinery on Surfaces. 2015. DOI: 10.1016/j.tim.2015.09.002. 
[21] Jeffrey M. Skerker and Howard C. Berg. "Direct observation of extension and retraction of type IV pili". In: Proceedings of the National Academy of Sciences of the United States of America (2001). ISSN: 00278424. DOI: 10.1073/pnas. 121171698.

[22] Lorenzo Talà et al. Pseudomonas aeruginosa orchestrates twitching motility by sequential control of type IV pili movements. 2019. DOI: 10.1038/s41564019-0378-9.

[23] Alexandre Persat et al. "Type IV pili mechanochemically regulate virulence factors in Pseudomonas aeruginosa". In: Proceedings of the National Academy of Sciences of the United States of America (2015). ISSN: 10916490. DOI: $10.1073 /$ pnas. 1502025112 .

[24] Courtney K. Ellison et al. "Obstruction of pilus retraction stimulates bacterial surface sensing". In: Science (2017). ISSN: 10959203. DOI: 10.1126/ science . aan5706.

[25] Rhett A. Snyder et al. "Surface sensing stimulates cellular differentiation in Caulobacter crescentus". In: Proceedings of the National Academy of Sciences of the United States of America (2020). ISSN: 10916490. DOI: 10.1073/pnas. 1920291117.

[26] Diane Bodenmiller, Evelyn Toh, and Yves V. Brun. "Development of Surface Adhesion in Caulobacter crescentus". In: Journal of Bacteriology (2004). ISSN: 00219193. DOI: 10.1128/JB.186.5.1438-1447. 2004.

[27] Alexey J. Merz, Magdalene So, and Michael P. Sheetz. "Pilus retraction powers bacterial twitching motility". In: Nature (2000). ISSN: 00280836. DOI: $10.1038 / 35024105$.

[28] Maxsim L. Gibiansky et al. Bacteria use type IV pili to walk upright and detach from surfaces. 2010. DOI: 10.1126/science.1194238.

[29] Jacinta C. Conrad et al. "Flagella and pili-mediated near-surface single-cell motility mechanisms in P. aeruginosa". In: Biophysical Journal (2011). ISSN: 15420086. DOI: $10.1016 /$ j .bpj . 2011 .02 .020. 
[30] Jens Eriksson et al. "Characterization of motility and piliation in pathogenic Neisseria”. In: BMC Microbiology (2015). DOI: 10.1186/s12866-015-04246.

[31] Claudia Holz et al. "Multiple pilus motors cooperate for persistent bacterial movement in two dimensions". In: Physical Review Letters (2010). ISSN: 10797114. DOI: 10.1103/PhysRevLett.104.178104.

[32] Daniel Wall and Dale Kaiser. Type IV pili and cell motility. 1999. DOI: 10.1046/j.1365-2958.1999.01339.x.

[33] Courtney K. Ellison et al. "Retraction of DNA-bound type IV competence pili initiates DNA uptake during natural transformation in Vibrio cholerae". In: Nature Microbiology (2018). ISSN: 20585276. DOI: $10.1038 /$ s41564018-0174-y.

[34] Cynthia D. Long et al. "Low-Level Pilin Expression Allows for Substantial DNA Transformation Competence in Neisseria gonorrhoeae". In: Infection and Immunity (2003). ISSN: 00199567. DOI: 10.1128/IAI .71.11.62796291.2003.

[35] David Bieber et al. Type IV pili, transient bacterial aggregates, and virulence of enteropathogenic escherichia coli. 1998. DOI: 10.1126/science. 280 . 5372.2114 .

[36] Mikkel Klausen et al. "Involvement of bacterial migration in the development of complex multicellular structures in Pseudomonas aeruginosa biofilms". In: Molecular Microbiology (2003). ISSN: 0950382X. DOI: 10.1046/j.13652958.2003.03677.x.

[37] Enno R. Oldewurtel et al. "Differential interaction forces govern bacterial sorting in early biofilms". In: eLife (2015). ISSN: 2050084X. DOI: 10.7554/ eLife.10811.

[38] Lisa Craig, Michael E. Pique, and John A. Tainer. Type IV pilus structure and bacterial pathogenicity. 2004. DOI: 10.1038/nrmicro885.

[39] Melissa Ayers, P. Lynne Howell, and Lori L. Burrows. Architecture of the type II secretion and type IV pilus machineries. 2010. DOI: 10.2217/fmb.10.76. 
[40] Lisa Craig, Katrina T. Forest, and Berenike Maier. Type IV pili: dynamics, biophysics and functional consequences. 2019. DOI: 10.1038/s41579-0190195-4.

[41] Lisa Craig and Juliana Li. Type IV pili: paradoxes in form and function. 2008. DOI: $10.1016 /$ j.sbi.2007.12.009.

[42] Yi Wei Chang et al. "Architecture of the type IVa pilus machine". In: Science (2016). ISSN: 10959203. DOI: 10.1126/science. aad2001.

[43] Kenneth A. Satyshur et al. "Crystal Structures of the Pilus Retraction Motor PilT Suggest Large Domain Movements and Subunit Cooperation Drive Motility". In: Structure (2007). ISSN: 09692126. DOI: 10.1016/j .str . 2007.01 .018$.

[44] Anton Welker et al. "Molecular Motors Govern Liquidlike Ordering and Fusion Dynamics of Bacterial Colonies". In: Physical Review Letters (2018). ISSN: 10797114. DOI: 10.1103/PhysRevLett.121.118102.

[45] Martin Clausen et al. "High-force generation is a conserved property of type IV pilus systems". In: Journal of Bacteriology (2009). ISSN: 00219193. DOI: $10.1128 / \mathrm{JB} .00396-09$.

[46] Berenike Maier et al. "Single pilus motor forces exceed $100 \mathrm{pN}$ ". In: Proceedings of the National Academy of Sciences of the United States of America (2002). ISSN: 00278424. DOI: 10.1073/pnas. 242523299.

[47] Dixon Ng et al. "The Vibrio cholerae Minor Pilin TcpB Initiates Assembly and Retraction of the Toxin-Coregulated Pilus". In: PLoS Pathogens (2016). ISSN: 15537374. DOI: 10.1371/journal.ppat.1006109.

[48] Jan Ribbe et al. "Role of cyclic di-GMP and exopolysaccharide in type IV pilus dynamics". In: Journal of Bacteriology (2017). ISSN: 10985530. DOI: 10.1128/JB.00859-16.

[49] Ana M. Misic, Kenneth A. Satyshur, and Katrina T. Forest. "P. aeruginosa PilT Structures with and without Nucleotide Reveal a Dynamic Type IV Pilus Retraction Motor". In: Journal of Molecular Biology (2010). ISSN: 00222836. DOI: $10.1016 / \mathrm{j} . j \mathrm{mb} .2010 .05 .066$. 
[50] Daria Bonazzi et al. "Intermittent Pili-Mediated Forces Fluidize Neisseria meningitidis Aggregates Promoting Vascular Colonization". In: Cell (2018). ISSN: 10974172. DOI: 10.1016/j.cell .2018.04.010.

[51] Robert Zöllner et al. "Type IV Pilin Post-Translational Modifications Modulate Material Properties of Bacterial Colonies". In: Biophysical Journal (2019). ISSN: 15420086. DOI: 10.1016/j.bpj .2019.01.020.

[52] Rainer Kurre and Berenike Maier. "Oxygen depletion triggers switching between discrete speed modes of gonococcal type IV pili". In: Biophysical Journal (2012). ISSN: 00063495. DOI: 10.1016/j.bpj .2012.04.020.

[53] Rainer Kurre et al. "Speed Switching of Gonococcal Surface Motility Correlates with Proton Motive Force". In: PLoS ONE (2013). ISSN: 19326203. DOI: $10.1371 /$ journal .pone. 0067718 .

[54] Michael R.W. Brown, David G. Allison, and Peter Gilbert. "Resistance of bacterial biofilms to antibiotics a growth-rate related effect?" In: Journal of Antimicrobial Chemotherapy (1988). ISSN: 03057453. DOI: 10.1093/jac/ 22.6 .777 .

[55] Luanne Hall-Stoodley, J. William Costerton, and Paul Stoodley. Bacterial biofilms: From the natural environment to infectious diseases. 2004. DOI: 10.1038/nrmicro821.

[56] Veysel Berk et al. "Molecular Architecture and Assembly Principles of Vibrio Cholerae Biofilms". In: Biophysical Journal (2012). ISSN: 00063495. DOI: $10.1016 / \mathrm{j} . \mathrm{bpj} .2011 .11 .3364$.

[57] Su Chuen Chew et al. "Dynamic remodeling of microbial biofilms by functionally distinct exopolysaccharides". In: mBio (2014). ISSN: 21507511. DOI: 10.1128/mBio.01536-14.

[58] Anna Dragoš et al. "Division of Labor during Biofilm Matrix Production". In: Current Biology (2018). ISSN: 09609822. DOI: 10.1016/j . cub. 2018.04.046.

[59] Karin Sauer et al. "Pseudomonas aeruginosa displays multiple phenotypes during development as a biofilm". In: Journal of Bacteriology (2002). ISSN: 00219193. DOI: 10.1128/jb.184.4.1140-1154.2002. 
[60] Russell D. Monds and George A. O'Toole. The developmental model of microbial biofilms: ten years of a paradigm up for review. 2009. DOI: 10 . 1016/j.tim.2008.11.001.

[61] J. H. Pringle and M. Fletcher. "Influence of substratum hydration and adsorbed macromolecules on bacterial attachment to surfaces". In: Applied and Environmental Microbiology (1986). ISSN: 00992240. DOI: 10.1128/aem. 51.6.1321-1325.1986.

[62] G. O'Toole, H. B. Kaplan, and R. Kolter. Biofilm formation as microbial development. 2000. DOI: 10.1146/annurev.micro.54.1.49.

[63] Knut Drescher et al. "Architectural transitions in Vibrio cholerae biofilms at single-cell resolution". In: Proceedings of the National Academy of Sciences of the United States of America (2016). ISSN: 10916490. DOI: 10.1073/pnas. 1601702113.

[64] Jeffrey B. Kaplan et al. "Low levels of $\beta$-Lactam antibiotics induce extracellular DNA release and biofilm formation in Staphylococcus aureus". In: mBio (2012). ISSN: 21507511. DOI: 10.1128/mBio.00198-12.

[65] Wook Kim et al. "Importance of positioning for microbial evolution". In: Proceedings of the National Academy of Sciences of the United States of America (2014). ISSN: 10916490. DOI: 10.1073/pnas.1323632111.

[66] Carsten Matz et al. "Microcolonies, quorum sensing and cytotoxicity determine the survival of Pseudomonas aeruginosa biofilms exposed to protozoan grazing". In: Environmental Microbiology (2004). ISSN: 14622912. DOI: $10.1111 / j .1462-2920.2004 .00556 . x$.

[67] Susanne Müller et al. "Predation by Myxococcus xanthus induces Bacillus subtilis to form spore-filled megastructures". In: Applied and Environmental Microbiology (2015). ISSN: 10985336. DOI: 10.1128/AEM. 02448-14.

[68] Xin Wang, Howard A. Stone, and Ramin Golestanian. "Shape of the growing front of biofilms". In: New Journal of Physics (2017). ISSN: 13672630. DOI: 10.1088/1367-2630/aa983f. arXiv: 1706.02372. 
[69] D. Dell'Arciprete et al. "A growing bacterial colony in two dimensions as an active nematic". In: Nature Communications (2018). ISSN: 20411723. DOI: $10.1038 / \mathrm{s} 41467-018-06370-3$.

[70] Zhihong You et al. "Geometry and Mechanics of Microdomains in Growing Bacterial Colonies". In: Physical Review X (2018). ISSN: 21603308. DOI: 10.1103/PhysRevX.8.031065. arXiv: 1703.04504.

[71] Farzan Beroz et al. "Verticalization of bacterial biofilms". In: Nature Physics (2018). ISSN: 17452481. DOI: 10.1038/s41567-018-0170-4. arXiv: 1801. 03064.

[72] Jiunn Cn Fong et al. "Structural dynamics of RbmA governs plasticity of Vibrio cholerae biofilms". In: eLife (2017). ISSN: 2050084X. DOI: 10.7554/ eLife. 26163.

[73] Manuel Maestre-Reyna, Wen Jin Wu, and Andrew H.J. Wang. "Structural insights into RbmA, a biofilm scaffolding protein of V. cholerae". In: PLoS ONE (2013). ISSN: 19326203. DOI: 10.1371/journal.pone.0082458.

[74] Jiunn C.N. Fong et al. "Identification and characterization of RbmA, a novel protein required for the development of rugose colony morphology and biofilm structure in Vibrio cholerae". In: Journal of Bacteriology (2006). ISSN: 00219193. DOI: 10.1128/JB.188 .3.1049-1059. 2006.

[75] Wolfram Pönisch et al. "Multiscale modeling of bacterial colonies: How pili mediate the dynamics of single cells and cellular aggregates". In: New Journal of Physics (2017). ISSN: 13672630. DOI: 10 . 1088/1367-2630/ aa5483.

[76] Lena Dewenter, Thorsten E. Volkmann, and Berenike Maier. "Oxygen governs gonococcal microcolony stability by enhancing the interaction force between type IV pili". In: Integrative Biology (United Kingdom) (2015). ISSN: 17579708. DOI: 10.1039/c5ib00018a.

[77] Nuno M. Oliveira et al. Correction: Biofilm Formation As a Response to Ecological Competition (PLoS Biol, 2015). 2015. DOI: 10.1371/journal. pbio.1002232. 
[78] Janus A.J. Haagensen et al. "Differentiation and distribution of colistin- and sodium dodecyl sulfate-tolerant cells in Pseudomonas aeruginosa biofilms". In: Journal of Bacteriology (2007). ISSN: 00219193. DOI: 10.1128/JB.0072006.

[79] Sünje Johanna Pamp et al. "Tolerance to the antimicrobial peptide colistin in Pseudomonas aeruginosa biofilms is linked to metabolically active cells, and depends on the pmr and mexAB-oprM genes". In: Molecular Microbiology (2008). ISSN: 0950382X. DOI: 10.1111/j.1365-2958.2008.06152.x.

[80] Thomas Bjarnsholt et al. "Pseudomonas aeruginosa tolerance to tobramycin, hydrogen peroxide and polymorphonuclear leukocytes is quorum-sensing dependent". In: Microbiology (2005). ISSN: 13500872. DOI: 10.1099/mic.0. 27463-0.

[81] Ehud Banin, Keith M. Brady, and E. Peter Greenberg. "Chelator-induced dispersal and killing of Pseudomonas aeruginosa cells in a biofilm". In: Applied and Environmental Microbiology (2006). ISSN: 00992240. DOI: 10. 1128/AEM . 72 . 3.2064-2069. 2006.

[82] Terry Brissac et al. "The meningococcal minor pilin PilX is responsible for type IV pilus conformational changes associated with signaling to endothelial cells". In: Infection and Immunity (2012). ISSN: 00199567. DOI: 10.1128/ IAI. 00369-12.

[83] Annegret Wilde and Conrad W. Mullineaux. Light-controlled motility in prokaryotes and the problem of directional light perception. 2017. DOI: 10 . 1093/femsre/fux045.

[84] Daniel K.H. Rode, Praveen K. Singh, and Knut Drescher. Multicellular and unicellular responses of microbial biofilms to stress. 2020. DOI: 10.1515/hsz2020-0213.

[85] Alexandre Persat et al. The mechanical world of bacteria. 2015. DOI: 10 . 1016/j. cell.2015.05.005.

[86] Dirk de Beer et al. "Effects of biofilm structures on oxygen distribution and mass transport". In: Biotechnology and Bioengineering (1994). ISSN: 10970290. DOI: 10.1002/bit. 260431118. 
[87] Jeff N. Anderl et al. "Role of nutrient limitation and stationary-phase existence in Klebsiella pneumoniae biofilm resistance to ampicillin and ciprofloxacin". In: Antimicrobial Agents and Chemotherapy (2003). ISSN: 00664804. DOI: 10.1128/AAC .47.4.1251-1256.2003.

[88] Marshall C. Walters et al. "Contributions of antibiotic penetration, oxygen limitation, and low metabolic activity to tolerance of Pseudomonas aeruginosa biofilms to ciprofloxacin and tobramycin". In: Antimicrobial Agents and Chemotherapy (2003). ISSN: 00664804. DOI: 10.1128/AAC.47.1.317323.2003.

[89] Giorgia Borriello et al. "Oxygen limitation contributes to antibiotic tolerance of Pseudomonas aeruginosa in biofilms". In: Antimicrobial Agents and Chemotherapy (2004). ISSN: 00664804. DOI: 10.1128/AAC .48 .7.26592664.2004.

[90] Erin Werner et al. "Stratified growth in Pseudomonas aeruginosa biofilms". In: Applied and Environmental Microbiology (2004). ISSN: 00992240. DOI: 10.1128/AEM. 70.10.6188-6196.2004.

[91] Philip S. Stewart et al. "Reaction-diffusion theory explains hypoxia and heterogeneous growth within microbial biofilms associated with chronic infections". In: npj Biofilms and Microbiomes (2016). ISSN: 20555008. DOI: 10.1038/npjbiofilms. 2016.12.

[92] E. Tuomanen et al. "The rate of killing of Escherichia coli by $\beta$-lactam antibiotics is strictly proportional to the rate of bacterial growth". In: Journal of General Microbiology (1986). ISSN: 00221287. DOI: 10.1099/ 00221287-132-5-1297.

[93] R. H.K. Eng et al. "Bactericidal effects of antibiotics on slowly growing and nongrowing bacteria". In: Antimicrobial Agents and Chemotherapy (1991). ISSN: 00664804. DOI: 10.1128/AAC .35.9.1824.

[94] D. J. Evans et al. "Susceptibility of pseudomonas aeruginosa and escherichia coli biofilms towards ciprofloxacin: Effect of specific growth rate". In: Journal of Antimicrobial Chemotherapy (1991). ISSN: 03057453. DOI: 10.1093/jac/ 27.2 .177 . 
[95] Z. Zheng and P. S. Stewart. "Growth limitation of Staphylococcus epidermidis in biofilms contributes to rifampin tolerance". In: Biofilms (2004). ISSN: $1479-0505$. DOI: $10.1017 / \mathrm{s} 1479050503001042$.

[96] Philip S. Stewart and Michael J. Franklin. Physiological heterogeneity in biofilms. 2008. DOI: 10.1038/nrmicro1838.

[97] Laurent A. Lardon et al. "iDynoMiCS: Next-generation individual-based modelling of biofilms". In: Environmental Microbiology (2011). ISSN: 14622912. DOI: $10.1111 / \mathrm{j} .1462-2920.2011 .02414 . \mathrm{x}$.

[98] John A. Cole et al. "Spatially-resolved metabolic cooperativity within dense bacterial colonies". In: BMC Systems Biology (2015). ISSN: 17520509. DOI: 10.1186/s12918-015-0155-1.

[99] Alma Dal Co, Simon Van Vliet, and Martin Ackermann. "Emergent microscale gradients give rise to metabolic cross-feeding and antibiotic tolerance in clonal bacterial populations". In: Philosophical Transactions of the Royal Society B: Biological Sciences (2019). ISSN: 14712970. DOI: 10.1098/rstb.2019.0080.

[100] Arthur Prindle et al. "Ion channels enable electrical communication in bacterial communities". In: Nature (2015). ISSN: 14764687. DOI: 10.1038/ nature15709.

[101] Jintao Liu et al. "Metabolic co-dependence gives rise to collective oscillations within biofilms". In: Nature (2015). ISSN: 14764687. DOI: 10.1038/ nature14660.

[102] Erin S. Gloag et al. "Self-organization of bacterial biofilms is facilitated by extracellular DNA". In: Proceedings of the National Academy of Sciences of the United States of America (2013). ISSN: 00278424. DOI: 10.1073/pnas. 1218898110.

[103] Matthew Ghannoum, Mahmoud Parsek, Marvin Whiteley, and Pranab K. Mukherjee. MICROBIAL BIOFILMS. 2015, p. 404. 
[104] Shuwen An, Ji'en Wu, and Lian Hui Zhang. "Modulation of pseudomonas aeruginosa biofilm dispersal by a cyclic-di-gmp phosphodiesterase with a putative hypoxia-sensing domain". In: Applied and Environmental Microbiology (2010). ISSN: 00992240. DOI: 10.1128/AEM. 01233-10.

[105] Tran T. Huynh et al. "Glucose starvation-induced dispersal of pseudomonas aeruginosa biofilms is camp and energy dependent". In: PLoS ONE (2012). ISSN: 19326203. DOI: 10.1371/journal.pone.0042874.

[106] Olga E. Petrova and Karin Sauer. Escaping the biofilm in more than one way: Desorption, detachment or dispersion. 2016. DOI: 10.1016/j.mib. 2016.01.004.

[107] Nicolas Barraud et al. "Involvement of nitric oxide in biofilm dispersal of Pseudomonas aeruginosa”. In: Journal of Bacteriology (2006). ISSN: 00219193. DOI: $10.1128 / \mathrm{JB} .00779-06$.

[108] Jessica L. Lister and Alexander R. Horswill. "Staphylococcus aureus biofilms: Recent developments in biofilm dispersal". In: Frontiers in Cellular and Infection Microbiology (2014). ISSN: 22352988. DOI: 10.3389/fcimb. 2014. 00178.

[109] Blaise R. Boles and Alexander R. Horswill. "agr-mediated dispersal of Staphylococcus aureus biofilms". In: PLoS Pathogens (2008). ISSN: 15537366. DOI: $10.1371 /$ journal.ppat. 1000052 .

[110] Praveen K. Singh et al. "Vibrio cholerae Combines Individual and Collective Sensing to Trigger Biofilm Dispersal". In: Current Biology (2017). ISSN: 09609822. DOI: $10.1016 /$ j.cub. 2017.09.041.

[111] Vasili Hauryliuk et al. Recent functional insights into the role of (p)ppGpp in bacterial physiology. 2015. DOI: 10.1038/nrmicro3448.

[112] Juana María Navarro Llorens, Antonio Tormo, and Esteban Martínez-García. Stationary phase in gram-negative bacteria. 2010. DOI: 10.1111/j.15746976.2010.00213.x.

[113] Lisa U. Magnusson, Anne Farewell, and Thomas Nyström. ppGpp: A global regulator in Escherichia coli. 2005. DOI: 10.1016/j.tim.2005.03.008. 
[114] Ian Booth. "Escherichia coli and Salmonella typhimurium; Cellular and Molecular Biology, Vol. 1 (of2)". In: Trends in Biochemical Sciences (1988). ISSN: 09680004. DOI: 10.1016/0968-0004 (88)90241-1.

[115] Anaïs Soares, Kévin Alexandre, and Manuel Etienne. Tolerance and Persistence of Pseudomonas aeruginosa in Biofilms Exposed to Antibiotics: Molecular Mechanisms, Antibiotic Strategies and Therapeutic Perspectives. 2020. DOI: $10.3389 / \mathrm{fmicb} .2020 .02057$.

[116] Dao Nguyen et al. "Active starvation responses mediate antibiotic tolerance in biofilms and nutrient-limited bacteria". In: Science (2011). ISSN: 10959203. DOI: $10.1126 /$ science. 1211037 .

[117] Oscar G. Gomez-Duarte et al. "Genetic diversity of the gene cluster encoding Longus, a type IV pilus of enterotoxigenic Escherichia coli". In: Journal of Bacteriology (2007). ISSN: 00219193. DOI: 10.1128/JB.00722-07.

[118] Paul A. Manning. "The tcp gene cluster of Vibrio cholerae". In: Gene. 1997. DOI: $10.1016 / \mathrm{S} 0378-1119(97) 00036-\mathrm{X}$.

[119] Kimberly K. Jefferson, Donald A. Goldmann, and Gerald B. Pier. "Use of confocal microscopy to analyze the rate of vancomycin penetration through Staphylococcus aureus biofilms". In: Antimicrobial Agents and Chemotherapy (2005). ISSN: 00664804. DOI: 10.1128/AAC . 49 .6 .24672473.2005.

[120] Rachna Singh et al. "Penetration barrier contributes to bacterial biofilmassociated resistance against only select antibiotics, and exhibits genus-, strain- and antibiotic-specific differences". In: Pathogens and Disease (2016). ISSN: 2049632X. DOI: 10.1093/femspd/ftw056.

[121] Rachna Singh et al. "Penetration of antibiotics through Staphylococcus aureus and Staphylococcus epidermidis biofilms". In: Journal of Antimicrobial Chemotherapy (2010). ISSN: 03057453. DOI: 10.1093/jac/dkq257.

[122] W. M. Dunne, E. O. Mason, and S. L. Kaplan. "Diffusion of rifampin and vancomycin through a Staphylococcus epidermidis biofilm". In: Antimicrobial Agents and Chemotherapy (1993). ISSN: 00664804. DOI: 10.1128/AAC. 37.12 .2522 . 
[123] Zhilan Zheng and Philip S. Stewart. "Penetration of rifampin through Staphylococcus epidermidis biofilms". In: Antimicrobial Agents and Chemotherapy (2002). ISSN: 00664804. DOI: 10.1128/AAC.46 .3.900-903.2002.

[124] Philip S. Stewart, William M. Davison, and Judith N. Steenbergen. "Daptomycin rapidly penetrates a Staphylococcus epidermidis biofilm". In: Antimicrobial Agents and Chemotherapy (2009). ISSN: 00664804. DOI: 10.1128/ AAC . 01728-08.

[125] Richard F. Collins et al. "Three-dimensional structure of the Neisseria meningitidis secretin PilQ determined from negative-stain transmission electron microscopy". In: Journal of Bacteriology (2003). ISSN: 00219193. DOI: $10.1128 / J B .185 .8 .2611-2617.2003$.

[126] Rym Boudjemaa et al. "New insight into daptomycin bioavailability and localization in Staphylococcus aureus biofilms by dynamic fluorescence imaging". In: Antimicrobial Agents and Chemotherapy (2016). ISSN: 10986596. DOI: $10.1128 / \mathrm{AAC} .00735-16$.

[127] G. Stone et al. "Tetracycline rapidly reaches all the constituent cells of uropathogenic Escherichia coli biofilms". In: Antimicrobial Agents and Chemotherapy (2002). ISSN: 00664804. DOI: 10 . 1128/AAC . 46 . 8 . 24582461.2002.

[128] Boo Shan Tseng et al. "The extracellular matrix protects Pseudomonas aeruginosa biofilms by limiting the penetration of tobramycin". In: Environmental Microbiology (2013). ISSN: 14622912. DOI: 10 .1111/14622920.12155.

[129] Kam Cheong Wong et al. "Antibiotic use for Vibrio infections: important insights from surveillance data". In: BMC Infectious Diseases (2015). ISSN: 14712334. DOI: $10.1186 / \mathrm{s} 12879-015-0959-z$.

[130] David W. Adams and Jeff Errington. Bacterial cell division: Assembly, maintenance and disassembly of the $Z$ ring. 2009. DOI: 10.1038/nrmicro2198.

[131] Francisco Díaz-Pascual et al. Breakdown of Vibrio cholerae biofilm architecture induced by antibiotics disrupts community barrier function. 2019. DOI: 10. 1038/s41564-019-0579-2. 
[132] Tâm Mignot et al. "Evidence that focal adhesion complexes power bacterial gliding motility". In: Science (2007). ISSN: 00368075. DOI: 10.1126/science. 1137223.

[133] Helen Fifer et al. "Failure of Dual Antimicrobial Therapy in Treatment of Gonorrhea". In: New England Journal of Medicine (2016). ISSN: 0028-4793. DOI: $10.1056 /$ nejmc1512757.

[134] David W. Eyre et al. "Gonorrhoea treatment failure caused by a Neisseria gonorrhoeae strain with combined ceftriaxone and high-level azithromycin resistance, England, February 2018". In: Eurosurveillance (2018). ISSN: 15607917. DOI: 10.2807/1560-7917.ES.2018.23.27.1800323.

[135] Magnus Unemo et al. Sexually transmitted infections: challenges ahead. 2017. DOI: $10.1016 /$ S1473-3099 (17) 30310-9.

[136] Won Jong Kim et al. "Commensal Neisseria Kill Neisseria gonorrhoeae through a DNA-Dependent Mechanism". In: Cell Host and Microbe (2019). ISSN: 19346069. DOI: 10.1016/j.chom.2019.07.003.

[137] Philipp J. Keller et al. "Reconstruction of zebrafish early embryonic development by scanned light sheet microscopy". In: Science (2008). ISSN: 00368075. DOI: $10.1126 /$ science. 1162493.

[138] Raphael Hornung et al. "Quantitative modelling of nutrient-limited growth of bacterial colonies in microfluidic cultivation". In: Journal of the Royal Society Interface (2018). ISSN: 17425662. DOI: 10.1098/rsif .2017.0713. arXiv: 1802.05858.

[139] A. M. Alsayed et al. "Chemistry: Premelting at defects within bulk colloidal crystals". In: Science (2005). ISSN: 00368075. DOI: $10.1126 /$ science . 1112399.

[140] Volkert W.A. De Villeneuve et al. "Chemistry: Colloidal hard-sphere crystal growth frustrated by large spherical impurities". In: Science (2005). ISSN: 00368075. DOI: 10.1126/science.1113207.

[141] Eric R. Weeks et al. "Three-dimensional direct imaging of structural relaxation near the colloidal glass transition". In: Science (2000). ISSN: 00368075. DOI: $10.1126 /$ science. 287.5453 .627$. 
[142] John C. Crocker and David G. Grier. "Methods of digital video microscopy for colloidal studies". In: Journal of Colloid and Interface Science (1996). ISSN: 00219797. DOI: 10.1006/jcis.1996.0217.

[143] Yongxiang Gao and Maria L. Kilfoil. "Accurate detection and complete tracking of large populations of features in three dimensions". In: Optics Express (2009). ISSN: 1094-4087. DOI: 10.1364/oe.17.004685.

[144] Zoe N. Demou and Larry V. McIntire. "Fully automated three-dimensional tracking of cancer cells in collagen gels: Determination of motility phenotypes at the cellular level". In: Cancer Research (2002). ISSN: 00085472.

[145] Frank P. Ferrie, Martin D. Levine, and Steven W. Zucke. "Cell Tracking: A Modeling and Minimization Approach". In: IEEE Transactions on Pattern Analysis and Machine Intelligence (1982). ISSN: 01628828. DOI: 10.1109/ TPAMI . 1982.4767244.

[146] Martin D. Levine et al. "QUANTIFICATION OF BLOOD CELL MOTION BY A METHOD OF AUTOMATIC DIGITAL PICTURE PROCESSING." In: IEEE Transactions on Pattern Analysis and Machine Intelligence (1980). ISSN: 01628828. DOI: 10.1109/TPAMI. 1980.6592365.

[147] S. T. Young et al. Real-time tracing of spermatozoa. 1996. DOI: 10.1109/ 51.544519 .

[148] Luc Vincent, Luc Vincent, and Pierre Soille. "Watersheds in Digital Spaces: An Efficient Algorithm Based on Immersion Simulations". In: IEEE Transactions on Pattern Analysis and Machine Intelligence (1991). ISSN: 01628828. DOI: $10.1109 / 34.87344$.

[149] V. Grau et al. "Improved watershed transform for medical image segmentation using prior information". In: IEEE Transactions on Medical Imaging (2004). ISSN: 02780062. DOI: 10.1109/TMI .2004.824224.

[150] Hakan Ancin et al. "Advances in automated 3-D image analysis of cell populations imaged by confocal microscopy". In: Cytometry (1996). ISSN: 01964763. DOI: 10 . 1002/ (SICI) 1097-0320 (19961101) $25: 3<221:$ : AIDCYTO3>3.0.CO;2-I. 
[151] Christophe De Hauwer et al. "In vitro motility evaluation of aggregated cancer cells by means of automatic image processing". In: Cytometry (1999). ISSN: 01964763. DOI: 10 . 1002/(SICI ) 1097-0320(19990501) $36: 1<1:$ : AID-CYT01>3.0.CO;2-P.

[152] Gang Lin et al. "A Hybrid 3D Watershed Algorithm Incorporating Gradient Cues and Object Models for Automatic Segmentation of Nuclei in Confocal Image Stacks". In: Cytometry Part A (2003). ISSN: 15524922. DOI: 10.1002/ cyto.a. 10079.

[153] Norberto Malpica et al. "Applying watershed algorithms to the segmentation of clustered nuclei". In: Cytometry (1997). ISSN: 01964763. DoI: 10.1002/ (SICI) 1097-0320 (19970801) 28:4<289: : AID-CYT03>3. 0. CO; 2-7.

[154] Mathieu Leocmach and Hajime Tanaka. "A novel particle tracking method with individual particle size measurement and its application to ordering in glassy hard sphere colloids". In: Soft Matter (2013). ISSN: 1744683X. DOI: 10.1039/c2sm27107a.

[155] Philip Ball. "Colloids get active". In: Nature Materials (2013). ISSN: 14761122. DOI: $10.1038 / \mathrm{nmat3} 720$.

[156] Martin Vorregaard. "Comstat2 - a modern 3D image analysis environment for biofilms". PhD thesis. 2008.

[157] Raimo Hartmann et al. "Quantitative image analysis of microbial communities with BiofilmQ". In: Nature Microbiology (2021). ISSN: 20585276. DOI: $10.1038 / \mathrm{s} 41564-020-00817-4$.

[158] C. Potera. Studying slime. 1998. DOI: 10.1289/ehp.98106a604.

[159] C. Potera. Forging a link between biofilms and disease. 1999. DoI: 10.1126/ science.283.5409.1837.

[160] Xue Zhong et al. "Titanium surface priming with phase-transited lysozyme to establish a silver nanoparticle-loaded chitosan/hyaluronic acid antibacterial multilayer via layer-by-layer self-assembly". In: PLoS ONE (2016). ISSN: 19326203. DOI: 10.1371/journal .pone. 0146957. 
[161] Thomas Bjarnsholt et al. Applying insights from biofilm biology to drug development-can a new approach be developed? 2013. DOI: 10 .1038/ $\operatorname{nrd} 4000$.

[162] Morten Alhede et al. "Phenotypes of non-attached pseudomonas aeruginosa aggregates resemble surface attached biofilm". In: PLoS ONE (2011). ISSN: 19326203. DOI: 10.1371/journal.pone.0027943.

[163] Robert C. Shields et al. "Efficacy of a Marine Bacterial Nuclease against Biofilm Forming Microorganisms Isolated from Chronic Rhinosinusitis". In: PLoS ONE (2013). ISSN: 19326203. DOI: 10.1371/journal.pone.0055339.

[164] E. A. Izano et al. "Detachment and killing of Aggregatibacter actinomycetemcomitans biofilms by dispersin B and SDS". In: Journal of Dental Research (2007). ISSN: 00220345. DOI: 10.1177/154405910708600707.

[165] Steven A. Rogers et al. "Synergistic effects between conventional antibiotics and 2-aminoimidazole-derived antibiofilm agents". In: Antimicrobial Agents and Chemotherapy (2010). ISSN: 00664804. DOI: 10.1128/AAC.01418-09.

[166] Nadzeya Kouzel, Enno R. Oldewurtel, and Berenike Maier. "Gene transfer efficiency in gonococcal biofilms: Role of biofilm age, architecture, and pilin antigenic variation". In: Journal of Bacteriology (2015). ISSN: 10985530. DOI: $10.1128 / \mathrm{JB} .00171-15$.

[167] Ella Rotman and H. Steven Seifert. "The genetics of Neisseria species". In: Annual Review of Genetics (2014). ISSN: 15452948. DOI: 10.1146/annurevgenet-120213-092007.

[168] Rainer Kurre et al. "PilT2 enhances the speed of gonococcal type IV pilus retraction and of twitching motility". In: Molecular Microbiology (2012). ISSN: 0950382X. DOI: $10.1111 / \mathrm{mmi} .12022$.

[169] Laty A. Cahoon and H. Steven Seifert. "An alternative DNA structure is necessary for pilin antigenic variation in Neisseria gonorrhoeae". In: Science (2009). ISSN: 00368075. DOI: 10.1126/science.1175653. 
[170] Scott D. Fisher et al. "RelA alone appears essential for (p)ppGpp production when Neisseria gonorrhoeae encounters nutritional stress". In: FEMS Microbiology Letters (2005). ISSN: 03781097. DOI: 10.1016/j .femsle. 2005. 05.014.

[171] Robert Zöllner et al. "Phase and antigenic variation govern competition dynamics through positioning in bacterial colonies". In: Scientific Reports (2017). ISSN: 20452322. DOI: 10.1038/s41598-017-12472-7.

[172] Anton Welker et al. "Spatio-temporal dynamics of growth and death within spherical bacterial colonies". In: Biophys. J. in press (2021).

[173] Bente Børud et al. "Genetic, structural, and antigenic analyses of glycan diversity in the O-linked protein glycosylation systems of human Neisseria species". In: Journal of Bacteriology (2010). ISSN: 00219193. DOI: 10.1128/ JB. 00101-10.

[174] R. Scott, M. P. Allen, and D. J. Tildesley. "Computer Simulation of Liquids." In: Mathematics of Computation (1991). ISSN: 00255718. DOI: 10.2307/ 2938686.

[175] T. H. Besseling et al. "Determination of the positions and orientations of concentrated rod-like colloids from 3D microscopy data". In: Journal of Physics Condensed Matter. 2015. DoI: 10.1088/0953-8984/27/19/194109. arXiv: 1406.4985 .

[176] S. J. Hewlett and T. Wilson. "Resolution enhancement in three-dimensional confocal microscopy". In: Machine Vision and Applications (1991). ISSN: 09328092. DOI: $10.1007 /$ BF01815300.

[177] Karole L. Blythe, Eric J. Titus, and Katherine A. Willets. "ObjectiveInduced Point Spread Function Aberrations and Their Impact on SuperResolution Microscopy". In: Analytical Chemistry (2015). ISSN: 15206882. DOI: $10.1021 /$ acs . analchem. 5b01848.

[178] Brian C. Carter, George T. Shubeita, and Steven P. Gross. "Tracking single particles: A user-friendly quantitative evaluation". In: Physical Biology (2005). ISSN: 14783975. DOI: $10.1088 / 1478-3967 / 2 / 1 / 008$. 
[179] Michael K. Cheezum, William F. Walker, and William H. Guilford. "Quantitative comparison of algorithms for tracking single fluorescent particles". In: Biophysical Journal (2001). ISSN: 00063495. DOI: 10 . 1016/S00063495 (01) 75884-5.

[180] David J. Stephens and Victoria J. Allan. Light microscopy techniques for live cell imaging. 2003. DOI: 10.1126/science. 1082160.

[181] M. Bamdad et al. "A new expression for radial distribution function and infinite shear modulus of Lennard-Jones fluids". In: Chemical Physics (2006). ISSN: 03010104. DOI: 10.1016/j. chemphys.2006.02.001.

[182] Enrico Matteoli and G. Ali Mansoori. "A simple expression for radial distribution functions of pure fluids and mixtures". In: The Journal of Chemical Physics (1995). ISSN: 00219606. DOI: 10.1063/1.470654.

[183] J. Hendricks et al. "Different mechanisms for dynamical arrest in largely asymmetric binary mixtures". In: Physical Review E - Statistical, Nonlinear, and Soft Matter Physics (2015). IsSN: 15502376. DOI: 10.1103/PhysRevE. 91.032308 .

[184] Morgen E. Anyan et al. "Type IV pili interactions promote intercellular association and moderate swarming of Pseudomonas aeruginosa". In: Proceedings of the National Academy of Sciences of the United States of America (2014). ISSN: 10916490. DOI: 10.1073/pnas.1414661111.

[185] Alyson M. Hockenberry et al. "Attenuation of the type IV pilus retraction motor influences Neisseria gonorrhoeae social and infection behavior". In: mBio (2016). ISSN: 21507511. DOI: 10.1128/mBio.01994-16.

[186] Dustin L. Higashi et al. "Dynamics of Neisseria gonorrhoeae attachment: Microcolony development, cortical plaque formation, and cytoprotection". In: Infection and Immunity (2007). ISSN: 00199567. DOI: 10.1128/IAI.0068707.

[187] Bruno M. Saraiva et al. "Reassessment of the distinctive geometry of Staphylococcus aureus cell division". In: Nature Communications (2020). ISSN: 20411723. DOI: $10.1038 / \mathrm{s} 41467-020-17940-9$. 
[188] Garima Kapoor, Saurabh Saigal, and Ashok Elongavan. Action and resistance mechanisms of antibiotics: A guide for clinicians. 2017. DOI: 10 . 4103/joacp. JOACP_349_15.

[189] Paul L. Biancaniello, Anthony J. Kim, and John C. Crocker. "Colloidal interactions and self-assembly using DNA hybridization". In: Physical Review Letters (2005). ISSN: 00319007. DOI: 10.1103/PhysRevLett.94. 058302.

[190] Oya Ilke Sentürk et al. "Independent Blue and Red Light Triggered Narcissistic Self-Sorting Self-Assembly of Colloidal Particles". In: Small (2019). ISSN: 16136829. DOI: 10.1002/smll.201901801.

[191] Wolfram Pönisch et al. "Pili mediated intercellular forces shape heterogeneous bacterial microcolonies prior to multicellular differentiation". In: Scientific Reports (2018). ISSN: 20452322. DOI: $10.1038 /$ s41598-01834754-4.

[192] Kai Zhou et al. "Non-equilibrium Dynamics of Growing Bacterial Colonies". In: (2021).

[193] Manuela Dietrich et al. "Pilin regulation in the pilT mutant of Neisseria gonorrhoeae strain MS11". In: FEMS Microbiology Letters (2009). ISSN: 03781097. DOI: $10.1111 / \mathrm{j} .1574-6968.2009 .01647 . \mathrm{x}$.

[194] Robert Zöllner, Tom Cronenberg, and Berenike Maier. "Motor properties of pilt-independent type 4 pilus retraction in gonococci". In: Journal of Bacteriology. 2019. DOI: 10.1128/JB.00778-18.

[195] Etienne Carbonnelle et al. "A systematic genetic analysis in Neisseria meningitidis defines the Pil proteins required for assembly, functionality, stabilization and export of type IV pili". In: Molecular Microbiology (2006). ISSN: 0950382X. DOI: $10.1111 / j .1365-2958.2006 .05341 . x$.

[196] Mya R. Warren et al. "Spatiotemporal establishment of dense bacterial colonies growing on hard agar". In: eLife (2019). ISSN: 2050084X. DOI: 10.7554/eLife. 41093 .

[197] Morgan Delarue et al. "Self-driven jamming in growing microbial populations". In: Nature Physics (2016). ISSN: 17452481. DOI: 10.1038/nphys3741. 
[198] Hannah H. Tuson et al. "Measuring the stiffness of bacterial cells from growth rates in hydrogels of tunable elasticity". In: Molecular Microbiology (2012). ISSN: 0950382X. DOI: 10.1111/j.1365-2958.2012.08063.x.

[199] J. M. Andrews. "Determination of minimum inhibitory concentrations". In: Journal of Antimicrobial Chemotherapy (2001). ISSN: 03057453. DOI: 10.1093/jac/48.suppl_1.5.

[200] Joe J. Harrison et al. "Microtiter susceptibility testing of microbes growing on peg lids: A miniaturized biofilm model for high-throughput screening". In: Nature Protocols (2010). ISSN: 17502799. DOI: 10.1038/nprot.2010.71.

[201] A. Grünberger et al. "High-Throughput Growth Rate Determination of Bacteria Microcolonies on Single Cell Level". In: Chemie Ingenieur Technik (2012). DOI: $10.1002 /$ cite. 201250342. 


\section{Danksagung}

Hiermit möchte ich mich bei allen bedanken, die mich während dieser Arbeit begleitet und unterstützt haben.

An erster Stelle bedanke ich mich besonders bei Prof. Dr. Berenike Maier für die tatkräftige Unterstützung während meiner Projekte und die Möglichkeit mir diese frei auswählen du dürfen. Du hast dir für mich immer Zeit genommen, wenn ich Hilfe brauchte und hattest gute Ratschläge parat. Außerdem danke ich für die Möglichkeit meine Ergebnisse auf Konferenzen presäntieren zu dürfen.

Zusätzlich bedanke ich mich bei Prof. Dr. Tobias Bollenbach und Prof. Dr. Ines Neundorf für das Interesse an meiner Arbeit und die Teilnahme in meiner Prüfungskommission als zweiter Gutachter und als Vorsitzende.

Ich danke der AG Maier für das angenehme Arbeitsklima und die großartige Unterstützung während der Corona-Zeit. Ganz besonders bedanke ich mich bei Tom, Marc und Niklas für ihre Hilfsbereitschaft und Expertise. Weiterhin bedanke ich mich bei Thorsten Volkmann für seine brillianten technischen Ideen beim Bau von Inkubatoren.

Darüber hinaus bedanke ich mich bei Thorsten Auth für die Organisation und Koordination von Biosoft.

Zum Schluss bedanke ich mich bei meiner Familie für das zahlreiche Babysitten. Besonderer Dank geht an Ines, Finn und Lina, die immer für mich da sind. 


\section{Erklärung}

Hiermit versichere ich an Eides statt, dass ich die vorliegende Dissertation selbstständig und ohne die Benutzung anderer als der angegebenen Hilfsmittel und Literatur angefertigt habe. Alle Stellen, die wörtlich oder sinngemaäß aus veröffentlichten und nicht veroöffentlichten Werken dem Wortlaut oder dem Sinn nach entnommen wurden, sind als solche kenntlich gemacht. Ich versichere an Eides statt, dass diese Dissertation noch keiner anderen Fakultaät oder Universität zur Prüfung vorgelegen hat; dass sie - abgesehen von unten angegebenen Teilpublikationen und eingebundenen Artikeln und Manuskripten - noch nicht veröffentlicht worden ist sowie, dass ich eine Veröffentlichung der Dissertation vor Abschluss der Promotion nicht ohne Genehmigung des Promotionsausschusses vornehmen werde. Die Bestimmungen dieser Ordnung sind mir bekannt. Darüber hinaus erkläre ich hiermit, dass ich die Ordnung zur Sicherung guter wissenschaftlicher Praxis und zum Umgang mit wissenschaftlichem Fehlverhalten der Universität zu Köln gelesen und sie bei der Durchführung der Dissertation zugrundeliegenden Arbeiten und der schriftlich verfassten Dissertation beachtet habe und verpflichte mich hiermit, die dort genannten Vorgaben bei allen wissenschaftlichen Tätigkeiten zu beachten und umzusetzen. Ich versichere, dass die eingereichte elektronische Fassung der eingereichten Druckfassung vollständig entspricht.

\section{Teilpublikationen:}

- Welker, A.* , Cronenberg, T.*, Zöllner, R.*, Meel, C., Siewering, K., Bender, N., Hennes, M., Oldewurtel, E.R., Maier, B., Molecular motors govern liquidlike ordering and fusion dynamics of bacterial colonies, Phys. Rev. Lett., 121, 118102 (2018) 
- Zöllner, R.*, Cronenberg, T.*, Kouzel, N., Welker, A., Koomey, M., Maier, B., Type IV pilin post-translational modifications modulate materials properties of bacterial colonies, Biophys. J., 116(5), 938 (2019)

- Welker, A.*, Hennes, M,* Bender, N., Cronenberg, T., Schneider, G., Maier, B., Spatio-temporal dynamics of growth and death within spherical bacterial colonies, Biophys. J. in press (2021)

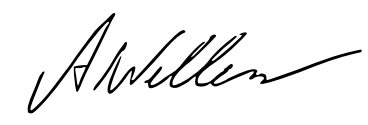

Köln, 03.09.2021

Anton Welker 